\title{
On finite-temperature holographic QCD in the Veneziano limit
}

\author{
T. Alho, ${ }^{a}$ M. Järvinen, ${ }^{b}$ K. Kajantie, ${ }^{c}$ E. Kiritsis ${ }^{b, d, 1}$ and K. Tuominen ${ }^{a, c}$
}

${ }^{a}$ Department of Physics, P.O. Box 35, FI-40014 University of Jyväskylä, Finland ${ }^{2}$

${ }^{b}$ Crete Center for Theoretical Physics, Department of Physics, University of Crete, P.O. Box 2208, 71003 Heraklion, Greece ${ }^{3}$

${ }^{c}$ Helsinki Institute of Physics, P.O. Box 64, FI-00014 University of Helsinki, Finland ${ }^{4}$

${ }^{d}$ APC, Université Paris 7, CNRS/IN2P3 (UMR du CNRS 7164), CEA/IRFU, Obs. de Paris, Sorbonne Paris, Cité, Bâtiment Condorcet, F-75205, Paris Cedex 13, France ${ }^{5}$

E-mail: timo.s.alho@jyu.fi, mjarvine@physics.uoc.gr, keijo.kajantie@helsinki.fi, kimmo.i.tuominen@jyu.fi

ABstRact: Holographic models in the $T=0$ universality class of QCD in the limit of large number $N_{c}$ of colors and $N_{f}$ massless fermion flavors, but constant ratio $x_{f}=N_{f} / N_{c}$, are analyzed at finite temperature. The models contain a 5-dimensional metric and two scalars, a dilaton sourcing $\operatorname{Tr} F^{2}$ and a tachyon dual to $\bar{q} q$. The phase structure on the $T, x_{f}$ plane is computed and various 1st order, 2nd order transitions and crossovers with their chiral symmetry properties are identified. For each $x_{f}$, the temperature dependence of $p / T^{4}$ and the condensate $\langle\bar{q} q\rangle$ is computed. In the simplest case, we find that for $x_{f}$ up to the critical $x_{c} \sim 4$ there is a 1st order transition on which chiral symmetry is broken and the energy density jumps. In the conformal window $x_{c}<x_{f}<11 / 2$, there is only a continuous crossover between two conformal phases. When approaching $x_{c}$ from below, $x_{f} \rightarrow x_{c}$, temperature scales approach zero as specified by Miransky scaling.

KEYWORDS: Gauge-gravity correspondence, AdS-CFT Correspondence

ARXIV EPRINT: 1210.4516

\footnotetext{
${ }^{1}$ Contact information can be found here: http://hep.physics.uoc.gr/ ${ }^{\sim}$ kiritsis/

${ }^{2}$ https://www.jyu.fi/fysiikka/en

${ }^{3}$ http://hep.physics.uoc.gr

${ }^{4}$ http://www.hip.fi

${ }^{5}$ http://www.apc.univ-paris7.fr
} 


\section{Contents}

1 Introduction 1

1.1 V-QCD at zero temperature 3

$\begin{array}{lll}1.2 & \text { Black holes without tachyon hair } & 7\end{array}$

$\begin{array}{lll}1.3 & \text { Black holes with tachyon hair and zero quark mass } & 8\end{array}$

$\begin{array}{lll}1.4 & \text { The phase structure of different V-QCD models } & 9\end{array}$

2 Defining V-QCD $\quad 11$

2.1 Gravity action of the model 11

$\begin{array}{ll}2.2 \text { Construction of the potentials } & 13\end{array}$

2.2.1 The potentials from the beta function in the UV 13

$\begin{array}{lll}2.2 .2 & \text { Large } \lambda \text { behavior of the potentials } & 15\end{array}$

$\begin{array}{lll}2.2 .3 & \text { IR fixed point and the BF bound for the tachyon } & 17\end{array}$

3 V-QCD at finite temperature: equations and their solution $\quad 19$

$\begin{array}{ll}3.1 \text { Equations and numerical solution } & 19\end{array}$

$\begin{array}{ll}3.2 & \text { Physical quantities } \\ 3.3 & \text { Fixing quark }\end{array}$

$\begin{array}{lll}3.3 & \text { Fixing quark mass } & 22\end{array}$

3.3.1 Zero quark mass 23

3.3.2 Nonzero quark mass 24

$\begin{array}{lll}3.4 & \text { Thermodynamics } & 25\end{array}$

4 Results for the phase structure $\quad 30$

$\begin{array}{ll}4.1 \text { Phase transitions } & 30\end{array}$

4.2 Class-II potentials 33

$\begin{array}{lll}4.3 & \text { Class-II } * \text { potentials } & 37\end{array}$

$\begin{array}{lll}4.4 & \text { Class-I potentials } & 38\end{array}$

$\begin{array}{lll}4.5 & \text { Class- } I_{*} \text { potentials } & 39\end{array}$

$\begin{array}{lll}\text { 4.6 PotI with logarithmic correction to } \kappa(\lambda) & 39\end{array}$

$\begin{array}{lll}4.7 & \text { The conformal window } & 42\end{array}$

4.8 The limits $x_{f} \rightarrow 0$ and $x_{f} \rightarrow x_{c} \quad 43$

5 The chiral condensate 46

6 Nonzero quark mass and thermodynamics $\quad 48$

$\begin{array}{lll}7 & \text { Outlook } & 49\end{array}$

A UV expansions $\quad \mathbf{5 1}$

A.1 Fields $\lambda$ and $A \quad 51$

A.2 The tachyon $\quad 52$

A.3 Finite temperature 53 
B IR expansions $\quad 54$

B.1 Zero temperature 54

B.1.1 $A$ and $\lambda \quad 54$

B.1.2 The tachyon $\quad 55$

B.2 Finite temperature 56

$\begin{array}{ll}\text { C The quark mass and the Efimov solutions } & 57\end{array}$

$\begin{array}{ll}\text { D Computation of } \lambda_{\text {end }} & 58\end{array}$

$\begin{array}{ll}\text { E Computation of the condensate } & 59\end{array}$

\section{Introduction}

QCD in the Veneziano limit [1],

$$
N_{c} \rightarrow \infty, \quad N_{f} \rightarrow \infty, \quad \frac{N_{f}}{N_{c}}=x_{f} \text { fixed, } \quad \lambda=g_{\mathrm{YM}}^{2} N_{c} \text { fixed }
$$

is expected to display a host of interesting and mostly non-perturbative phenomena, including:

- The "conformal window" with a nontrivial infrared (IR) fixed point, which extends from $x_{f}=\frac{11}{2}$ to smaller values of $x_{f}$. The region $x_{f} \rightarrow \frac{11}{2}$ has an IR fixed point while the theory is still weakly coupled, as was analyzed in [2] (see also [3, 4]).

- It is expected that at a critical $x_{f}=x_{c}$, the conformal window will end, and for $x_{f}<x_{c}$, the theory will exhibit chiral symmetry breaking in the IR. This behavior is expected to persist down to $x_{f}=0$. For $x_{f}>x_{c}$ the IR theory is a conformal field theory at strong coupling, that progressively becomes weak as $x_{f} \rightarrow \frac{11}{2}$.

- Near and below $x_{c}$, there is the transition region to conventional QCD IR behavior. In this region the theory is expected to be "walking": This means that the theory appears to be approaching the IR fixed point as the coupling evolves very slowly for many e-foldings of energies. But chiral symmetry breaking is nevertheless triggered and in the deep infrared the coupling diverges as in QCD. The slow evolution of the coupling has been correlated with a nontrivial dimension for the quark mass operator near two, rather than three (the free field value). IR observables are expected to obey the Miransky scaling [5-7] as $x_{f} \rightarrow x_{c}$ from below.

- New phenomena are expected to appear at finite density driven by strong coupling and the presence of quarks. These include color superconductivity $[8,9]$. In this case, however, gauge invariant vevs are effectively double trace operators and the phase structure is determined at the next to leading order in $1 / N_{c}$. 
The existence of the "walking" region makes the theory extremely interesting for applications in dynamical electroweak symmetry breaking (technicolor). This has also motivated an intensive lattice Monte Carlo work during recent years [10-14]. The bulk of this work has been done at zero temperature; recently there appeared the first attempts to go to finite $T$ for QCD with $N_{c}=3, N_{f}$ up to 8 [14-16] and for non-QCD-like theories [17]. Chiral effective theories have also been applied [18-25].

The aim of the present work is to study a class of holographic bottom-up models (VQCD) that belong to the universality class of QCD with massless quarks in the Veneziano limit [26] at finite temperature and zero chemical potential. We will calculate the temperature dependence of the free energy density $(=-$ pressure $=-p(T))$ and of the quark condensate $(\langle\bar{q} q\rangle(T))$. The former acts as an effective order parameter for deconfinement (at large $N_{c}$ ), for which there is no true order parameter associated with a symmetry. ${ }^{1}$ The quark condensate is a true order parameter for chiral symmetry if quarks are massless. The calculation is carried out for the full range of $x_{f}, 0<x_{f}<11 / 2$.

Discontinuities or rapid variations in pressure (or energy density) and quark condensate can be used to define phase boundaries associated with deconfinement and chiral symmetry restoration temperatures $T_{d}\left(x_{f}\right)$ and $T_{\chi}\left(x_{f}\right)$. We will use the usual nomenclature: If the $n$th derivative of $p(T)$ is discontinuous, the transition is of $n$th order. We also consider continuous crossovers which are identified by using the scaled quantity $(\epsilon-3 p) / T^{4}$. Its maximum defines the crossover temperature $T_{\text {crossover }}\left(x_{f}\right)$. The phase diagram is defined as a plot of all phase boundaries on the $\left(x_{f}, T\right)$ plane. The phase diagrams we present will also contain a rich structure of metastable states, namely local (but globally subleading) minima of the free energy.

In the holographic approach the thermal transitions will be transitions between various 5-dimensional black hole and "thermal gas" metrics and the nomenclature of transitions, explained later in great detail, will be correspondingly different. The holographic approach is constrained but not fully constrained and we cannot give a precise prediction of the phase diagram of hot $\mathrm{V}$-QCD. We can state the most plausible behavior but we can also mention a few other alternatives. We will always find the analogues of $T_{d}$ and $T_{\chi}$, but we will also find transitions with no obvious QCD interpretation. Whether these reflect real physics of hot QCD in the Veneziano limit or whether they are artifacts of the holographic approach will be an interesting problem for further study.

The usual expectation is that there is a 1 st order line at $T_{d}=T_{\chi}$; in the large $N_{c}$ limit one can actually prove that $T_{\chi} \geq T_{d}[18,19]$. The main class of our predictions reflect these properties: for smaller $x_{f}$ we find that deconfinement and chiral symmetry restoration coincide, but for $x_{f}$ approaching $x_{c}$ the deconfining and chiral transitions can become separate so that $T_{\chi}>T_{d}$ (see, for example, figure 13 below). The chiral transition is then of 2nd order (and mean field type). Furthermore, for smaller $x_{f}$ the separate 2nd order chiral transition is in the metastable region so that it can be reached if the system is supercooled [27]. One might here add that $T_{\chi}<T_{d}$ for stable phases may be reached at large chemical potential $[28,29]$.

\footnotetext{
${ }^{1} \mathrm{~A}$ related one, used commonly in lattice work, is the expectation value of the Polyakov loop.
} 
The starting point of our finite temperature analysis is the $T=0$ holographic model introduced in [26], based on previous theoretical ideas in [30-40]. Moving to finite $T$ implies studying black hole solutions of the action in [26]. A defining characteristic of this class of models is that they contain full backreaction between the duals of the color and flavor degrees of freedom. Earlier work [41-48] on thermodynamics in such bottom-up models imposed quasiconformality directly on the beta function of the theory. One should note that walking behavior and the related "conformal transition" at $x_{f}=x_{c}$ have also been studied in top-down models [49-54], as well as in simpler bottom-up models [55-60] which do not attempt to model the backreaction. See also [40,61] on introducing backreacted flavor in the top-down models.

In this introduction we will first describe the special properties of V-QCD from [26] and then discuss general properties of its black hole solutions. Section 2 will contain a detailed discussion of the action of the model and of the two characteristic classes of scalar potentials. Section 3 presents the Einstein equations of the model, describes how they are numerically solved and, finally, how thermodynamics is computed from the numerical bulk fields. A particularly delicate issue here is the fixing of the quark mass $m$ to zero. We also briefly comment on fixing $m$ to some nonzero value. An extensive list of numerical results is given in section 4. From these, the types of phase transition lines the models predict are determined. In section 5, techniques for computing the condensate are described and several numerical results are given. One should note that this, as well as many other numerical issues in the model, are technically very demanding. Finally, section 6 contains a discussion of what are the effects of making the quark mass nonzero. Several detailed considerations are collected in appendices.

\section{$1.1 \quad$ V-QCD at zero temperature}

In [26] a class of bottom-up holographic models was introduced (named V-QCD) and shown to be in the universality class of QCD in the Veneziano limit at zero temperature and density. These were 5-dimensional models of two scalars coupled to gravity. One of the scalars, the "dilaton" $\lambda$, is dual to $\operatorname{Tr}\left[F^{2}\right]$ (the QCD gauge coupling constant, or more precisely the 't Hooft coupling). The other scalar, the "tachyon" $\tau$, is dual to the quark mass operator $\bar{q} q$. The potentials and interactions were modeled along successful bottomup models for YM, namely Improved Holographic QCD (IHQCD) [30-36] and the idea that string theory tachyon condensation describes chiral symmetry breaking [37-39, 62-65].

The bulk action considered was

$$
S=S_{g}+S_{f}, \quad S_{g}=M^{3} N_{c}^{2} \int d^{5} x \sqrt{-g}\left[R-\frac{4}{3} \frac{(\partial \lambda)^{2}}{\lambda^{2}}+V_{g}(\lambda)\right],
$$

with $\lambda$ the 't Hooft coupling (exponential of the dilaton $\phi$ ) and the tachyon ${ }^{2}$ action $^{3}$

$$
S_{f}=-x_{f} M^{3} N_{c}^{2} \int d^{5} x V_{f}(\lambda, \tau) \sqrt{\operatorname{det}\left(g_{\mu \nu}+\kappa(\lambda) \partial_{\mu} \tau \partial_{\nu} \tau\right)} .
$$

\footnotetext{
${ }^{2}$ We have taken the tachyon to be real and diagonal in flavor space.

${ }^{3}$ To find the vacuum (saddle point) solution we have set the gauge fields $A_{\mu}^{L, R}$ dual to the QCD currents to zero, as they are not expected to have vacuum expectation values at zero density. We have also suppressed the Wess-Zumino terms as they also do not contribute to the vacuum solution.
} 
The pure glue potential $V_{g}$ has been determined from previous studies [33-35]. The tachyon potential $V_{f}(\lambda, \tau)$ must satisfy some basic properties determined by the dual theory or by general properties of tachyons in string theory: (a) To provide the proper dimension for the dual operator near the boundary (b) To exponentially vanish for $\tau \rightarrow \infty$. The function $\kappa(\lambda)$ captures, among other things, the transformation from the string frame to the Einstein frame in five dimensions. The class of potentials that were investigated in [26] are of the form

$$
V_{f}(\lambda, \tau)=V_{0 f}(\lambda) e^{-a(\lambda) \tau^{2}}
$$

In the Veneziano limit, the back-reaction of the flavor sector on the glue sector is fully included.

As with IHQCD, it was arranged that the theory is asymptotically AdS in the UV up to logarithmic corrections in the bulk coordinate. The function $V_{0 f}(\lambda)$ is such that the potential $V_{g}(\lambda)-x_{f} V_{0 f}(\lambda)$, when the tachyon has not condensed $(\tau=0)$ has an extremum ${ }^{4}$ at a finite value $\lambda=\lambda_{*}$. As we approach the Banks-Zaks region [2], $x_{f} \rightarrow \frac{11}{2}$, the value of $\lambda_{*}$ approaches zero. Without the tachyon, $\tau=0$, the equations of motion imply that also $\beta\left(\lambda_{*}\right)=0$, i.e., $\lambda_{*}$ is an IR fixed point. When the dynamics of $\tau$ is included, the system approaches $\lambda_{*}$ but is driven away from it as long as $x_{f}<x_{c}$ (see figure7 of [26]).

The dimension of the chiral condensate was calculated in the IR fixed point theory from the bulk equations. It was found that it decreases monotonically with $x_{f}$ for reasonably chosen potentials. It crossed the value 2 at $x_{f}=x_{c}$ where $x_{c}$ corresponds to the end of the conformal window as argued in [66].

The lower edge of the conformal window $x_{c}$ lies in the vicinity of 4 . Requiring the holographic $\beta$-functions to match with QCD in the UV, we find that

$$
3.7 \lesssim x_{c} \lesssim 4.2
$$

where the bounds are not strict but hold approximately for potentials that have smooth $\lambda$-dependence in the UV.

There is also a phenomenological heuristic argument for the value $x_{c} \approx 4$, simply from counting degrees of freedom. At low $T$ chiral symmetry is broken and the massless degrees of freedom are $N_{f}^{2}$ Goldstone bosons. At large $T$ there are $2 N_{c}^{2}+\frac{7}{2} N_{c} N_{f}$ weakly coupled degrees of freedom. These numbers are equal for $x_{f}=4$. Conformal window and the location of its edge was also discussed within holographic frameworks related to V-QCD in $[45,67-69]$.

Apart from $x_{f}$, there is a single parameter in the theory, namely $\frac{m}{\Lambda_{\mathrm{QCD}}}$ where $m$ is the UV value of the (flavor independent) quark mass. For each value of $x_{f}$, the bulk equations were solved with fixed sources corresponding to fixed $m, \Lambda_{\mathrm{QCD}}$. The vevs were determined such that the solution is "regular" in the IR. The notion of regularity is tricky even in the case of IHQCD (pure glue), as there is a naked singularity in the far IR. For the dilaton this has been settled in [30-35]. For the tachyon the notion of regularity is different and has been studied in detail in $[38,39]$.

\footnotetext{
${ }^{4}$ The extremum may exist for all $0<x_{f}<\frac{11}{2}$ or may disappear at some small $x_{f}$. No changes in the phase structure at zero temperature for these two cases were found in [26].
} 
The regularity condition was implemented in the IR. After solving the equations from the IR to the UV (this was done mostly numerically), there is a single parameter that determines the solutions as well as the UV coupling constants and vevs, and this is a real number $\tau_{0}$ controlling the value of the tachyon in the IR. This number reflects the single dimensionless parameter $\frac{m}{\Lambda_{\mathrm{QCD}}}$ of the theory.

For different values of $x_{f}$ and $m$ the following qualitatively different regions were found in $[26]$ :

- When $x_{c} \leq x_{f}<11 / 2$ and $m=0$, the theory flows to an IR fixed point. The IR conformal field theory is weakly coupled near $x_{f}=\frac{11}{2}$ and strongly coupled in the vicinity of $x_{c}$. Chiral symmetry is unbroken in this regime (this is known as the conformal window).

- When $x_{c} \leq x_{f}<11 / 2$ and $m \neq 0$, the tachyon has a nontrivial profile, and there is a single solution with the given source, which is "regular" in the IR. The IR theory is a theory with a mass gap.

- When $0<x_{f}<x_{c}$ and $m=0$, there is an infinite number of regular solutions with nontrivial tachyon profile, and a special solution with an identically vanishing tachyon and a nontrivial IR fixed point. The infinite number of solutions with nontrivial tachyon are classified by their number of zeros. The solution with the lowest free energy is the one with no zeros.

- When $0<x_{f}<x_{c}$ and $m \neq 0$, the theory has vacua with nontrivial profile for the tachyon. For every non-zero $m$, there is a finite number of regular solutions that grows as $m$ approaches zero.

In [26] two large classes of tachyon potentials were identified. Potentials in class I, have $a(\lambda)$ constant in (1.4). In this case the tachyon diverges exponentially in the IR for the regular solution

$$
\tau \underset{r \rightarrow \infty}{\sim} \tau_{0} \exp [C r],
$$

where $C$ is a known constant (see appendix B) and $\tau_{0}$ is the only integration constant controlling the solution. It determines the source (mass) in the UV. Potentials in class II, have $a(\lambda) \sim \lambda^{\frac{2}{3}}$ as $\lambda \rightarrow \infty$, and a tachyon that diverges in a milder way in the IR as

$$
\tau \underset{r \rightarrow \infty}{\sim} C \sqrt{r-r_{1}}
$$

where again $C$ is known and $r_{1}$ is the single integration constant controlling the regular solution. The qualitative conclusions above and below were valid for both classes of potentials.

In the region $x_{f}<x_{c}$ where several solutions exist, there is a interesting relation between the IR value $\tau_{0}$ controlling the regular solutions, and the UV parameters, namely $m$. This is determined numerically, and a relevant plot describing the relation between $m$ and $\tau_{0}$ at fixed $x_{f}$ is in figure 1.

The solutions are characterized by the number of times $n$ the tachyon field changes sign as it evolves from the UV to the IR. For all values of $m$ there is a single solution with 
no tachyon zeroes. In addition, for each positive $n$ there are two solutions ${ }^{5}$ which exist within a finite range $0<m<m_{n}$, where the limiting value $m_{n}$ decreases with increasing $n$, and one solution for $m=0$. In particular, for large enough fixed $m$, we find that only the solution without tachyon zeroes exists.

For $m \neq 0$, out of all regular solutions, the "first" one without tachyon zeroes has the smallest free energy. The same is true for $m=0$, namely the solution with nontrivial tachyon without zeroes is energetically favored over the solutions with positive $n$ as well as over the special solution with identically vanishing tachyon, which appears only for $m=0$ and would leave chiral symmetry unbroken. Therefore, chiral symmetry is broken for $x_{f}<x_{c}$.

In the region just below $x_{c},[26]$ found Miransky scaling for the chiral condensate. As $x_{f} \rightarrow x_{c}$,

$$
\sigma=\langle\bar{q} q\rangle \sim \Lambda_{\mathrm{QCD}}^{3} \exp \left(-\frac{2 \hat{K}}{\sqrt{x_{c}-x_{f}}}\right) .
$$

For $x \geq x_{c}$, let $m_{\mathrm{IR}}(x)$ be the mass of the tachyon at the IR fixed point and $\ell_{\mathrm{IR}}(x)$ the IR AdS radius. The coefficient $\hat{K}$ is then fixed as

$$
\hat{K}=\frac{\pi}{\sqrt{\frac{d}{d x}\left[m_{\mathrm{IR}}^{2} \ell_{\mathrm{IR}}^{2}\right]_{x=x_{c}}}} .
$$

The behavior at and below the conformal transition at $x_{f}=x_{c}$ is to a large extent independent of the details of the model. In particular, no information on the nonlinear terms in the tachyon EoM is needed or how the IR boundary conditions are fixed. In the same region, "walking" of gauge coupling is realized. The YM coupling flows from small values to values very near $\lambda_{*}$, remains approximately constant for many e-foldings of energy (in this regime the tachyon remains small), and then runs off to infinity, driven by a large value of the tachyon field in the IR. The walking is related to a long section of the solution which is similar to the one studied in earlier bottom-up models for walking [55-59].

The finite temperature analysis of V-QCD amounts to studying all black hole solutions with appropriate boundary conditions. To start with, any zero temperature solution becomes a candidate saddle point at finite temperature by compactifying time on a circle of radius $\beta$. Any other competing black hole solution must have the same boundary conditions as well as a regular horizon in the IR.

As the dilaton always has a nontrivial UV source, it will always have a nontrivial profile in the black-hole solutions. With the tachyon, things can be different. In the massless case, its source is zero. Therefore there are two possible options (as in the zero temperature configurations discussed above): either it is identically zero (if the vev $\langle\bar{q} q\rangle$ is also zero) or it is non-zero (implying a non-zero vev).

Therefore we have two large classes of black holes in the massless case: those with $\tau=0$ and those with $\tau \neq 0$. We will first consider the tachyon-free class.

\footnotetext{
${ }^{5}$ As $m$ and $-m$ are related by a chiral rotation by $\pi$, we can take $m \geq 0$.
} 


\subsection{Black holes without tachyon hair}

If $\tau=0$, we have black holes in a single scalar theory, with potential $V(\lambda)=V_{g}(\lambda)-$ $x_{f} V_{0 f}(\lambda)$ from (1.4). This is a potential with an extremum for $x_{f} \neq 0^{6}$ and no extremum when $x_{f}=0$.

Black hole solutions for such potentials were discussed in generality in [33-35]. After fixing all invariances, they are characterised by a single IR constant, $\lambda_{h}$, the value of the dilaton at the horizon. The plot relating the temperature $T$ to $\lambda_{h}$ contains important information about the thermodynamics of such black holes. Small values of $\lambda_{h}$ denote large black-holes whereas larger values of $\lambda_{h}$ correspond to smaller black holes (smaller horizon size and entropy). In all plots of this paper, dilatonic black holes without tachyon hair are denoted by red lines in the respective $\left(\lambda_{h}, T\right)$-diagrams, and we shall call the corresponding function $T_{u}\left(\lambda_{h}\right)$.

When $x_{f}=0, \lambda$ can become arbitrarily large at zero temperature, implying that $\lambda_{h}$ can also be arbitrarily large for the finite temperature configurations. At finite temperature there are two branches: large black holes which are stable and small black holes which are unstable. If $T_{u}^{\prime}\left(\lambda_{h}\right)<0$ the black-hole thermodynamics is stable, otherwise it is unstable. There is a minimum temperature above which such black holes exist as shown, for example, by the black line in figure 22 (left or right).

When $x_{f}>0$, we have two possibilities. The first is that the potential $V_{\text {eff }}(\lambda)=$ $V_{g}(\lambda)-x_{f} V_{0 f}(\lambda)$ has an extremum at $\lambda \rightarrow \lambda_{*}(x)$ for all $0<x_{f}<\frac{11}{2}$, with $\lambda_{*}\left(x_{f} \rightarrow \frac{11}{2}\right) \rightarrow 0$ and $\lambda_{*}\left(x_{f} \rightarrow 0\right) \rightarrow \infty$. The second is that such extremum only exists for $x>x_{*}$, where $x_{*}<x_{c}$. We shall denote these potentials with a star subscript.

At finite temperature, and when the potential $V_{\text {eff }}$ has no extremum, the black hole without the tachyon hair exists for all positive $\lambda_{h}$. For the potentials studied here, function $T_{u}\left(\lambda_{h}\right)$ is qualitatively similar to the YM case $\left(x_{f} \rightarrow 0\right)$ [33-35]. As shown in figure 17 (top-left) and in figure 19 (left), there are two black hole branches, which exist above some minimum temperature. The branch at low $\lambda_{h}$ is thermodynamically stable, while the large- $\lambda_{h}$ branch is unstable.

When the extremum is present, $0<\lambda_{h}<\lambda_{*}(x)$. The temperature of the black-hole corresponding to $\lambda_{h}=\lambda_{*}(x)$ is $T=0$, while that of $\lambda_{h} \rightarrow 0$ has $T \rightarrow \infty$. There is no minimum temperature here. For any temperature there is always at least one black-hole solution. There are several possibilities that are shown as red lines in figures 7 (left), 9 (top), 10 (left) and 12 (left).

When $x_{f}$ is large, but still smaller than $x_{c}$, the $T=T_{u}\left(\lambda_{h}\right)$ relation is one-to-one but contains a bump (a change of concavity) like in figure 9 (top). Then this is accompanied by a crossover behavior, signaled by a bump in the trace of the stress tensor $(\epsilon-3 p) / T^{4}$, (aka interaction measure) as shown in figure 9 (bottom-right).

At low enough $x_{f}$, the relation $T=T_{u}\left(\lambda_{h}\right)$ is not always one-to-one, as can be seen in figure 10 (left) or in figure 22. Then there are points where $T_{u}^{\prime}\left(\lambda_{h}\right)=0$. In such a case there can be a first order transition between the stable branches of the black hole solutions. This is a remnant of the deconfining transition at $x_{f}=0$ (pure YM). In figure 22 both left and

\footnotetext{
${ }^{6}$ The extremum may also exist only for $x_{f}$ above some fixed $x_{*}$, see the discussion further below.
} 


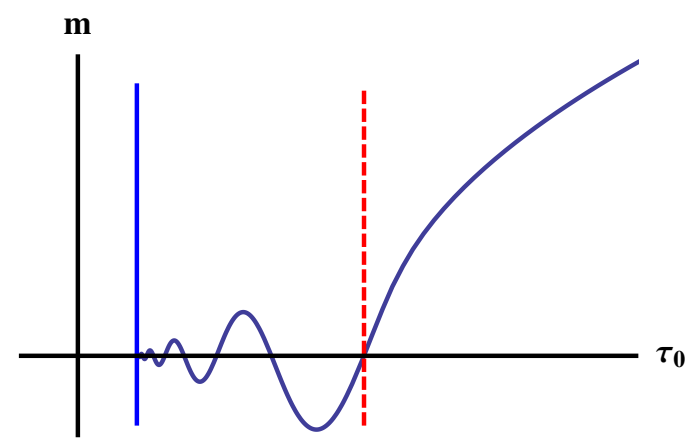

Figure 1. Plot of the UV Mass parameter $m$, as a function of the IR scale $\tau_{0}$ in (1.6), for $x_{f}<x_{c}$. The vertical solid blue line marks the end-point of the existence of regular solutions. The dashed red line indicates the appearance of more than one regular solution with the same value of $m$.

right several curves in the $\left(T, \lambda_{h}\right)$-plane for different $x_{f}$ indicate the successive structure of dilaton black holes (red lines). The black line corresponds to the pure YM $\left(x_{f}=0\right)$ limit.

When $x>x_{c}$ we are in the conformal window. The only black holes that exist here are those without tachyon hair. The relation $T=T_{u}\left(\lambda_{h}\right)$ is monotonic and there is a continuous transition to the black-hole phase at $T=0^{+}$, as in the AdS case in the Poincaré patch. The thermodynamic functions, especially the interaction measure, show a crossover maximum at a temperature that is moving towards the UV as $x_{f} \rightarrow \frac{11}{2}$.

\subsection{Black holes with tachyon hair and zero quark mass}

When $\tau \neq 0$ we have black holes in the two scalar theory. The tachyon starts as $\sim r^{3}$ near the UV boundary as the source (quark mass) vanishes. In all plots of this paper, such black holes (with both dilaton and tachyon hair) are denoted by blue lines in the respective $\left(\lambda_{h}, T\right)$-diagrams, and we shall denote the corresponding functions by $T_{b}\left(\lambda_{h}\right)$. They are still one parameter solutions and can be parametrized again by the value $\lambda_{h}$ of $\lambda$ at the horizon, which translates into the temperature. These black holes usually exist for all $\left.x_{f} \in\right] 0, x_{c}$ [ and our discussion below focuses in this region.

Because the presence of the nontrivial tachyon perturbs and annuls the possible nontrivial IR fixed point, for such black-holes, $\lambda_{h}$ can take arbitrarily large values. This can be seen from the blue lines in figures 7 (left), 9 (top), 10 (left) and 12 (left). For all such black holes, the chiral condensate is determined by the regularity of the black hole solution. It decreases as $\lambda_{h}$ decreases, and at some point it vanishes. At this point, the blue line in the $\left(\lambda_{h}, T\right)$-diagram merges with the red line corresponding to a $\lambda_{h}$ that we call $\lambda_{\text {end }}$ throughout the paper. This can be seen in all the figures mentioned above.

The shape of the blue line can vary as a function of $x_{f}$ and the type of potential. There are three typical examples of shapes:

- Simple lines that are monotonic as the one depicted in figure 12 (left). This is an example of a monotonic blue branch where all such black-holes are thermodynamically unstable. Moreover, they have a minimum temperature. In such a case, they can never be thermodynamically dominant. At some temperature the vacuum thermal 
solution is dominated by a dilaton black hole on the red line, and the chiral restoration transition is 1 st order.

- Lines with two branches as the one depicted in figure 10 (left). Here the blue line has two parts one (to the left) that is thermodynamically stable and another to the right that is thermodynamically unstable. In such a case, the system is in the thermal vacuum solution at low enough temperatures, then jumps with a 1st order transition to the tachyon-hairy solution (the part of the blue line that is thick in figure 10 (left)) which still break chiral symmetry, and then eventually smoothly transits to the red line at the point where the blue and red lines merge, via a chirally-restoring 2 nd order transition. ${ }^{7}$

- Lines with more than two branches as the one depicted in figure 11 (left). In this example the blue line has four branches, two unstable and two stable. There are in total three phase transitions here, first from the vacuum thermal solution to the rightmost blue thick branch, then to the intermediate thick blue branch and finally a 2nd order chirally restoring transition to the red branch at the point they touch. In this case there are two 1st order transitions between three chirally breaking phases, and a 2 nd order one to the chirally symmetric phase.

A concrete overall view of the $x_{f}$ dependence is presented in figure 2 , in which $T\left(\lambda_{h}\right)$ is plotted for potentials of type II with SB normalisation (definitions specified later) for various $x_{f}$. One sees clearly how the pure (black) YM curve is approached for $x_{f} \rightarrow 0$. The thick curves represent stable phases; when a thick curve ends, the system makes a 1st order transition to the low $T$ phase. When thick curves change from red to blue curves, a 2nd order transition to a chirally broken phase takes place. For a more accurate picture of small $x_{f}$, see figure 22 .

\subsection{The phase structure of different V-QCD models}

There are three main ingredients that characterize a priori different QCD models which, however, have the same phase structure and qualitative behavior at zero temperature:

- The asymptotics of the tachyon solution in the IR. This is controlled by the behavior of the function $a(\lambda)$ in the tachyon potential in (1.4). When $a(\lambda)$ is constant, the tachyon diverges exponentially in the IR, and we call such potentials of type I. When $a(\lambda)$ diverges as $\lambda^{\frac{2}{3}}$ in the IR ( $\lambda$ large) then the tachyon diverges as a square root in the IR, and we call such potentials of type II.

- For any potential, the UV constant factor $W_{0}$ of $V_{0 f}(\lambda)$ in (1.4), defined in (2.13) can vary in finite range, which in appropriate units is $\left.] 0, \frac{24}{11}\right]$, as in $(2.21)$. We pick for each type of potential three indicative values of $\mathcal{L}_{0}^{2} W_{0}$ that in general might

\footnotetext{
${ }^{7}$ It may also happen that the thermodynamically stable branch is only metastable, in which case the system jumps directly to the black hole branch without tachyon hair, and chiral symmetry is thus restored at this 1st order transition. The more complicated branch structure discussed in the next bullet may similarly contain metastable branches.
} 


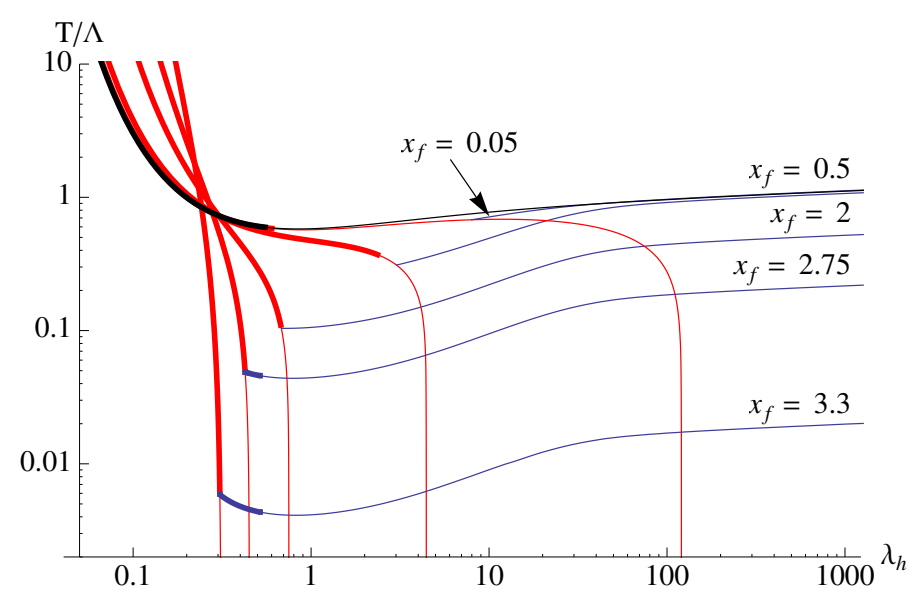

Figure 2. $T\left(\lambda_{h}\right) / \Lambda$ plotted for potentials of type II with SB normalisation (definitions specified in text) for various $x_{f}$ marked on figure. Thick curves represent stable phases. For more details at small $x_{f}$, see figure 22 .

give different physics, namely $0, \frac{12}{11}$, and $\frac{24}{11} .{ }^{\circ}$ We also consider $x_{f}$-dependent value, specified in (2.23) that corresponds to the normalization of the UV degrees of freedom of the free energy to the Stefan-Boltzmann limit of QCD.

- A final variation can be obtained on all of the above by using a glue potential $V_{g}(\lambda)$ $x_{f} V_{0 f}(\lambda)$ in (1.4) that has

(a) an extremum for all $x_{f}$ in the appropriate range, $\left.x_{f} \in\right] 0, \frac{11}{2}[$.

(b) an extremum only in part of this range, $x_{*}<x_{f}<\frac{11}{2}$. We will denote the potentials in this case by a star subscript.

According to the above options $\operatorname{PotI}_{*}\left(W_{0}=0\right)$ denotes a potential in the type I class, with $W_{0}=0$ and an IR critical point that exists only down to a finite $x_{*}$.

Let us then summarize the phase structure of the model as $x_{f}$ and the temperature are varied (at zero quark mass). In general one expects the phase diagram of figure 8 , so that for $0<x_{f}<x_{c}$ there is the 1st order transition at finite temperature, which also separates the chirally symmetric and broken phases. For $x_{f}>x_{c}$ the low temperature and high temperature configurations correspond to a tachyonless black holes, and, one expects a continuous crossover between these two.

For the various potentials presented above, this phase diagram is indeed obtained in the zeroth approximation, but for $x_{f}<x_{c}$ there are additional details which depend on the choice of potentials as follows.

- For potentials I the phase structure depends strongly on the choice for $W_{0}$ (see figure 18). For the lowest value $W_{0}=0$, there is only one 1st order transition

\footnotetext{
${ }^{8}$ Notice that the exactly zero value of $W_{0}$ is actually excluded, because it predicts wrong anomalous dimensions for quark mass or the chiral condensate [26]. We anyhow consider it as the limiting case of the allowed solutions. Moreover, $W_{0}$ may exceed the upper limit of $24 / 11$, if $x_{f}$ dependence is allowed.
} 
at $^{9} T=T_{d}$ for all $0<x_{f}<x_{c}$, except possibly for $x_{f}$ very close to $x_{c}$, where solving the phase diagram numerically becomes demanding. As $W_{0}$ is increased, a complicated structure appears near $x_{f}=x_{c}$, where we have two 1st order transitions between chirally broken phases, and the restoration of chiral symmetry at a 2 nd order transition at even higher temperature. At even higher $W_{0}$ the 1 st order transitions combine again into a single one, but the separate 2 nd order transition continues to exist for $x_{f}$ close to $x_{c}$. At low $x_{f}$, there is also a surprising change as $W_{0}$ increases. The chiral symmetry breaking phases disappear, but there is a 1st order transition between two chirally symmetric black hole phases at a finite temperature instead.

- For potentials II the dependence on $W_{0}$ is milder (see figures $13-16$ ). At high $W_{0}$, for low $x_{f}$ up to some value $x_{\chi}$, there is only the 1 st order transition at ${ }^{10} T=T_{d}$. When $x_{\chi}<x_{f}<x_{c}$, the chiral symmetry restoration takes again place at a 2 nd order transition at $T_{\chi}$ such that $T_{\chi}>T_{d}$. For decreasing $W_{0}, x_{\chi}$ increases, and finally disappears by joining with $x_{c}$.

- For the potentials $\mathrm{I}_{*}$, the phase structure is the standard one for high $x_{f}$, i.e., a 2nd order transition and a 1st order one with critical temperatures $T_{\chi}>T_{d}$ within a range $x_{\chi}<x_{f}<x_{c}$, with the former separating the chirally symmetric and broken phases (see figure 19). For lower $x_{f}$ there is only one 1st order transition. For $x_{f} \lesssim 2$, in the region where the effective potential does not admit an extremum, chiral symmetry is intact at all temperatures. We find a single 1st order transition between chirally symmetric thermal gas and black hole phases.

- For potentials $\mathrm{II}_{*}$, the phase structure is simple (see figure 17): there is a single 1st order transition for all $\left.x_{f} \in\right] 0, x_{c}$ [. In particular, the system is in a chirally broken phase at low temperatures, even in the region of low $x_{f}$ where the effective potential does not have an extremum.

\section{Defining V-QCD}

\subsection{Gravity action of the model}

The action of V-QCD is [26]

$$
S=M^{3} N_{c}^{2} \int d^{5} x \mathcal{L} \equiv \frac{1}{16 \pi G_{5}} \int d^{5} x \mathcal{L},
$$

where $^{11}$

$$
\begin{aligned}
\mathcal{L} & =\left[\sqrt{-g}\left(R-\frac{4}{3} \frac{(\partial \lambda)^{2}}{\lambda^{2}}+V_{g}(\lambda)\right)-V_{f}(\lambda, \tau) \sqrt{\operatorname{det}\left(g_{a b}+\kappa(\lambda, \tau) \partial_{a} \tau \partial_{b} \tau\right)}\right] \\
& =\sqrt{-g}\left[R+\left[-\frac{4}{3} g^{\mu \nu} \partial_{\mu} \phi \partial_{\nu} \phi+V_{g}(\lambda)\right]-V_{f}(\lambda, \tau) \sqrt{1+g^{r r} \kappa(\lambda(r)) \tau^{\prime}(r)^{2}}\right] .
\end{aligned}
$$

\footnotetext{
${ }^{9} T_{d}=T_{h}$ in figure 18.

${ }^{10} T_{\chi}=T_{\text {end }}$ of figures $13-16$ when it is in the stable brach.

${ }^{11}$ Notice that for notational simplicity we have absorbed a factor of $x_{f}$, which is visible in eq. (1.3), into $V_{f}(\lambda, \tau)$. See also eq. (2.10) below.
} 
The metric Ansatz is

$$
d s^{2}=b^{2}(r)\left[-f(r) d t^{2}+d \mathbf{x}^{2}+\frac{d r^{2}}{f(r)}\right], \quad b(r)=e^{A(r)} \underset{r \rightarrow 0}{\sim} \frac{\mathcal{L}_{\mathrm{UV}}}{r},
$$

and the two scalar functions, $1 / \lambda$ sourcing $F^{2}$ and $\tau$ sourcing $\langle\bar{q} q\rangle$, are

$$
\lambda=\lambda(r)=e^{\phi(r)}, \quad \tau=\tau(r) .
$$

In the second form $\sqrt{-g}$ has been factored out of the DBI action. The Gibbons-Hawking counter term would be

$$
S_{G H}=-\int d^{4} x \sqrt{-\gamma}\left[2 K+\frac{6}{\mathcal{L}_{\mathrm{UV}}}+\frac{\mathcal{L}_{\mathrm{UV}}}{2} R(\gamma)\right]
$$

with, for a hypersurface $r=$ const,

$$
K=\frac{\sqrt{f}}{2 b}\left(8 \frac{b^{\prime}(r)}{b}+\frac{f^{\prime}(r)}{f}\right) .
$$

Notice also that we have set the gauge fields $A_{L, R}$, which are dual to the left and right handed fermion currents, to zero, and neglected the Wess-Zumino terms. These terms do not affect the thermodynamics of the models.

The background solution of the dilaton $\lambda(r)$ and the warp factor $A(r)$ are identified as the 't Hooft coupling and the logarithm of the energy scale of the dual field theory, respectively [30-32]. As a matter of convention, we shall fix the normalisation of $\lambda(r)$ so that its relation to the perturbative QCD coupling is

$$
\lambda(r)=\frac{g^{2}(b(r))}{8 \pi^{2}} .
$$

The results of the model are independent of this normalisation, changing $\lambda \rightarrow \lambda / \lambda_{0}$ one simply has to change the potentials by $V(\lambda) \rightarrow V\left(\lambda_{0} \lambda\right)$. The convention of [26], for example, is obtained by shifting by $\lambda_{0}=1 /\left(8 \pi^{2}\right)$.

Important ingredients of the model are the relation of the bulk fields at $r$ to the QCD beta and quark mass anomalous dimension functions evaluated for a coupling at scale $b(r)$. Motivated by the connection to field theory, one defines

$$
\beta=\frac{d \lambda}{d b / b}=\lambda^{\prime}(A)=-b_{0} \lambda^{2}-b_{1} \lambda^{3}-b_{2} \lambda^{4} \ldots, \quad \gamma=\tau^{\prime}(A) .
$$

Matching with the perturbative expansion of the QCD beta function gives

$$
b_{0}=\frac{1}{3}\left(11-2 x_{f}\right), \quad b_{1}=\frac{1}{6}\left(34-13 x_{f}\right) .
$$

The action contains the gluonic potential $V_{g}(\lambda)$ and the fermionic potential $V_{f}(\lambda, \tau)$, which will be specified to the form

$$
V_{f}(\lambda, \tau)=x_{f} V_{f 0}(\lambda) e^{-a(\lambda) \tau^{2}}
$$

The detailed form of these and the functions $\kappa(\lambda), a(\lambda)$ will be discussed in the following subsections. 


\subsection{Construction of the potentials}

The potentials can be constructed in stages. First one fixes the potentials $V_{g}(\lambda)$ and $V_{f 0}(\lambda)$ up to order $\lambda^{2}$ in the UV, using the two scheme independent coefficients of the beta function. This analysis is simplified by the fact that the tachyon decouples in the UV. Next one fixes the UV behavior of the functions $a(\lambda)$ and $\kappa(\lambda)$, which parametrize the tachyon dependence of the action using the similarly scheme independent UV running properties of the quark mass and the condensate. Finally, one fixes the large $\lambda$ behavior of the potentials by requiring that the model reproduces known features of QCD in the IR, such as confinement, linear Regge trajectories, and reasonable zero-temperature phase structure. We shall discuss the various steps in detail below (see also [26]).

\subsubsection{The potentials from the beta function in the UV}

In the UV, since the tachyon vanishes much faster than the dilaton, we can first set it to zero. Then the dilaton profile can be linked to the effective potential $V_{\text {eff }}(\lambda)=V_{g}(\lambda)-$ $x_{f} V_{f 0}(\lambda)$ [26] by using Einstein's equations [30-32]. Defining $\beta=d \lambda / d \ln b=-b_{0} \lambda^{2}-b_{1} \lambda^{3}$, to order $\lambda^{2}$,

$$
\begin{aligned}
V_{g}-x_{f} V_{0 f} & =\frac{12}{\mathcal{L}_{\mathrm{UV}}^{2}} \exp \left[-\frac{8}{9} \int_{0}^{\lambda} d \lambda \frac{\beta}{\lambda^{2}}\right]\left(1-\frac{\beta^{2}}{9 \lambda^{2}}\right) \\
& =\frac{12}{\mathcal{L}_{\mathrm{UV}}^{2}}\left[1+\frac{8}{9} b_{0} \lambda+\left(\frac{23}{81} b_{0}^{2}+\frac{4}{9} b_{1}\right) \lambda^{2}\right] \\
& =V_{0}-x_{f} W_{0}+\left(V_{1}-x_{f} W_{1}\right) \lambda+\left(V_{2}-x_{f} W_{2}\right) \lambda^{2}
\end{aligned}
$$

where we expanded

$$
V_{g}=V_{0}+V_{1} \lambda+V_{2} \lambda^{2}+\mathcal{O}\left(\lambda^{3}\right), \quad V_{f 0}=W_{0}+W_{1} \lambda+W_{2} \lambda^{2}+\mathcal{O}\left(\lambda^{3}\right),
$$

and where we have introduced an $x_{f}$ dependent AdS radius

$$
\mathcal{L}_{\mathrm{UV}}=\mathcal{L}\left(x_{f}\right)
$$

Applying equation (2.12) for $x_{f}=0$ we have for the gluonic potential

$$
\begin{aligned}
V_{g} & =\frac{12}{\mathcal{L}_{0}^{2}}\left(1+\frac{8}{9} b_{0}^{\mathrm{YM}} \lambda+\frac{23\left(b_{0}^{\mathrm{YM}}\right)^{2}+36 b_{1}^{\mathrm{YM}}}{81} \lambda^{2}\right) \\
& =\frac{12}{\mathcal{L}_{0}^{2}}\left(1+\frac{88}{27} \lambda+\frac{4619}{729} \lambda^{2}\right),
\end{aligned}
$$

where $b_{i}^{\text {YM }}$ are the values of $b_{i}$ for $x_{f}=0$ and $\mathcal{L}_{0}=\mathcal{L}\left(x_{f}=0\right)$. In practice, one usually sets the (dimensionful) quantity $\mathcal{L}_{0}=1$. 
By using equations (2.12) and (2.13) one can now solve for the coefficients of the fermionic potential:

$$
\begin{aligned}
x_{f} \mathcal{L}_{0}^{2} W_{0} & =12\left(1-\frac{\mathcal{L}_{0}^{2}}{\mathcal{L}_{\mathrm{UV}}^{2}}\right) \\
x_{f} \mathcal{L}_{0}^{2} W_{1} & =\frac{32}{3}\left(b_{0}^{\mathrm{YM}}-b_{0} \frac{\mathcal{L}_{0}^{2}}{\mathcal{L}_{\mathrm{UV}}^{2}}\right)=\frac{12 \cdot 8}{27}\left[11-\left(11-2 x_{f}\right) \frac{\mathcal{L}_{0}^{2}}{\mathcal{L}_{\mathrm{UV}}^{2}}\right] \\
x_{f} \mathcal{L}_{0}^{2} W_{2} & =\frac{12}{81}\left[23\left(b_{0}^{\mathrm{YM}}\right)^{2}+36 b_{1}^{\mathrm{YM}}-\left(23 b_{0}^{2}+36 b_{1}\right) \frac{\mathcal{L}_{0}^{2}}{\mathcal{L}_{\mathrm{UV}}^{2}}\right] \\
& =\frac{12}{729}\left[4619-\left(4619-1714 x_{f}+92 x_{f}^{2}\right) \frac{\mathcal{L}_{0}^{2}}{\mathcal{L}_{\mathrm{UV}}^{2}}\right]
\end{aligned}
$$

These equations still involve one free parameter, which can be taken to be either $W_{0}$ or $\mathcal{L}_{\text {UV }}$. We shall study two ways to fix this parameter. First, we can take $W_{0}$ to be constant. In this case [26]

$$
0 \leq \mathcal{L}_{0}^{2} W_{0} \leq \frac{24}{11}
$$

and the $x_{f}$-dependent AdS radius is given by

$$
\mathcal{L}_{\mathrm{UV}}=\frac{\mathcal{L}_{0}}{\sqrt{1-\frac{1}{12} \mathcal{L}_{0}^{2} W_{0} \cdot x_{f}}} .
$$

Second, we can make a special $x_{f}$-dependent choice, which (as we shall show later) automatically normalises the free energy at large $T$ to Stefan-Boltzmann:

$$
\mathcal{L}_{\mathrm{UV}}=\mathcal{L}_{0}\left(1+\frac{7}{4} x_{f}\right)^{1 / 3}
$$

Further, we have to fix the $\lambda$ dependence of the functions $a(\lambda)$ and $\kappa(\lambda)$ in the tachyon part

$$
x_{f} V_{f 0}(\lambda) e^{-a(\lambda) \tau^{2}} \sqrt{1+g^{r r} \kappa(\lambda(r)) \dot{\tau}^{2}},
$$

of the action, where $g^{r r}=f / b^{2}$. The leading logarithmic term to the UV expansion of the tachyon should be (remember that the energy dimension of $\tau$ is -1 )

$$
\tau(r) / \mathcal{L}_{\mathrm{UV}}=m r(-\ln \Lambda r)^{-\frac{\gamma_{0}}{b_{0}}}=m r(-\ln \Lambda r)^{-\frac{3}{2 b_{0}}}
$$

to satisfy the scheme independent UV running of the quark mass. Here $\gamma_{0}=3 / 2$ is the leading coefficient of the anomalous dimension of the quark mass in QCD, $\gamma(\lambda)=\gamma_{0} \lambda+\cdots$. By using the tachyon equation of motion one sees that this requires that for small $\lambda$,

$$
\frac{\kappa(\lambda)}{a(\lambda)}=\frac{2}{3} \mathcal{L}_{\mathrm{UV}}^{2}\left[1-\left(\frac{8}{9} b_{0}+1\right) \lambda+\lambda^{2}+\cdots\right]
$$




\subsubsection{Large $\lambda$ behavior of the potentials}

To specify the full potential $V_{g}(\lambda)-x_{f} V_{f 0}(\lambda) e^{-a(\lambda) \tau^{2}}$ we have to continue the small $\lambda$ expansions to large $\lambda$. The guideline is quark confinement and chiral symmetry breaking at small $x_{f}$ and the appearance of an infrared fixed point at some $x_{f}=x_{c}$ (see [26]). Since there is no unique path to the result, we present the final forms of the potentials we use and motivate them.

We use the gluonic potential

$$
V_{g}(\lambda)=\frac{12}{\mathcal{L}_{0}^{2}}\left[1+\frac{88 \lambda}{27}+\frac{4619 \lambda^{2}}{729} \frac{\sqrt{1+\ln (1+\lambda)}}{(1+\lambda)^{2 / 3}}\right]
$$

which is constructed from the expansion (2.16) by simply multiplying the $\lambda^{2}$ term by the confinement factor

$$
\frac{\sqrt{1+\ln (1+\lambda)}}{(1+\lambda)^{2 / 3}}
$$

Then $V_{g}$ has the proper large- $\lambda$ behavior [30-32] but the small- $\lambda$ behavior is left intact. One could add scale factors of type $\lambda / \lambda_{0}$ containing more parameters.

For the fermionic potential $V_{f 0}$ in

$$
V_{f}(\lambda, \tau)=x_{f} V_{f 0}(\lambda) e^{-a(\lambda) \tau^{2}}
$$

we consider two different choices. The first one is obtained directly using (2.18)-(2.20)

$$
\begin{aligned}
V_{f 0}=\frac{12}{\mathcal{L}_{\mathrm{UV}}^{2} x_{f}}\left[\frac{\mathcal{L}_{\mathrm{UV}}^{2}}{\mathcal{L}_{0}^{2}}\right. & -1+\frac{8}{27}\left(11 \frac{\mathcal{L}_{\mathrm{UV}}^{2}}{\mathcal{L}_{0}^{2}}-11+2 x_{f}\right) \lambda \\
& \left.+\frac{1}{729}\left(4619 \frac{\mathcal{L}_{\mathrm{UV}}^{2}}{\mathcal{L}_{0}^{2}}-4619+1714 x_{f}-92 x_{f}^{2}\right) \lambda^{2}\right]
\end{aligned}
$$

Here one could as well use the parameter $W_{0}$ which is related to $\mathcal{L}_{\mathrm{UV}}$ by

$$
\frac{\mathcal{L}_{0}^{2}}{\mathcal{L}_{\mathrm{UV}}^{2}}=1-\frac{x_{f} \mathcal{L}_{0}^{2} W_{0}}{12} .
$$

For this choice the effective potential

$$
V_{\mathrm{eff}}(\lambda)=V_{g}(\lambda)-x_{f} V_{f 0}(\lambda)
$$

has a single maximum at finite positive $\lambda=\lambda_{*}$ for all $0<x_{f}<11 / 2$, indicating a (possible) infra-red fixed point.

The second choice is obtained introducing the confinement factor (2.28) also for the fermionic potential, i.e.,

$$
\begin{aligned}
V_{f 0}=\frac{12}{\mathcal{L}_{\mathrm{UV}}^{2} x_{f}}\left[\frac{\mathcal{L}_{\mathrm{UV}}^{2}}{\mathcal{L}_{0}^{2}}\right. & -1+\frac{8}{27}\left(11 \frac{\mathcal{L}_{\mathrm{UV}}^{2}}{\mathcal{L}_{0}^{2}}-11+2 x_{f}\right) \lambda \\
& \left.+\frac{1}{729}\left(4619 \frac{\mathcal{L}_{\mathrm{UV}}^{2}}{\mathcal{L}_{0}^{2}}-4619+1714 x_{f}-92 x_{f}^{2}\right) \lambda^{2} \frac{\sqrt{1+\ln (1+\lambda)}}{(1+\lambda)^{2 / 3}}\right]
\end{aligned}
$$


Now the effective potential has a maximum only at large $x_{f}$. To see this concretely, consider again the case (2.23). The asymptotic large- $\lambda$ behavior of $V_{g}-x_{f} V_{f 0}$ now is $\lambda^{4 / 3} \sqrt{\ln \lambda}$ times the function

$$
\frac{18476}{243}-4 \frac{4619\left(1+\frac{7}{4} x_{f}\right)^{2 / 3}-4619+1714 x_{f}-92 x_{f}^{2}}{243\left(1+\frac{7}{4} x_{f}\right)^{2 / 3}} .
$$

This function is positive for small $x_{f}$, negative at large $x_{f}(<11 / 2)$ and has a zero at $x_{f}=3.26817$. Thus there is a (possible) fixed point $\lambda_{*}$ only for $3.26817<x_{f}<11 / 2$.

Let us then discuss the IR behavior of the potentials $a$ and $\kappa$ which appear in the tachyon DBI action. For the function $\kappa$ we will consider the large- $\lambda$ asymptotics

$$
\kappa(\lambda) \underset{\lambda \rightarrow \infty}{\sim} \lambda^{-4 / 3} .
$$

This is motivated by the fact that in the action the combination $\kappa(\lambda) / b^{2}$ has the same asymptotics as $1 / b_{s}^{2}$ at large $\lambda$, where $b_{s}=b \lambda^{2 / 3}$ is the metric factor $b$ in the string frame. To ensure that the fractional exponent limit at large $\lambda$ does not spoil analyticity at small $\lambda$, we replace $\lambda^{4 / 3}$ by $(1+\# \lambda)^{4 / 3}$ in the expression for $\kappa(\lambda)$.

More precisely, two qualitatively different, acceptable choices for the IR asymptotics of $a$ (and $\kappa$ ) were identified in [26]. These are produced by the following two choices. The first choice has

$$
a(\lambda)=\frac{3}{2} \frac{1}{\mathcal{L}_{\mathrm{UV}}^{2}}, \quad \kappa(\lambda)=\frac{1}{\left[1+\frac{3}{4}\left(\frac{8}{9} b_{0}+1\right) \lambda\right]^{4 / 3}}=\frac{1}{\left(1+\frac{115-16 x_{f}}{36} \lambda\right)^{4 / 3}},
$$

and leads to tachyon growing exponentially at large $r$,

$$
\tau(r) \underset{r \rightarrow \infty}{\sim} \tau_{0} e^{C r}
$$

where $C$ is a known constant (see appendix B) and $\tau_{0}$ parametrises the solutions. The second choice is given by

$$
\kappa(\lambda)=\frac{1}{(1+\lambda)^{4 / 3}}, \quad a(\lambda)=\kappa(\lambda) \frac{3}{2 \mathcal{L}_{\mathrm{UV}}^{2}}\left[1+\left(\frac{8}{9} b_{0}+1\right) \lambda+\lambda^{2}\right]
$$

and for them the leading divergence is

$$
\tau(r) \underset{r \rightarrow \infty}{\sim} C \sqrt{r-r_{1}}
$$

where the constant $C$ is again known and now $r_{1}$ parametrises the solutions. To select this solution, it is required that the last term in the square brackets in (2.38) grows faster than $\lambda^{4 / 3}$.

Finally, let us summarize our choices for acceptable potentials. We always keep $V_{g}$ fixed to the expression (2.27) and choose $V_{f 0}, a$, and $\kappa$ as follows:

- Potentials $I$ : We take $V_{f 0}$ as in equation (2.30), so that the fixed point $\lambda_{*}$ exists for all $0<x_{f}<11 / 2$. For $a$ and $\kappa$ we use the choice of equations (2.36), which lead to exponentially diverging tachyon in the IR. 
- Potentials $I I$ : We take again $V_{f 0}$ from equation (2.30), but use the other choice (2.38) for $a$ and $\kappa$. Then the tachyon diverges as $\tau \sim \sqrt{r}$ in the IR.

- Potentials $I_{*}$ : We use now the fermionic potential $V_{f 0}$ of equation (2.33), which contains the confinement factor. Thus the extremum exists only within the interval $3.26817<x_{f}<11 / 2$. For $a$ and $\kappa$ we use the choice of equations (2.36), which lead to exponentially diverging tachyon in the IR.

- Potentials $I I_{*}$ : We use $V_{f 0}$ with the confinement factor, but use the other choice (2.38) for $a$ and $\kappa$. Then the fixed point exist only for large $x_{f}$, and the tachyon diverges as $\tau \sim \sqrt{r}$ in the IR.

To fully pin down the potentials, we also need to specify the value of $W_{0}$ (or $\mathcal{L}_{\mathrm{UV}}$ ) which is used. We choose four reference values:

- $W_{0}=0$ (and constant). This is the lower bound of $W_{0}$. Actually, exactly zero $W_{0}$ is not acceptable because the anomalous dimensions of the quark mass and the chiral condensate do not sum up to zero. This case is nevertheless interesting as it is the limit of acceptable solutions.

- $W_{0}=12 / 11$. This is the standard choice studied in [26].

- $W_{0}=24 / 11$. For constant $W_{0}$, this is the largest possible value, for which $\mathcal{L}_{\mathrm{UV}} \rightarrow \infty$ as $x_{f} \rightarrow 11 / 2$.

- $W_{0}\left(\right.$ and $\left.\mathcal{L}_{\mathrm{UV}}\right)$ fixed such that the free energy automatically matches with the standard Stefan-Boltzmann result at high temperature with the correct number of degrees of freedom (see eq. (2.23) and the discussion in section 3.4 below).

An ongoing work [70] studies the meson spectra in this model. As it turns out, the potentials $\mathrm{I}$ and $\mathrm{I}_{*}$ admit linear "Regge" trajectories, so that the quadratic masses are asymptotically linear in the excitation number, $m_{n}^{2} \sim n$, independently of the other quantum numbers. Potentials II and $\mathrm{II}_{*}$, however, have linear trajectories only in the glueball sector, while the other trajectories are quadratic, $m_{n}^{2} \sim n^{2}$. As linear trajectories are expected in QCD, this observation favors potentials $\mathrm{I}$ and $\mathrm{I}_{*}$.

\subsubsection{IR fixed point and the BF bound for the tachyon}

Now that the potentials are defined, one can check that they satisfy an important requirement: they permit the determination of the bulk tachyon mass and, equating this with the Breitenlohner-Freedman (BF) instability bound, the determination of the start of the conformal window. Take $\tau(r)=0$ (there is no chiral symmetry breaking in the conformal window) and note that at small $\lambda, V_{g}(\lambda)-x_{f} V_{f 0}(\lambda)>0$. However, $V_{f 0}(\lambda)$ grows faster and the conformal window starts at the value $\lambda_{*}$ defined by the vanishing derivative

$$
V_{g}^{\prime}\left(\lambda_{*}\right)-x_{f} V_{f 0}^{\prime}\left(\lambda_{*}\right)=0
$$




\begin{tabular}{|c|c|c|c|c|}
\hline & PotI & PotI $_{*}$ & PotII & PotII $_{*}$ \\
\hline$W_{0}=0$ & 4.10209 & 4.33334 & 4.17825 & 4.38493 \\
$W_{0}=12 / 11$ & 3.99591 & 4.33334 & 4.07968 & 4.38493 \\
$W_{0}=24 / 11$ & 3.71607 & 4.33334 & 3.80086 & 4.38493 \\
$W_{0}$ SB & 3.59172 & 4.33334 & 3.70008 & 4.38493 \\
\hline
\end{tabular}

Table 1. The critical values $x_{c}$ for the various potentials. Notice that for the types $\mathrm{I}_{*}$ and $\mathrm{II}_{*}, x_{c}$ is independent of $W_{0}$.

Given $\lambda_{*}$ one defines an IR AdS radius

$$
\frac{12}{\mathcal{L}_{\mathrm{IR}}^{2}}=V_{g}\left(\lambda_{*}\right)-x_{f} V_{f 0}\left(\lambda_{*}\right), \quad \mathcal{L}_{\mathrm{UV}}>\mathcal{L}_{\mathrm{IR}}
$$

The tachyon mass at $\lambda_{*}$ in units of $\mathcal{L}_{\mathrm{IR}}$ becomes

$$
-m_{\mathrm{IR}}^{2} \mathcal{L}_{\mathrm{IR}}^{2}=\frac{24 a\left(\lambda_{*}\right)}{\kappa\left(\lambda_{*}\right)\left[V_{g}\left(\lambda_{*}\right)-x_{f} V_{f 0}\left(\lambda_{*}\right)\right]}
$$

Gravity solutions with $\tau=0$ are stable when $m_{\mathrm{IR}}^{2} \mathcal{L}_{\mathrm{IR}}^{2}>-4$; the conformal window thus starts when (2.42), as a function of $x_{f}$, has the value 4 .

Eq. (2.42) can be evaluated for the two choices of $a, \kappa$ above. For the choice (2.36) (types $\mathrm{I}$ and $\mathrm{I}_{*}$ ) the equation becomes

$$
\frac{36\left[1+\frac{1}{36}\left(115-16 x_{f}\right) \lambda_{*}\right]^{4 / 3}}{\mathcal{L}_{\mathrm{UV}}^{2}\left[V_{g}\left(\lambda_{*}\right)-x_{f} V_{f 0}\left(\lambda_{*}\right)\right]}=4 .
$$

For the choice $(2.38)$ (types II and $\left.\mathrm{II}_{*}\right)$, the $x_{c^{-}}$equation $(2.42)$ has the form

$$
\frac{36\left[1+\frac{1}{27}\left(115-16 x_{f}\right) \lambda_{*}+\lambda^{* 2}\right]}{\mathcal{L}_{\mathrm{UV}}^{2}\left[V_{g}\left(\lambda_{*}\right)-x_{f} V_{f 0}\left(\lambda_{*}\right)\right]}=4 .
$$

The values of $x_{c}$ can then be calculated by inserting the potential $V_{g}-x_{f} V_{f 0}$ and the chosen value for $W_{0}$ in these equations. The critical values for the potentials listed above are given in table 1.

The $x_{f}$-dependence of the tachyon mass for all the potential choices suggested above is shown in figure 3 . The critical value $x_{c}$ is the rightmost point where the curve intersects the horizontal dashed line where the BF bound is saturated. For potentials $\mathrm{I}_{*}$ and $\mathrm{II}_{*}$ (solid magenta curves) the fixed point only exists for $x_{*}<x_{f}<11 / 2$ with $x_{*} \simeq 3.27$. In this case the tachyon mass diverges as $x$ approaches $x_{*}$ from above.

From (2.43) and (2.44) one sees, using the asymptotics of the potentials (see eq. (4.3) below), that $-m_{\mathrm{IR}}^{2} \mathcal{L}_{\mathrm{IR}}^{2} \sim 1 / \sqrt{\ln \left(1 / x_{f}\right)}$ for type I and $-m_{\mathrm{IR}}^{2} \mathcal{L}_{\mathrm{IR}}^{2} \sim 1 / x_{f}$ for type II as $x_{f} \rightarrow 0$. They thus behave completely differently in this limit, for type I the mass vanishes, for type II it grows without bounds. In particular, for potentials I and for low $x_{f}$ the (absolute value of the squared) tachyon mass dives below the BF bound. This means that the existence of a solution with a nontrivial tachyon profile and zero quark mass is 

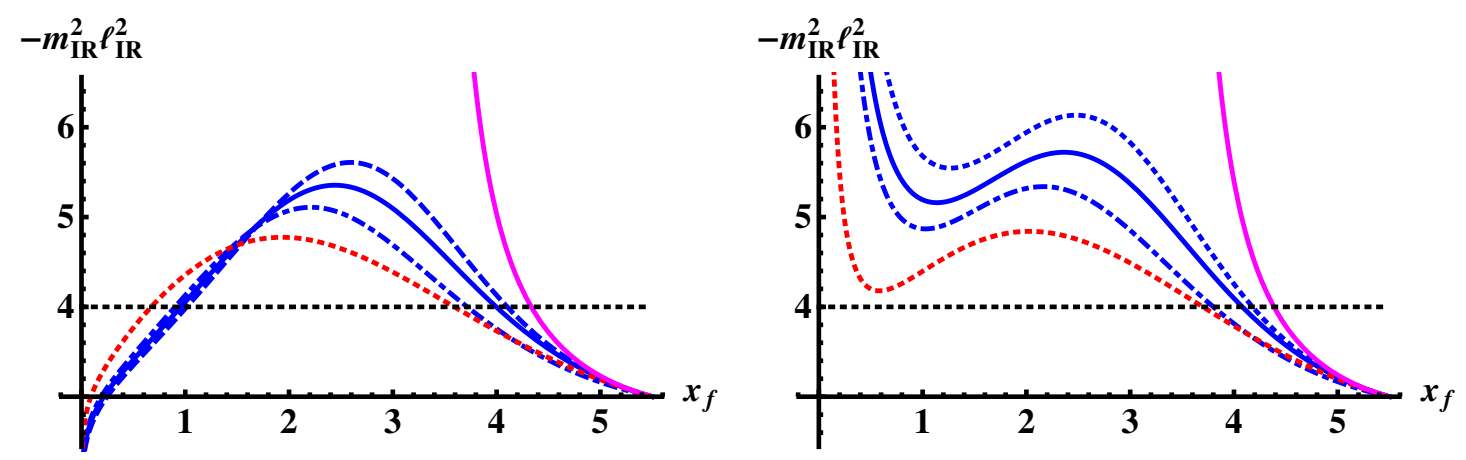

Figure 3. The squared tachyon mass at the IR fixed point, see eqs.(2.43) and (2.44). Left: potentials I; Right: potentials II. The blue curves give the masses for constant $W_{0}$. The dashed, solid and dotdashed curves have $W_{0}=0,12 / 11$, and 24/11, respectively. The dotted red curves have $W_{0}$ fixed according to the Stefan-Boltzmann normalization of the free energy in the UV. The solid magenta (uppermost) curves are for potentials $\mathrm{I}_{*}$ and $\mathrm{II}_{*}$, for which the tachyon mass is independent of $W_{0}$. The black dotted horizontal line marks the BF bound.

not guaranteed [26], which means that chiral symmetry could remain intact even at low temperatures. However, in most of the cases, such a solution anyhow exists all the way down to $x_{f}=0$, and the expected picture with chiral symmetry breaking is obtained. We shall discuss this issue in more detail below.

\section{V-QCD at finite temperature: equations and their solution}

The V-QCD action has two kinds of vacua at finite temperature, either with identically vanishing tachyon or with nontrivial tachyon profile. The tachyonless black hole solutions can be constructed in the same way as in the Yang-Mills case [33-35]. Below most of the discussion will in principle assume the presence of the tachyon, but the construction for the solutions without the tachyon can be obtained simply by setting $\tau=0$ everywhere.

\subsection{Equations and numerical solution}

The goal now is to find numerical solutions of the Einstein's equations for the metric functions $b(r)=e^{A(r)}, f(r)$ and the scalars $\lambda(r), \tau(r)$, satisfying

$$
f\left(r_{h}\right)=0, \quad f(0)=1, \quad b(r) \underset{r \rightarrow 0}{\sim} \frac{\mathcal{L}_{\mathrm{UV}}}{r},
$$

where $r_{h}$ marks the location of the horizon.

Due to the singular behavior of the solutions near the UV boundary $(r \rightarrow 0)$, it proves to be convenient to use $A=\ln b$ as a coordinate instead of $r$ in the numerical solution. Carrying out this transformation, one finds that the combination

$$
q(A)=e^{A} \frac{d r}{d A}=-\frac{1}{W}
$$

appears naturally. This is just a rewriting of the superpotential

$$
W=-\frac{\dot{b}}{b^{2}}=-e^{-A} \frac{d A}{d r}
$$


The equations of motion then become

$$
\begin{aligned}
& 12-6 \frac{q^{\prime}}{q}+\frac{4}{3} \frac{\lambda^{\prime 2}}{\lambda^{2}}+3 \frac{f^{\prime}}{f}=\frac{q^{2}}{f}\left(V_{g}-V_{f} \sqrt{1+f \kappa \tau^{\prime 2} / q^{2}}\right), \\
& 12-\frac{4}{3} \frac{\lambda^{\prime 2}}{\lambda^{2}}+3 \frac{f^{\prime}}{f}=\frac{q^{2}}{f}\left(V_{g}-\frac{V_{f}}{\sqrt{1+f \kappa \tau^{\prime 2} / q^{2}}}\right) \\
& 4-\frac{q^{\prime}}{q}+\frac{f^{\prime \prime}}{f^{\prime}}=0 \\
& \tau^{\prime \prime}+\left(4-\frac{q^{\prime}}{q}+\frac{f^{\prime}}{f}+\lambda^{\prime} \frac{\partial \ln \kappa}{\partial \lambda}+\lambda^{\prime} \frac{\partial \ln V_{f}}{\partial \lambda}\right) \tau^{\prime}=-\frac{f \kappa}{q^{2}}\left(4+\frac{f^{\prime}}{2 f}+\frac{\lambda^{\prime}}{2} \frac{\partial \ln \kappa}{\partial \lambda}+\lambda^{\prime} \frac{\partial \ln V_{f}}{\partial \lambda}\right) \tau^{\prime 3} \\
& +\frac{\partial \ln V_{f}}{\partial \tau} \tau^{\prime 2}+\frac{q^{2}}{f \kappa} \frac{\partial \ln V_{f}}{\partial \tau}, \\
& \frac{\lambda^{\prime \prime}}{\lambda}+\frac{f^{\prime}}{f} \frac{\lambda^{\prime}}{\lambda}+4 \frac{\lambda^{\prime}}{\lambda}-\frac{\lambda^{\prime 2}}{\lambda^{2}}-\frac{q^{\prime}}{q} \frac{\lambda^{\prime}}{\lambda}=-\frac{3}{8} \frac{q^{2} \lambda}{f}\left(\frac{\partial V_{g}}{\partial \lambda}-\frac{\partial V_{f}}{\partial \lambda} \sqrt{1+\frac{f}{q^{2}} \kappa \tau^{\prime 2}}-\frac{f}{2 q^{2}} \frac{V_{f} \frac{d \kappa}{d \lambda} \tau^{\prime 2}}{\sqrt{1+\frac{f}{q^{2}} \kappa \tau^{\prime 2}}}\right) .
\end{aligned}
$$

Here the prime denotes differentiation with respect to $A$. Near the UV boundary $r=0$,

$$
A=\ln b=\ln \frac{\mathcal{L}_{\mathrm{UV}}}{r} \rightarrow+\infty .
$$

The range of $A$ thus is $A_{h}<A<+\infty$, where $A_{h}$ is the horizon,

$$
f\left(A_{h}\right)=0 .
$$

Numerical integration starts by solving $q^{\prime}, \lambda^{\prime}, f^{\prime \prime}, \tau^{\prime \prime}$ from the four first ones in terms of lower derivatives; the fifth equation, the equation for $\lambda$, will be used as a check and constraint. For brevity we introduce two square root factors:

$$
R_{1}=\sqrt{1+\frac{f \kappa}{q^{2}} \tau^{\prime 2}}
$$

and

$$
R_{2}=\sqrt{12+\frac{3 f^{\prime}}{f}-\frac{q^{2}}{f}\left(V_{g}-\frac{V_{f}}{R_{1}}\right)} .
$$

The equations to be solved numerically then are

$$
\begin{aligned}
q^{\prime}= & q\left[4+\frac{f^{\prime}}{f}-\frac{q^{2}}{6 f}\left(2 V_{g}-V_{f} R_{1}-\frac{V_{f}}{R_{1}}\right)\right] \\
\lambda^{\prime}= & -\frac{\sqrt{3}}{2} \lambda R_{2} \\
f^{\prime \prime}= & f^{\prime}\left[\frac{f^{\prime}}{f}-\frac{q^{2}}{6 f}\left(2 V_{g}-V_{f} R_{1}-\frac{V_{f}}{R_{1}}\right)\right]=f^{\prime}\left(\frac{q^{\prime}}{q}-4\right) \\
\tau^{\prime \prime}= & -\frac{q^{2}}{6 f}\left(2 V_{g}-V_{f} R_{1}-\frac{V_{f}}{R_{1}}\right) \tau^{\prime}-\frac{f \kappa}{q^{2}}\left(4+\frac{f^{\prime}}{2 f}\right) \tau^{\prime 3} \\
& +\frac{\sqrt{3}}{2}\left(\tau^{\prime}+\frac{f \kappa}{2 q^{2}} \tau^{\prime 3}\right) \frac{\lambda \partial_{\lambda} \kappa}{\kappa} R_{2}+\frac{\sqrt{3}}{2}\left(\tau^{\prime}+\frac{f \kappa}{q^{2}} \tau^{\prime 3}\right) \frac{\lambda \partial_{\lambda} V_{f}}{V_{f}} R_{2}+\left(\frac{q^{2}}{f \kappa}+\tau^{\prime 2}\right) \frac{\partial_{\tau} V_{f}}{V_{f}}
\end{aligned}
$$


In the $\lambda$ equation the minus branch has to be chosen as $\lambda(A)$ is a monotonically decreasing function of $A$. The derivatives are with respect to $A$. The equations are autonomous in the sense that there is no explicit $A$ dependence. Numerical integration then proceeds as follows:

1. Let us fix the horizon at $A=A_{h}=-\epsilon$, where $\epsilon$ is a sufficiently small number, e.g., $\epsilon=10^{-6}$. the values of the functions at $A=0$, which is taken as the initial value of numerical integration, are computed by using the expansions (B.19)(B.22) in appendix B. These numbers can now be obtained by inserting the values of $\lambda_{h}, \tau_{h}, f_{h}^{\prime}$. Among these the horizon values of the scalars, $\lambda_{h}, \tau_{h}$, remain as parameters, $f_{h}^{\prime}$ can be given an arbitrary positive value, +1 , say. One then finds a solution $q_{1}(A), f_{1}(A), \lambda_{1}(A), \tau_{1}(A)$ valid from $A=0$ to some large upper limit $A_{+}$ by using NDSolve of Mathematica. The spatial coordinate $r(A)$ can then, if needed, be computed by similarly integrating the differential equation

$$
r^{\prime}(A)=e^{-A} q(A)
$$

with the initial condition $r(A=\infty)=0$.

2. The so obtained first-level solution $f_{1}(A)$ is scaled to one in the $\mathrm{UV}(A \rightarrow \infty)$ by writing $f_{2}(A)=f_{1}(A) / f_{1}\left(A_{+}\right)$. Simultaneously $q_{2}(A)=q_{1}(A) / \sqrt{f_{1}\left(A_{+}\right)}$, which is needed since eq. (3.4) demands that $q^{2} / f$ be invariant. Finally, $\lambda_{2}=\lambda_{1}, \tau_{2}=\tau_{1}$.

3. The final scaling is performed to guarantee that all solutions use the same unit of energy or, equivalently, have the same integration constant in the integral of the definition (2.8) of the beta function. This implies

$$
A-\hat{A}_{0}=\ln (b)-\hat{A}_{0}=\frac{1}{b_{0} \lambda(A)}+\frac{b_{1}}{b_{2}^{2}} \ln \left(b_{0} \lambda(A)\right)+\left(\frac{b_{2}}{b_{0}^{2}}-\frac{b_{1}^{2}}{b_{0}^{3}}\right) \lambda(A)+\mathcal{O}\left(\lambda^{2}\right),
$$

where $\hat{A}_{0}$ is the integration constant. By inserting the UV expansions of $A$ and $\lambda$ from appendix A, we identify $\hat{A}_{0}=\ln \left(\mathcal{L}_{\mathrm{UV}} \Lambda\right)$. We wish to scale $\Lambda$ to one ${ }^{12}$, and therefore define

$$
A_{0}=\hat{A}_{0}-\ln \mathcal{L}_{\mathrm{UV}}=\ln \Lambda,
$$

and shift solutions by $A_{0}$. In practice, one implements this by determining, for a given numerical solution (the $\mathcal{O}\left(\lambda_{2}\right)$ term is optional),

$$
A_{0}=\lim _{A \rightarrow \infty}\left[A-\ln \mathcal{L}_{\mathrm{UV}}-\frac{1}{b_{0} \lambda_{2}(A)}-\frac{b_{1}}{b_{0}^{2}} \ln \left(b_{0} \lambda_{2}(A)\right)-\left(\frac{b_{2}}{b_{0}^{2}}-\frac{b_{1}^{2}}{b_{0}^{3}}\right) \lambda_{2}(A)\right]
$$

and then performing the scaling

$$
\lambda_{3}(A)=\lambda_{2}\left(A+A_{0}\right)
$$

\footnotetext{
${ }^{12}$ After this, all quantities are expressed in units of $\Lambda$; omitting the factor $\mathcal{L}_{\mathrm{UV}}$ would give a unit of energy depending on $x_{f}$
} 
etc. for all the functions at level 2. The set $q_{3}(A), f_{3}(A), \lambda_{3}(A), \tau_{3}(A)$, parametrised by the values of $\lambda_{h}, \tau_{h}$, is the final numerical solution. Note that the horizon has now been shifted to

$$
A_{h}=-A_{0}-\epsilon \approx-A_{0}
$$

at level 2 it was defined by $f_{2}(-\epsilon)=0$.

\subsection{Physical quantities}

The set of functions $q(A), f(A), \lambda(A), \tau(A)$ (leaving out the index 3 ) can now be converted to various physical quantities:

The temperature is

$$
T=-\frac{1}{4 \pi} f^{\prime}\left(r_{h}\right)=-\left.\frac{e^{A}}{4 \pi q(A)} f^{\prime}\left(A_{h}\right)\right|_{A_{0}=A_{h}}=\frac{e^{-A_{0}}}{-4 \pi q\left(-A_{0}\right)} f^{\prime}\left(-A_{0}\right),
$$

and the value of $b$ at the horizon is

$$
b_{h}=e^{-A_{0}} .
$$

The quark mass $m_{q}$ is defined by the UV expansion of the tachyon:

$$
\tau(r)=\mathcal{L}_{\mathrm{UV}} m_{q}(-\ln \Lambda r)^{-\frac{9}{22-4 x_{f}}} r
$$

so that, using the relation (3.18),

$$
m_{q}=\lim _{A \rightarrow \infty} \mathcal{L}_{\mathrm{UV}}^{-1} \tau(A) \exp \left[\frac{1}{b_{0} \lambda(A)}+\left(\frac{b_{1}}{b_{0}^{2}}-\frac{9}{22-4 x_{f}}\right) \ln \left(b_{0} \lambda(A)\right)\right]
$$

In practice, the extrapolation to $A=\infty$ can be carried out by measuring $\tilde{m}_{q}(A)$, as defined by the right hand side of eq. (3.26), at two large values of $A$ and then linearly extrapolating to $\lambda=0$ :

$$
m_{q}=\frac{\tilde{m}_{q}\left(A_{1}\right) \lambda\left(A_{2}\right)-\tilde{m}_{q}\left(A_{2}\right) \lambda\left(A_{1}\right)}{\lambda\left(A_{2}\right)-\lambda\left(A_{1}\right)} .
$$

Linear extrapolation is chosen, because the leading neglected terms in the expansion of eq. (3.26) are (up to logarithmic corrections) linear in $\lambda$.

\subsection{Fixing quark mass}

The above is for fixed $\lambda_{h}, \tau_{h}$. The really demanding task is to find the field configurations at fixed $m_{q}$. For this one needs the curves $\tau_{h}\left(\lambda_{h}, m_{q}\right)$. The quark mass is determined by the UV behavior of the tachyon: $\tau(r) / \mathcal{L}_{\mathrm{UV}} \simeq m_{q}(-\ln r)^{-\gamma_{0} / b_{0}} r$. To fix $m_{q}$ at fixed $\lambda_{h}$ we have to solve the equations of motion at various $\tau_{h}$ and find that value of $\tau_{h}$ which leads to the desired UV behavior of $\tau(r)$. 

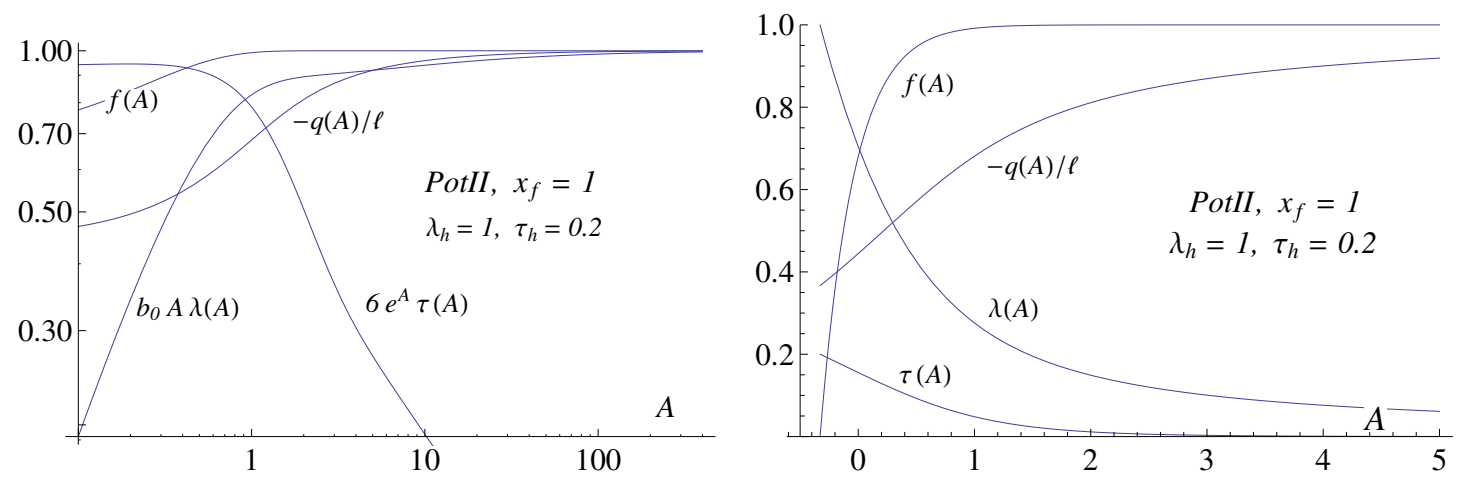

Figure 4. Explicit bulk configurations. Left: UV large- $A$ region. Right: near horizon region. For this configuration $T=0.3839, b_{h}=0.7200, m_{q}=0.05422$.

\subsubsection{Zero quark mass}

In particular, we are interested in $m_{q}=0$. This case splits in two parts: either $\tau(r)=0$ identically (chiral symmetry holds) or $\tau(r)$ nonzero (chiral symmetry broken).

If $\tau=0$, solutions with $m_{q}=0$ are obtained simply by setting $\tau_{h}=0$ above. The solution is then controlled by the effective potential $V_{g}(\lambda)-x_{f} V_{f 0}(\lambda)$. For classes I and II, this increases monotonically from $\lambda=0$, but since $V_{f 0}$ grows faster, the derivative decreases and becomes finally zero at some $\lambda=\lambda_{*}\left(x_{f}\right)$ (see eq. (2.40)). The extremum of the potential marks the location of the IR fixed point, which is screened by the horizon at finite temperature. Indeed, the tachyonless black holes have $0<\lambda_{h}<\lambda_{*}$, and for $\lambda_{h}$ very close to $\lambda_{*}$ we obtain configurations where the dilaton is approximately constant, $\lambda \simeq \lambda_{h} \simeq \lambda_{*}$ for a long range of the coordinate before the horizon is reached in the deep IR.

For classes $\mathrm{I}_{*}$ and $\mathrm{II}_{*}$, the effective potential $V_{g}(\lambda)-x_{f} V_{f 0}(\lambda)$ does not have an extremum for $x_{f}$ below $x_{*} \simeq 3.27$. In this case the fixed point is absent, and the tachyonless black hole solutions are qualitatively similar to Yang-Mills $\left(x_{f} \rightarrow 0\right)$ [30-32]. In particular $\lambda_{h}$ can take any value.

For non-zero $\tau(r)$, the discussion of $m_{q}=0$ configurations has to take into account the existence of Efimov zeroes, oscillatory behavior when approaching $r=0$, which was discussed above in the introduction. We discuss here the standard picture which is seen in most cases for $x_{f}<x_{c}$. A rough description of more complicated cases is given in appendix C. The situation is summarised in figure 5. For large $\tau_{h}>0, \tau(r)$ decreases monotonically from $\tau_{h}$ towards $r=0$ and ends with positive $m_{q}$. We evaluate $m_{q}$ using (3.27) with two large values of $A$ (corresponding to a small UV cutoff $\epsilon$ in the $r$-coordinate). When $\tau_{h}$ is decreased, ultimately an (approximate) $m_{q}=0$ configuration $\left(\tau_{0}\left(r, m_{q}=0\right)\right.$ in figure 5$)$ with monotonically decreasing $\tau(r)$ is obtained.

This defines the curve $\tau_{h 0}\left(\lambda_{h}\right)$ in figure 6 . One finds that these solutions are possible only if $\lambda_{h}$ is larger than a fixed positive value, which we call $\lambda_{\text {end }}$. Decreasing $\tau_{h}$ further, $\tau(r)$ first develops a zero so that $m_{q}<0$. Continuing even further we find a second location where $m_{q}=0$ vanishes. This is a configuration with one tachyon node $\left(\tau_{1}\left(r, m_{q}=0\right)\right.$ in figure 5$)$. The pattern continues with an ever increasing number of nodes, until one ends up 


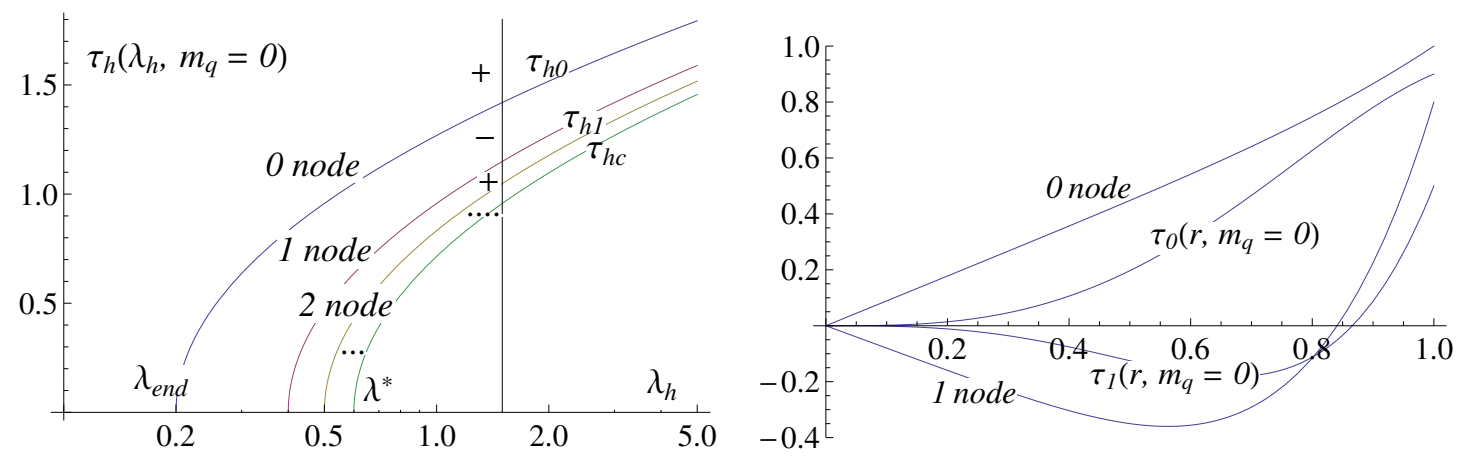

Figure 5. Left: schematic presentation of the curves $\tau_{h}\left(\lambda_{h}, m_{q}=0\right)$, i.e., those values of the horizon value $\tau_{h}$ of the tachyon which lead to configurations with $m_{q}=0$, at that particular $\lambda_{h}$. Choosing $\tau_{h}$ on the curve $\tau_{h i}$ leads to a $\tau(r)$ without a linear term and with $i$ zeros at some $r$. The zero mass solutions with vanishing tachyon live on the line $0<\lambda_{h}<\lambda_{*}$. The plus and minus signs indicate the sign of the quark mass in each region limited by the curves $\tau_{h 0}, \tau_{h 1}, \ldots$ Right: schematic presentation of the $r$ dependence of the bulk tachyon for low node numbers. Tachyon solutions for top to bottom are: a generic solution with $m_{q}>0$ and no nodes ("0 node"), the standard solution with zero quark mass $\left(\tau_{0}\right)$, the solution with zero quark mass and one node $\left(\tau_{1}\right)$, and a generic solution with $m_{q}<0$ and one node ("1 node"). See the text for a more detailed explanation.

with the curve $\tau_{h c}\left(\lambda_{h}\right)$, below which a solution with the standard UV boundary does not exist. Numerically, the curves $\tau_{h 0}\left(\lambda_{h}\right)$ and $\tau_{h 1}\left(\lambda_{h}\right)$ can be separated, but already $\tau_{h 2}\left(\lambda_{h}\right)$ would require so much effort that we have not embarked on computing it. As we approach the conformal window, the curves $\tau_{h 0}, \tau_{h 1}, \ldots$ get closer and closer to $\tau_{h c}$ and finally vanish for $x_{f} \geq x_{c}$.

We expect that increasing the number of nodes increases the free energy so that to study equilibrium states it is enough to compute $\tau_{h 0}\left(\lambda_{h}, m_{q}=0\right)$. This was checked at zero temperature in [26] numerically for potentials I, and analytically in the limit $x_{f} \rightarrow x_{c}$ as well as in the limit of large number of tachyon nodes.

\subsubsection{Nonzero quark mass}

For nonzero quark mass the special solution with identically vanishing tachyon profile is missing. However, there are solutions of various types for $\tau_{h}>0$, as suggested by figure 5 . We shall here restrict to the "standard" solutions which have monotonic tachyon, i.e., the region above $\tau_{h 0}$ in figure 5 (left). Below this curve there can be Efimov type solutions where the tachyon has nodes. As for $m_{q}=0$, we expect that these solutions have higher free energies than the standard one. In the region of standard solutions, the dependence of quark mass is smooth (see figure 6). We have found numerically that for fixed $\lambda_{h}$ the correspondence between $m_{q}$ and $\tau_{h}$ is one-to-one. Therefore $m_{q}$ can be kept fixed by following a set of well-defined curves on the $\left(\lambda_{h}, \tau_{h}\right)$-plane, some of which are sketched in figure 6 .

It is also interesting to notice how the $m_{q}=0$ solution is obtained from the ones having finite quark masses as $m_{q} \rightarrow 0$. What happens for nonzero quark mass is shown in figure 6 for a concrete computation. If $0<\lambda_{h}<\lambda_{\text {end }}$ (and $\lambda_{h}$ fixed), the curve $\tau_{h}\left(\lambda_{h}, m_{q}\right)$ 


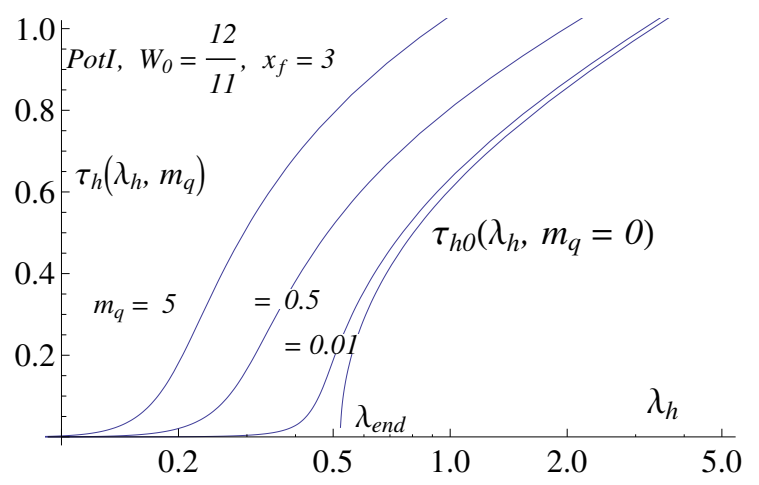

Figure 6. The curves $\tau_{h}\left(\lambda_{h}, m_{q}\right)$ for values of $m_{q}$ marked in the figure, computed for Potential I with $W_{0}=12 / 11$. Here $\lambda_{\text {end }}=0.5221$ and $\lambda_{*}=0.6467$.

approaches zero as $m_{q} \rightarrow 0$, indicating that $\tau(r)$ approaches the chiral symmetry conserving solution $(\tau(r) \equiv 0)$ uniformly. If $\lambda_{h}>\lambda_{\text {end }}, \tau_{h}\left(\lambda_{h}, m_{q}\right)$ approaches $\tau_{h 0}\left(\lambda_{h}\right)$ instead, which implies that $\tau(r)$ converges to the standard chiral symmetry breaking solution $\tau_{0}(r)$.

\subsection{Thermodynamics}

We now want to compute minus free energy density or pressure $p\left(T, m_{q} ; x_{f}\right)$ of the gravity dual, assuming that all the quarks have the same mass $m_{q}$. In particular, we are interested in $m_{q}=0$. The chemical potential is zero, there is an equal number of quarks and antiquarks. The equilibrium phase has the largest pressure.

The basic strategy is to compute the temperature and entropy density from the formulas

$$
T=-\frac{1}{4 \pi} f^{\prime}\left(r_{h}\right), \quad s=\frac{1}{4 G_{5}} b^{3}\left(r_{h}\right),
$$

where $f$ and $b$ are obtained by solving Einstein's equations. The pressure is then obtained by integrating $s(T)=p^{\prime}(T)$. The key technical issues are keeping track of the quark mass and specifying the integration constant in the pressure integral.

The general structure of temperature (for a case containing a fixed point) is shown in figure 7 , to be consulted in association with figures 5 and 6 . For $m_{q}=0$ two branches separate. Firstly, for $0<\lambda_{h}<\lambda_{*}$ there is the temperature computed for chirally symmetric vanishing tachyon solutions. We shall use the notation $T_{u}\left(\lambda_{h}\right) \equiv T\left(\lambda_{h}, \tau_{h}=0\right)$ for this temperature below.

The chiral symmetry breaking solution exists for $\lambda_{\text {end }}<\lambda_{h}<\infty$ and as $\lambda_{h} \rightarrow \lambda_{\text {end }}$, the corresponding temperature curve ends precisely on the curve which has identically vanishing tachyon. The temperature curve is computed by using the zero node zero mass curve $\tau_{h 0}\left(\lambda_{h}\right)$ in figure 5 . We shall use the notation $T_{b}\left(\lambda_{h}\right) \equiv T\left(\lambda_{h}, \tau_{h 0}\left(\lambda_{h}, m_{q}=0\right)\right.$ ) for this temperature. If we computed the temperature for the one node solution $\tau_{h 1}\left(\lambda_{h}\right)$, we would get a curve which lies significantly below the zero node curve in figure 7 and again ends on the zero tachyon curve. These solutions will have a higher free energy and we can thus neglect them. 

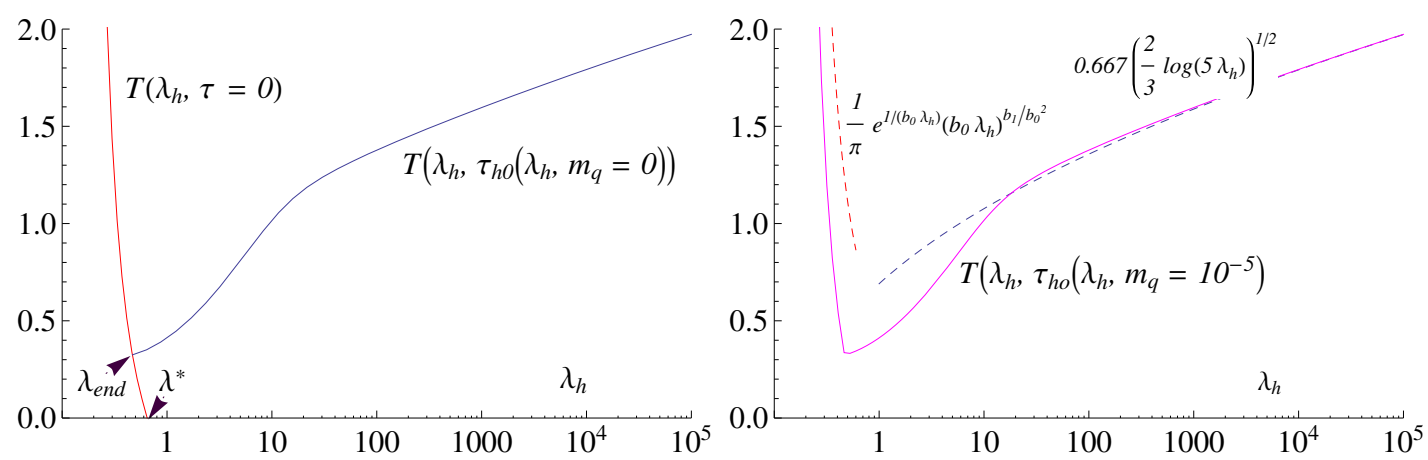

Figure 7. The temperature as a function of $\lambda_{h}$ for solutions for Pot II at $x_{f}=3$ and $W_{0}=12 / 11$, both for zero (Left) and very small mass (Right). The asymptotic limits (3.29) and (3.30) are also shown for $m_{q}=10^{-5}$, in the range of the figure the UV limit is not yet accurate. The maximum value $\lambda^{*}$ of $\lambda$ for the $\tau=0$ curve is defined in (2.40). See also figure 2 .

Whenever the quark mass is nonzero, the tachyon cannot be vanishing and that branch disappears. However, as seen from figures 6 and 7 , the small-mass curve very closely approximates the zero tachyon curve, also at small $\lambda_{h}$.

Analytic approximations are often useful. In the UV $f(r) \rightarrow 1-r^{4} / r_{h}^{4}$ so that

$$
\pi T\left(\lambda_{h}\right)=\frac{1}{r_{h}}=e^{1 /\left(b_{0} \lambda_{h}\right)}\left(b_{0} \lambda_{h}\right)^{b_{1} / b_{0}^{2}}=\frac{b\left(\lambda_{h}\right)}{\mathcal{L}_{\mathrm{UV}}} .
$$

Similarly, in the IR (see (B.10) in appendix B),

$$
T\left(\lambda_{h}\right) \sim\left(\frac{2}{3} \ln \lambda_{h}\right)^{1 / 2}, \quad b\left(\lambda_{h}\right) \sim \frac{1}{\lambda_{h}^{2 / 3}}\left(\frac{2}{3} \ln \lambda_{h}\right)^{1 / 4}
$$

For a numerical check, see figure 7. The interesting physics takes place in the region connecting these two limits.

The function $b\left(\lambda_{h}\right)$ decreases monotonically while the function $T\left(\lambda_{h}\right)$ decreases in the UV but starts increasing in the IR. The physics of the UV increase is obvious, this is the weak coupling limit which naturally corresponds to large $T$ of a thermal fluid. The (extremely slow) increase in the IR is a quantitative fact but does not correspond to a stable phase. This is simplest seen by computing the sound velocity

$$
c_{s}^{2}=\frac{d p}{d \epsilon}=\frac{s}{T s^{\prime}(T)}=\frac{b\left(\lambda_{h}\right)}{3 T\left(-b^{\prime}\left(\lambda_{h}\right)\right)}\left(-\frac{d T}{d \lambda_{h}}\right)
$$

A stable phase has $c_{s}^{2}>0$ (equivalently, has a positive specific heat) and this requires $T^{\prime}\left(\lambda_{h}\right)<0$. Thus only the UV decreasing part can correspond to a stable phase, the IR part is the unstable small black hole region, small since $s \sim b^{3}\left(\lambda_{h}\right) \rightarrow 0$ there. It is, nevertheless, crucially important for the phase structure. 
To compute the pressure, we have to integrate the entropy density (3.28) over $T$. Taking $\lambda_{h}$ as a variable, we have integrals over the two branches in figure 7 :

$$
\begin{aligned}
& p_{b}(T)=\frac{1}{4 G_{5}} \int_{\lambda_{h}(T)}^{\infty} d \lambda_{h}\left(-T_{b}^{\prime}\left(\lambda_{h}\right)\right) b_{b}^{3}\left(\lambda_{h}\right)+p_{b}(\infty), \\
& p_{u}(T)=\frac{1}{4 G_{5}} \int_{\lambda_{h}(T)}^{\lambda_{*}} d \lambda_{h}\left(-T_{u}^{\prime}\left(\lambda_{h}\right)\right) b_{u}^{3}\left(\lambda_{h}\right)+p_{u}\left(\lambda_{*}\right),
\end{aligned}
$$

where $b, u$ refer to the chiral symmetry broken $\left(\tau_{h}=\tau_{h 0}\left(\lambda_{h}, m_{q}=0\right)\right)$ and chirally symmetric (or unbroken, $\tau=0$ ) phases. The continuity of pressure at $T_{\text {end }}=T\left(\lambda_{\text {end }}\right)$ leads to a rather remarkable consistency check of the entire scheme: it demands

$$
\frac{1}{4 G_{5}} \int_{\lambda_{\text {end }}}^{\lambda_{*}} d \lambda_{h}\left(-T_{u}^{\prime}\left(\lambda_{h}\right)\right) b_{u}^{3}\left(\lambda_{h}\right)-\frac{1}{4 G_{5}} \int_{\lambda_{\text {end }}}^{\infty} d \lambda_{h}\left(-T_{b}^{\prime}\left(\lambda_{h}\right)\right) b_{b}^{3}\left(\lambda_{h}\right)=p_{b}(\infty)-p_{u}\left(\lambda_{*}\right) .
$$

However, the difference on the r.h.s. is nothing but the difference between the free energies of the broken and symmetric phases at $T=0$ :

$$
p_{b}(\infty)-p_{u}\left(\lambda_{*}\right)=-F_{b}(T=0)+F_{u}(T=0) .
$$

This difference was computed in [26] from the $T=0$ solutions, with no black hole. Here they are computed in (3.34) from the black hole solutions and we have checked numerically that the results agree within the numerical precision.

The computation of the free energy now proceeds as follows, first for the simple structure of $T\left(\lambda_{h}\right)$ in figure 7 :

- Start by integrating (3.32) from some large value of $\lambda_{h}$ down to $\lambda_{\text {end }}$, choosing $p_{b}(\infty)=0$. Since $T^{\prime}\left(\lambda_{h}\right)>0$ in figure 7 , this leads to a negative pressure. This is not the stable phase, the physical stable phase is not described by this metric. The stable phase with the largest pressure is the thermal gas phase with $p=0$.

- At $\lambda_{\text {end }}$ move to the chirally symmetric $\tau=0$ branch and fix the constant $p_{u}\left(\lambda_{*}\right)$ by demanding continuity of pressure. Since now $T^{\prime}\left(\lambda_{h}\right)<0, p$ starts increasing. At first $p$ is still negative and the stable phase is the thermal gas phase with $p=0$.

- At some $\lambda_{h} \equiv \lambda_{c}$ pressure passes through 0. This defines a transition temperature $T_{h}$ since from now on the black hole metric has the largest pressure. Since $\tau=0$ this black hole phase is chirally symmetric.

- The latent heat of the transition is

$$
\frac{L}{T_{h}^{4}}=\frac{s\left(T_{h}\right)}{T_{h}^{3}}=\frac{1}{4 G_{5}}\left(\frac{b\left(\lambda_{h}\right)}{T_{h}}\right)^{3}<N_{c}^{2} \frac{4 \pi^{2}}{45}\left(1+\frac{7}{4} x_{f}\right),
$$

where the maximum value is obtained taking normalisation from (3.39) and using the UV approximation (A.19). Counting degrees of freedom one has $N_{f}^{2}$ Goldstone bosons in the low $T$ phase (for which we do not have a $T$ dependent gravity dual) and $2 N_{c}^{2}+\frac{7}{2} N_{c} N_{f}$ degrees of freedom in the high $T$ phase. These are equal at $x_{f}=4$ and if latent heat is naively assumed to be proportional to the jump in the number of degrees of freedom, one might rather expect $L$ to decrease when $x_{f}$ increases. 
- Asymptotically, for large $T, \lambda_{h} \rightarrow 0$ we have $\pi T=1 / r_{h}=b\left(\lambda_{h}\right) / \mathcal{L}_{\mathrm{UV}}$ so that

$$
4 G_{5} p=\left(\pi \mathcal{L}_{\mathrm{UV}}\right)^{3} \int_{\lambda_{h}}^{\infty} d x\left(-T^{\prime}(x)\right) T^{3}(x)=\frac{1}{4}\left(\pi \mathcal{L}_{\mathrm{UV}}\right)^{3} T^{4} .
$$

If one for large $T$ assumes that the system becomes a gas of non-interacting bosons and fermions one should have

$$
\frac{p}{T^{4}}=\left(1+\frac{7}{4} x_{f}\right) \frac{\pi^{2}}{45} N_{c}^{2} .
$$

This is obtained from (3.33) if

$$
\frac{1}{4 G_{5}}=\frac{4}{45 \pi} \frac{1+\frac{7}{4} x_{f}}{\mathcal{L}_{\mathrm{UV}}^{3}} N_{c}^{2},
$$

which can be used to normalise the pressure.

- The above was for the simple $T\left(\lambda_{h}\right)$ in figure 7 . Depending on the potentials, more complex structures can appear, as analysed in the following section.

- To present results for $p / T^{4}$ we choose to normalise it so that it approaches at large $T$ the ideal gas Stefan-Boltzmann pressure according to (3.38). However, we have no dynamical argument for fixing the $x_{f}$ dependence of $\mathcal{L}_{\mathrm{UV}}$ in (3.39). We shall present the phase diagrams for two choices, for the automatically SB-normalised case (see eq. (3.39))

$$
\mathcal{L}_{\mathrm{UV}}=\left(1+\frac{7}{4} x_{f}\right)^{1 / 3}, \quad W_{0}=\frac{12}{x_{f}}\left[1-\frac{1}{\left(1+\frac{7}{4} x_{f}\right)^{2 / 3}}\right]
$$

and for the $W_{0}$ fixed case

$$
\mathcal{L}_{\mathrm{UV}}=\frac{1}{\sqrt{1-\frac{1}{12} W_{0} x_{f}}}, \quad W_{0}=0, \frac{12}{11}, \frac{24}{11}
$$

In the former case one simply has

$$
\frac{1}{4 G_{5}}=\frac{4}{45 \pi} N_{c}^{2}
$$

and in the latter case $\mathrm{e}^{13}$

$$
\frac{1}{4 G_{5}}=\frac{4}{45 \pi} \frac{1+\frac{7}{4} x_{f}}{\left(1-\frac{1}{12} x_{f} W_{0}\right)^{2 / 3}} N_{c}^{2}
$$

the factor $N_{c}^{2}$ is furthermore often implied, i.e., results for $p /\left(N_{c}^{2} T^{4}\right)$ are given.

\footnotetext{
${ }^{13}$ Notice that in this case the glue part of the V-QCD action will also depend on $x_{f}$ through the normalization factor $1 / 4 G_{5}$.
} 


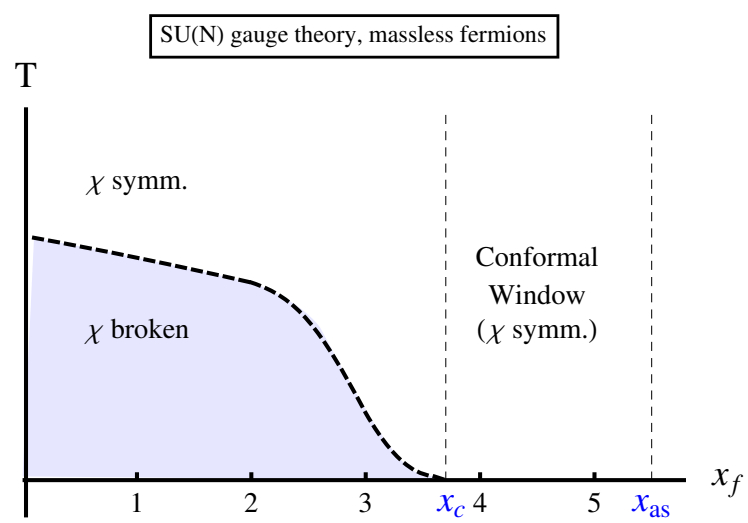

Figure 8. Qualitative behavior of the transition temperature between the low and high $T$ phases of $\mathrm{V}$-QCD matter.

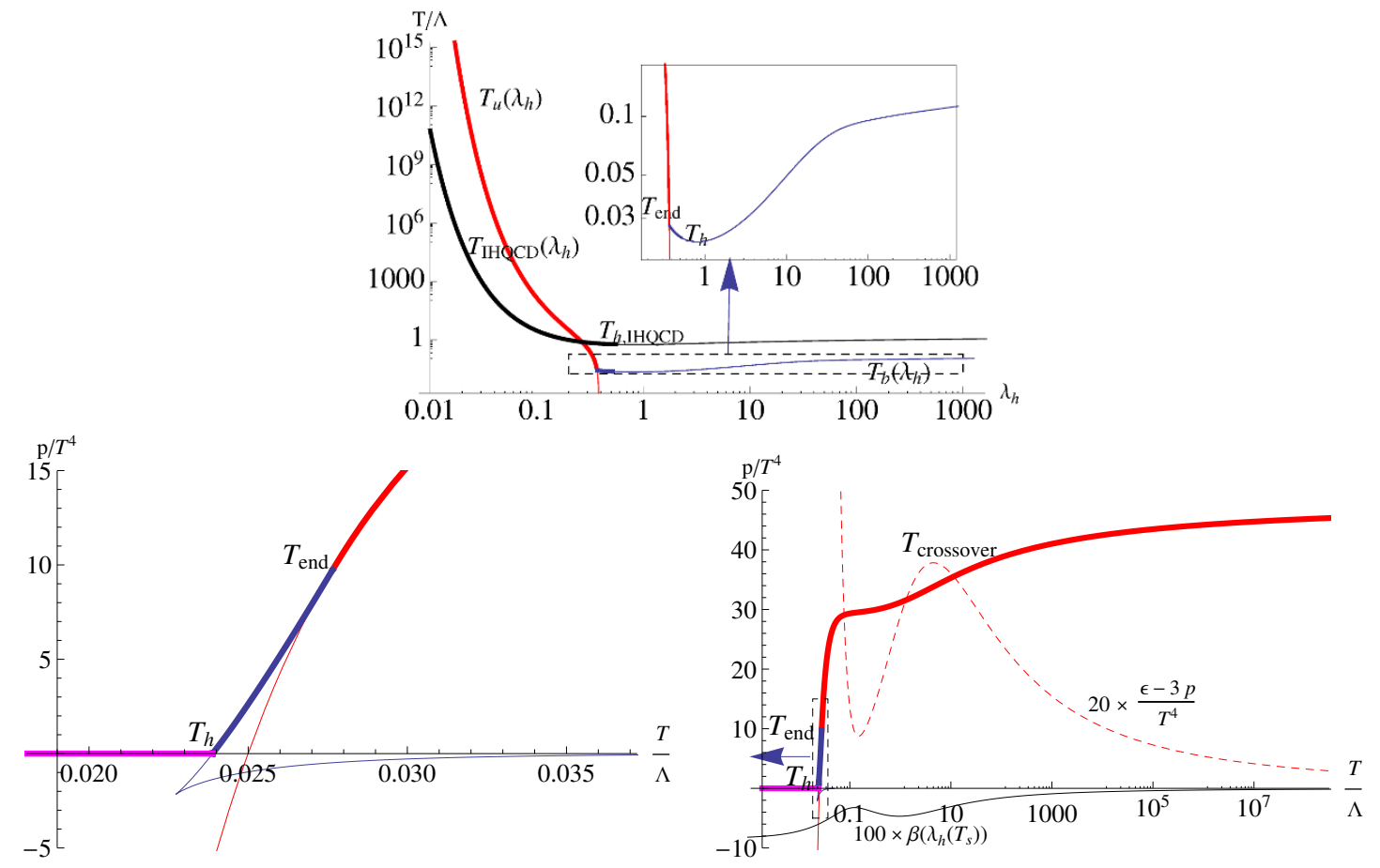

Figure 9. Examples of the $T_{\text {end }}, T_{h}$ and $T_{\text {crossover }}$ transitions in potential II with Stefan-Boltzmann -normalization of $\mathcal{L}_{\mathrm{UV}}$ and with $x_{f}=3$ (see also figure 13). Upper: The temperature $T\left(\lambda_{h}\right)$. The curving of $T_{u}\left(\lambda_{h}\right)$ at $\lambda_{h} \sim 0.2, T \sim 2$ is related to the crossover. The inset shows the minimum of $T_{b}\left(\lambda_{h}\right)$, which causes $p_{b}$ to be positive between $T_{h}$ and $T_{\text {end }}$. For comparison, we also plot $T\left(\lambda_{h}\right)$ for IHQCD with $x_{f}=0$. Lower left: $p / T^{4}$ in a close-up around the region of the $T_{h}$ and $T_{\text {end }}$-transitions. Lower right: an overview of the pressure in the same case, also showing the interaction measure, the peak of which determines the position of $T_{\text {crossover }}$. The black curve shows the vacuum beta function, scaled to fit, as a function of temperature in the symmetric phase, so that $\beta(T)=\beta\left(\lambda_{u}(T)\right)$, where $\lambda_{u}(T)$ is the inverse function of $T_{u}\left(\lambda_{h}\right)$. The walking maximum of the beta function clearly coincides with the plateau related to $T_{\text {crossover }}$, confirming that the $p / T^{4} \sim$ constant

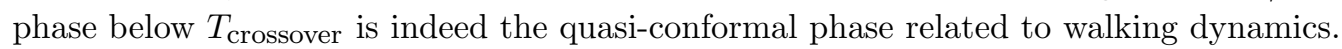




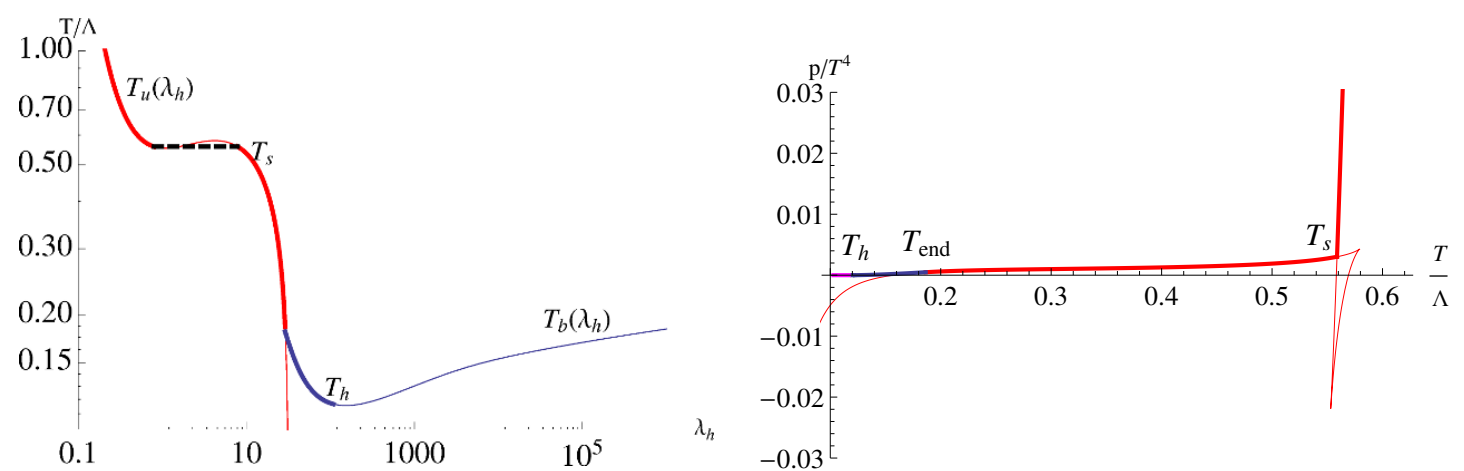

Figure 10. An example of the $T_{\mathrm{s}}$ transition in potential I with $W_{0}=24 / 11$ and with $x_{f}=0.3$ Left: the local maximum and minimum which generate the 1st order $T_{\mathrm{S}}$-transition. Right: $p(T) / T^{4}$ in the region around which the 1 st order $T_{\mathrm{S}}$ transition takes place, extending to smaller $T$ in order to show the relation to the $T_{h}$ and $T_{\text {end }}$ transitions.

\section{Results for the phase structure}

\subsection{Phase transitions}

Let us first review what one qualitatively expects for the phase structure of V-QCD when the number of (massless) fermions is changed [71]. This is shown in figure 8, where the transition temperature between a low $T$ and a high $T$ phase is plotted as a function of $x_{f}$.

A few reminders are in order. In the absence of quarks, YM has a $Z_{N_{c}}$ center symmetry that is central in the definition of the confined and deconfined phases. The relevant order parameter is the Polyakov loop that transforms nontrivially under $Z_{N_{c}}$. If its expectation value is zero, we are in the confined phase, while the expectation value becomes non-zero in the deconfined phase.

This expectation value is simple to calculate holographically, [72]. It corresponds to a string world-sheet along the time circle, and hanging down straight in the holographic (radial) direction. The important difference is where it ends. At zero temperature, this worldsheet extends to $r \rightarrow \infty$ and is the world-sheet of a free quark. Standard renormalization subtracts its contribution completely and therefore the Polyakov loop vev is zero (to leading order in $1 / N_{c}$ ) in the zero temperature phase.

In a regular black-hole phase, the worldsheet terminates at the horizon and after subtraction the Polyakov loop expectation value is non-zero. This is in agreement with the identification of black-hole phases generically as deconfined phases.

In the presence of massless quarks, the center symmetry is not a symmetry any more, and the Polyakov loop is not an order parameter. However at large $N_{c}$, there is alternative order parameter for a deconfined phase, namely the $N_{c}$ dependence of the free energy, $F$. In the confined phases $F \sim \mathcal{O}(1)$ while in deconfined phases, $F \sim \mathcal{O}\left(N_{c}^{2}\right)$. Again, with this criterion, the vacuum solutions (without horizons) are "confining" $(F \sim \mathcal{O}(1))$ while any black hole solution with regular horizon is "deconfined" $\left(F \sim \mathcal{O}\left(N_{c}^{2}\right)\right)$. It is therefore natural to use this criterion in our analysis in order to define deconfined phases.

The true symmetry in the case of massless quarks is chiral symmetry. This always has an order parameter, the chiral condensate, that distinguishes chirally symmetric from chirally broken phases. 
Given the remarks above, we summarize what we would expect.

- For $x_{f}=0$ one has the Yang-Mills 1st order phase transition between a confined and deconfined phase. In the high $T$ deconfined phase, the $Z_{N_{c}}$ symmetry is broken.

- For a somewhat higher $x_{f}$ one expects that there still is a 1st order transition. However, now this transition will involve chiral symmetry breaking/restoration.

- For $x_{f}$ approaching $x_{c}$ one expects the transition temperature to decrease rapidly as follows from Miransky scaling.

- For $x_{f}$ in the conformal window, $x_{c}<x_{f}<11 / 2$, both the low and high $T$ phases are conformal ones, which can be separated by a crossover. The only transition happens at $T=0^{+}$like in the AdS black hole in Poincaré coordinates.

The models we consider contain the full fermion backreaction and therefore predict a somewhat more detailed phase structure. New phase transitions of different orders can take place, lines can split in two, etc. The behavior in the conformal window $\left(x_{c}<x_{f}<11 / 2\right)$ is nonetheless always simple: there are no transitions, but a crossover between the low and high temperature conformal phases. Therefore we concentrate first on the phase structure in the region below the conformal transition $\left(x_{f}<x_{c}\right)$.

While the details of the phase structure depend on the choice of potential, the various phase transitions encountered appear in certain systematic ways. We will define a consistent notation, and describe the classes of transitions, assuming the system is heated up and we go from low temperatures to high temperatures.

To motivate the notation, we first list the various transitions and the corresponding temperatures.

- $T_{h}$ is the analogue of the QCD hadronisation transition if it is the chiral restoration transition (chirally symmetric $\rightarrow$ chirally broken).

- $T_{\text {end }}$ is the end point of the curve $T_{b}\left(\lambda_{h}\right)=T\left(\lambda_{h}, \tau_{h 0}\left(\lambda_{h}, m_{q}=0\right)\right)$, which contains the black holes with tachyon hair. For values of $\lambda_{h}$ smaller than at this endpoint, the black-holes have no tachyon hair.

- $T_{\text {crossover }}$ marks the position of a crossover. This crossover is defined by the position of the peak in the equation-of-state $\left((\epsilon-3 p) / T^{4}\right)$ as a function of temperature.

- $T_{\mathrm{s}}$ takes place at small $x_{f}$ within the chirally symmetric phase when one can jump from one decreasing branch of $T_{u}\left(\lambda_{h}\right)$ (no tachyon hair) to another.

- Finally $T_{12}$ involves the splitting of one 1 st order line to two.

With this notation we may now describe in detail the various types of transitions and crossovers we have found, and show examples of each case. In the figures we denote the stable phases with thick lines and meta- and unstable phases with thin lines. 
- The 1st order hadronisation transition at $T_{h}$, happens either between the chirally broken $\rightarrow$ a chirally symmetric phase (see figure 7 ) or from a chirally broken $\rightarrow$ a chirally broken phases (see figure 9). ${ }^{14}$ As described above, our normalization for pressure is such that the pressure of the $(T=0)$ hadron gas phase is zero. In the holographic setup, this transition is between that of the black hole phases, whose pressure remains positive down to the lowest temperature, and the hadron gas phase. The transition takes place at the temperature $T_{h}$ where the pressure of the $\mathrm{BH}$ phase reaches zero. Whether this phase is chirally symmetric or non-symmetric depends on the potential choices and $x_{f}$. For an example, see figure 13 .

- The 2 nd order chirally broken $\rightarrow$ chirally symmetric transition at $T_{\text {end }}=T\left(\lambda_{\text {end }}, 0\right)=$ $T\left(\lambda_{\text {end }}, \tau_{h 0}\left(\lambda_{\text {end }}, m_{q}=0\right)\right)$, see figures 7 or 9 . Since the chiral symmetry breaking solution starts to exist only above some $\lambda_{\text {end }}$, the system makes at that point a transition to the chirally symmetric phase. However, this transition may be absent in the thermodynamic limit: if $p_{b}\left(\lambda_{h}\right)$ is everywhere negative, the transition is between two thermodynamically metastable phases, and the relevant saddle point is never dominant. We denote the temperature of the transition by $T_{\text {end }}$. Since this transition takes place at one single value $\lambda_{h}=\lambda_{\text {end }}$, both pressure and entropy density are continuous $\left(b\left(\lambda_{h}\right)\right.$ does not jump). Therefore, only $p^{\prime \prime}(T)$ or $c_{s}^{2}$ are discontinuous, and the transition is of second order.

- The high- $T$ chirally symmetric $\rightarrow$ chirally symmetric crossover at $T_{\text {crossover }}$, see figure 9. This is a crossover which is expected on general grounds when $x_{f}$ is near but below $x_{c}$. It reflects the change of the dynamics from the walking region, where the QCD coupling constant evolves slowly, to the region in the deep UV where it runs. In this sense, above the crossover it is the nontrivial fixed point theory that controls the thermodynamics, while below the crossover it is the YM-like theory that controls the dynamics.

The thermodynamics behaves as follows: At first $p / T^{4}$ stabilizes to some intermediate value, before eventually increasing very slowly toward the Stefan-Boltzmann limit. For the potentials studied here, this creates a clear, although very broad, peak in the interaction measure, and the position of that peak can be used to define the temperature $T_{\text {crossover }}$ at which there is a crossover. The peak of the interaction measure is also observed at low values of $x_{f}$. In this region, however, $T_{\text {crossover }}$ is typically relatively close to $T_{h}$. Note also that for $\mathrm{SU}\left(N_{c}\right)$ YM theory, $N_{f}=0$ the interaction measure starts decreasing immediately at $T_{h}$ [73], $T_{\text {crossover }} \simeq T_{h}$.

- The 1 st order high-T chirally symmetric $\rightarrow$ chirally symmetric transition at $T_{\mathrm{s}}$, see figure 10: With some choices of potential, at low $x_{f}, T_{u}\left(\lambda_{h}\right)$ in the chirally symmetric (unbroken) part of the solution develops a local maximum and minimum. There are then two values of $\lambda_{h}$ between which both the pressure and the temperature of the

\footnotetext{
${ }^{14}$ There is also the special case of potentials $\mathrm{I}_{*}$ at low $x_{f}$ where the transition analogous to $T_{h}$ takes place from a chirally symmetric thermal gas to chirally symmetric black hole phase (see figure 19).
} 


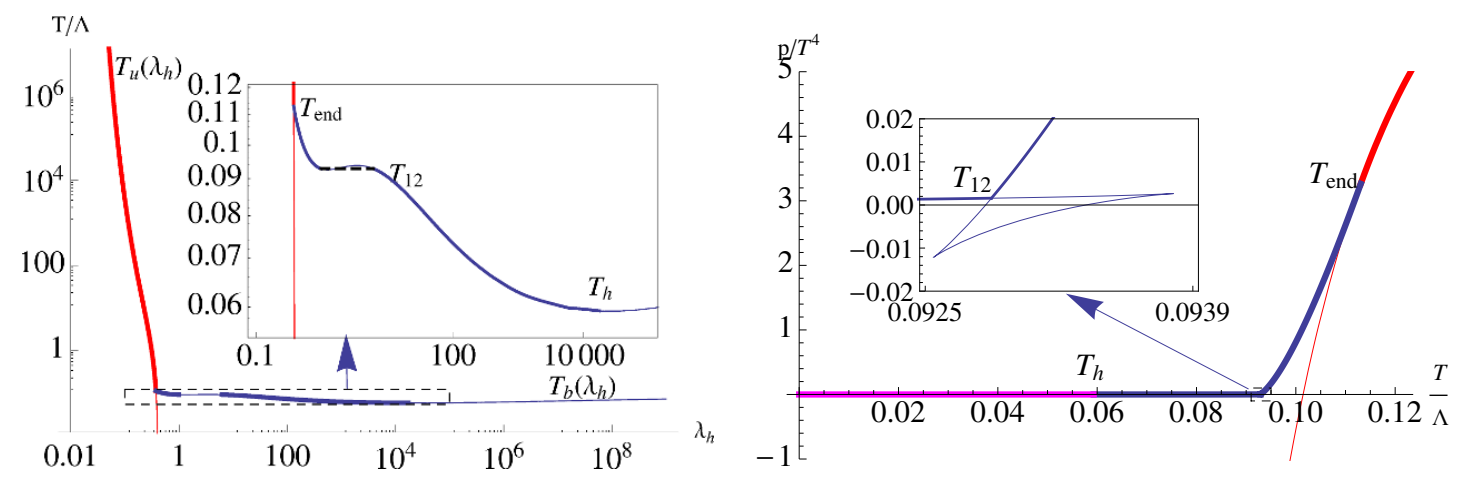

Figure 11. An example of the $T_{12}$ transition in potential I with $W_{0}=12 / 11$ and with $x_{f}=3.5$. Left: the overall structure of $T\left(\lambda_{h}\right)$, with an inset showing the maximum and minimum in more detail. Right: a close-up of $p(T) / T^{4}$ in the region where the $T_{12}$-transition happens, with an inset showing further detail.

solution match, and there is a 1st order transition between these two branches of the chirally symmetric solution. Interestingly, $T_{s}$ approaches the temperature of the YM transition in IHQCD as $x_{f} \rightarrow 0$ (see the discussion in section 4.8).

- The 1 st order chirally broken $\rightarrow$ chirally broken transition at $T_{12}$, see figure 11 . This happens in the chirally non-symmetric phase, with potential I and $W_{0}=12 / 11$, $T\left(\lambda_{h}\right)$ which develops a local minimum and maximum at large $x_{f}$. This again induces a 1 st order transition, which we denote by $T_{12}$. In this case the single 1 st order transition at $T_{h}$ splits into two 1st order transitions as $x_{f}$ increases above some critical value. Above this value, the transition with higher (lower) temperature is identified as $T_{12}\left(T_{h}\right)$.

\subsection{Class-II potentials}

Let us then discuss the details of the phase structure for the various potentials and choices of $W_{0}$ defined in section 2.2.2.

We take Class-II first since it leads systematically to a simple phase structure. We observe two possibilities: First, for $x_{f}$ up to some value $x_{\chi}<x_{c}$ the 1 st order deconfinement and chiral transition temperatures coincide, $T_{d}=T_{\chi}$, from this value up to $x_{c}$ one has $T_{\chi}>T_{d}$ and the higher chiral transition is of 2 nd order. Second, $T_{d}=T_{\chi}$ all the way up to $x_{c}$ and $x_{\chi}$ is absent.

For this choice of potentials the tachyon diverges $\sim \sqrt{r-r_{1}}$ at large $r$. The part $V_{f 0}(\lambda)$ of the fermionic potential is given by eq. (2.30) and $a(\lambda)$ and $\kappa(\lambda)$ are given in (2.38). Notice that the deconfinement temperature $T_{d}$ always equals the temperature of the "standard" 1 st order transition $T_{h}$ in the holographic framework. The temperature of the chiral symmetry restoration $T_{\chi}$ can be either $T_{\text {end }}$ or $T_{h}$ depending on the order of the transitions, see examples below.

The result for the SB-normalised case is shown in figure 13. For $0<x_{f}<x_{\chi} \simeq 2.46$ we find that $T_{\text {end }}<T_{h}$, but $T_{\text {end }}$ is in the metastable branch of the solution. Thus the 

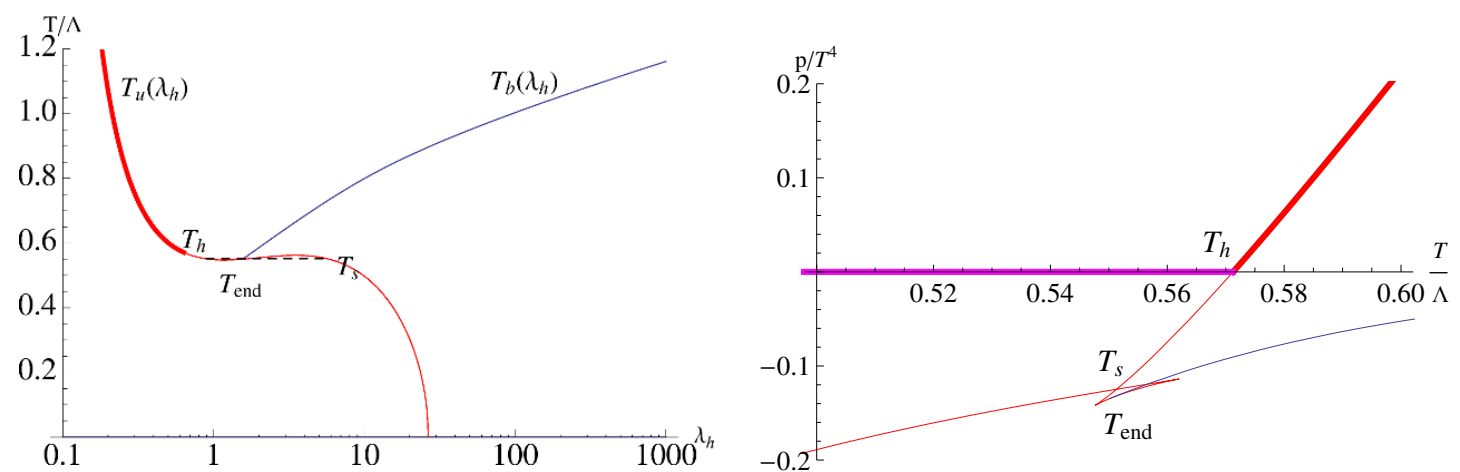

Figure 12. An example of a configuration where all but the crossover and hadronisation transitions $T_{\text {crossover }}, T_{h}$, are in the thermodynamically unstable region, in the initial stages of the approach to the IHQCD limit. The potential is II with $W_{0}=12 / 11$ and with $x_{f}=0.4$ Left: the temperature $T\left(\lambda_{h}\right)$. Note that everything to the right of the $T_{h}$ transition is in the unstable phase. Right: $p(T) / T^{4}$ in the region where the $T_{h}$ transition and the unstable $T_{\text {end }}$ and $T_{\mathrm{S}}$-transitions happen.

deconfinement and chirality transitions coincide here, $T_{d}=T_{\chi}=T_{h}$. In other words, if one could sufficiently supercool the system below $T_{h}$ in the high- $T$ chirally symmetric phase, the symmetry breaking transition could take place at $T_{\text {end }}<T_{h}$. In the thermodynamic limit there is no supercooling and only $T_{h}$ is seen.

Above $x_{f} \simeq 2.46$, the second order $T_{\text {end }}$ moves above $T_{h}$ and becomes stable, as seen in the bottom right plot of figure 13. Therefore, we first have a 1st order $T_{h}$ transition from the thermal gas solution to a chirally breaking black-hole phase, and then a 2nd order transition from the chirally broken low- $T$ phase to the chirally symmetric high- $T$ phase. In other words, $T_{\chi}>T_{d}$ with a 2 nd order chiral and 1st order deconfinement transition. For a more detailed view of the thermodynamics in this region at $x_{f}=3$, the reader is guided to the left panel of figure 24 where the chiral condensate as well as the energy and the pressure are plotted as functions of $T$. The chirally symmetric crossover transition $T_{\text {crossover }}$ is for all $x_{f}$, the highest temperature transition.

For $x_{f} \rightarrow x_{c}$ both $T_{\text {end }}$ and $T_{h}$ are expected to approach zero as specified by Miransky scaling. Numerical results are compatible with this.

When $x_{f} \rightarrow 0$ one would expect that the $T_{h}$ transition smoothly approaches the transition temperature of large $N_{c}$ hot Yang-Mills theory. Note, however, that strictly speaking the limit of YM theory demands $N_{f}=0$ and falls outside the Veneziano limit $N_{f} \rightarrow \infty$ of QCD. Thus it is not surprising that nontrivial metastable structures appear at $x_{f} \rightarrow 0$. What happens is that the curve $T=T_{u}\left(\lambda_{h}\right)$ of the chirally symmetric phase suddenly at $x_{f} \sim 0.2$ develops a local minimum similar to the one shown in red in figure 10 . Further evolution of this minimum is shown in figure 22. Associated with this there is a first order $T_{\mathrm{s}}$ transition in the metastable branch. It is so slightly below $T_{h}$ that it is not visibly separated in the bottom left plot of figure 13. As discussed in section 4.8, both $T_{h}$ and $T_{\mathrm{s}}$ approach the transition temperature of $\mathrm{YM}$ as $x_{f} \rightarrow 0 . T_{\text {end }}$ crosses above all of the other transitions for low $x_{f}$, but it is also in the metastable branch, see figure 12 for details. 

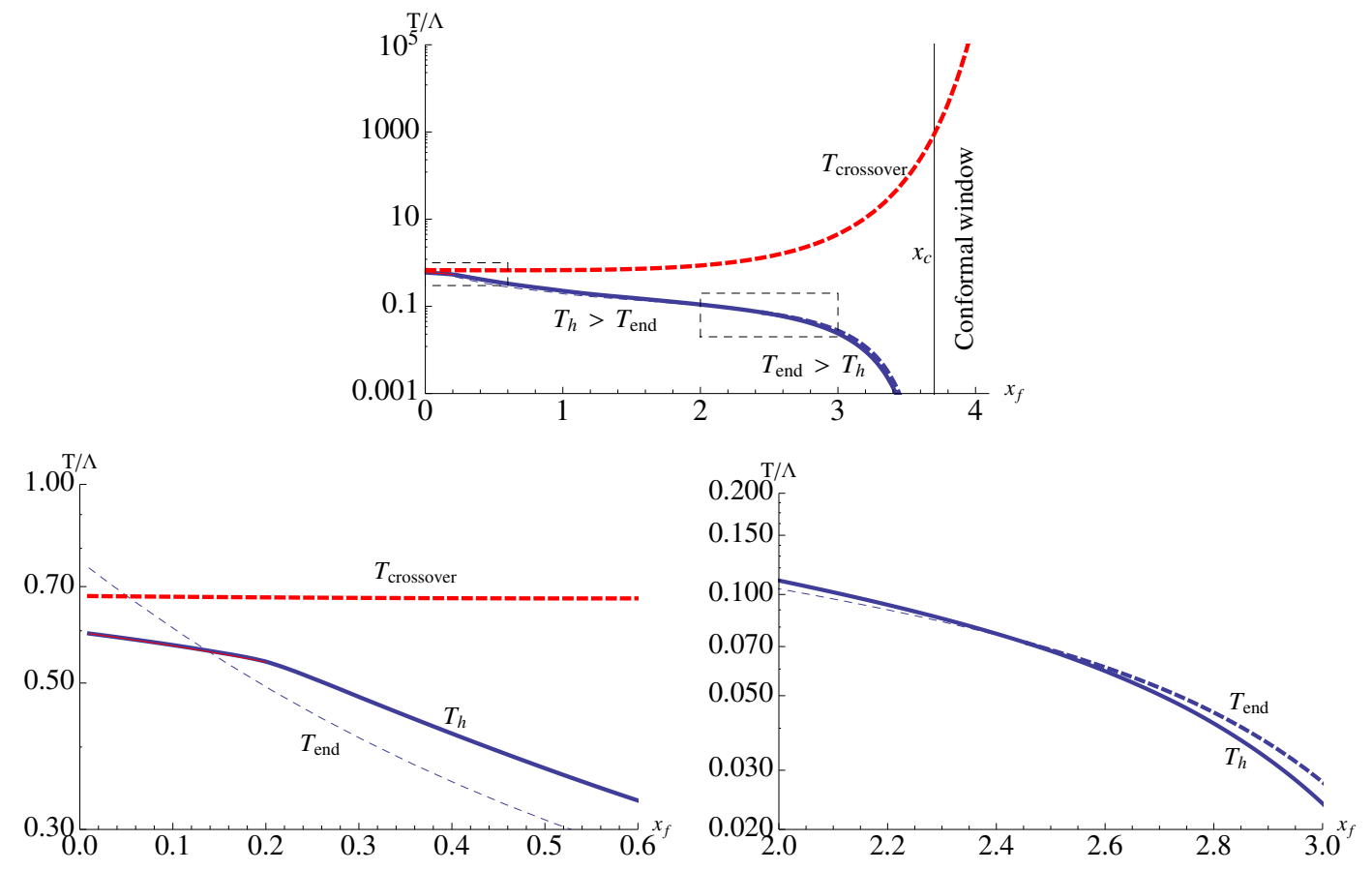

Figure 13. Upper: the phase diagram for potential II, $W_{0}$ Stefan-Boltzmann normalized, $x_{c}=3.70$. The dashed boxes show the regions detailed in the bottom two plots. In the bottom left plot $T_{s} \lesssim T_{h}$ at $x_{f} \lesssim 0.2$ is not visibly separated. For discussion, see text.

The phase diagram for potential II at $W_{0}=24 / 11$ is shown in figure 14 . The phase structure is qualitatively similar to the SB-normalized case. For $x_{f}<x_{\chi} \simeq 3.19$ the stable $T_{h}$ transition is the only one in the thermodynamic limit, with $T_{\text {end }}<T_{h}$ in the metastable branch of the solution. Thus again $T_{d}=T_{\chi}$. Above $x_{f} \simeq 3.19$, the second order $T_{\text {end }}$ moves above $T_{h}$ and becomes stable, see bottom right plot of figure 14. Thus we again have $T_{\chi}>T_{d}$ with a 2nd order chiral and 1st order deconfinement transition. The chirally symmetric crossover transition $T_{\text {crossover }}$ is for all $x_{f}$ the highest temperature stable transition, except between $x_{f} \sim 1$ to $x_{f} \sim 2.7$, where the interaction measure does not have a maximum and the crossover therefore does not exist.

Now $T_{s}$ which appears in the metastable branch slightly below $T_{h}$ in figure 10 (bottomleft) visibly separates from $T_{h}$. Again $T_{s}$ and $T_{h}$ approach the temperature of the YMtransition in the $x_{f} \rightarrow 0$-limit, as discussed in section 4.8. $T_{\text {end }}$ crosses above the $T_{h}$ transition for $x_{f}<0.34$, but it is also in the metastable branch, see figure 12 for details.

The phase diagram for potential II at $W_{0}=12 / 11$ is shown in figure 15 . The main difference with respect to the previous cases is that $T_{\text {end }}<T_{h}$ for all values of $x_{f}$, so the region with $T_{\chi}>T_{d}$ does not exist. Notice that $T_{\text {end }}$ is close to $T_{h}$ for $x_{f} \rightarrow x_{c}$ as seen from figure 15 (left). Because the region with small $x_{c}-x_{f}$ is numerically challenging, we do not have reliable data for $x_{f} \gtrsim 3.8$. However, nontrivial structure apart from the Miransky scaling, such as rapid changes in the ratios of the various temperatures, are not expected in this region (see discussion below in section 4.8). The chirally symmetric 

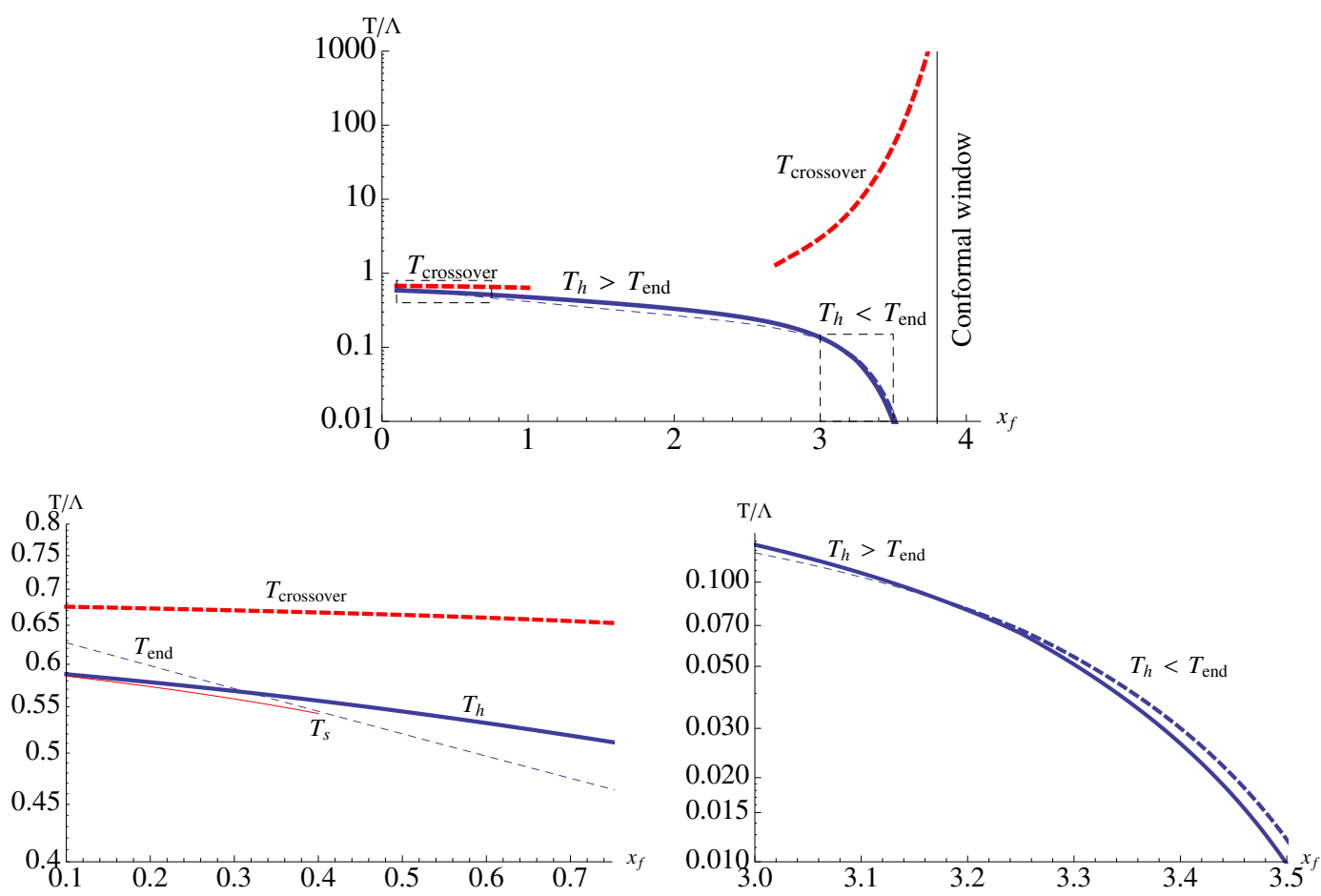

Figure 14. Upper: the phase diagram for potential II, $W_{0}=24 / 11, x_{c}=3.80$. The dashed boxes show the regions detailed in the bottom two plots. For discussion, see text.

crossover transition $T_{\text {crossover }}$ is the highest temperature stable transition where it exists. The next stable transition is everywhere $T_{h}$, and as already pointed out, $T_{\text {end }}$ is in the metastable branch of the solution. Details of further metastable structure at small $x_{f}$ are shown in the right hand plot. At $x_{f} \sim 0.25$, the first order $T_{\mathrm{s}}$ transition appears in the metastable branch slightly below $T_{h}$, see figure 10 . This transition develops into the YM transition in the $x_{f} \rightarrow 0$-limit. $T_{\text {end }}$ crosses above the $T_{h}$ transition, but it is also in the metastable branch, see figure 12 for details.

The phase diagram for potential II at $W_{0}=0$ is shown in figure 16. For all points shown, $T_{\text {end }}$ is below $T_{h}$ and in the metastable branch. The crossover exists when $x_{f} \gtrsim 3.6$ and again between $x_{f}=0$ to $\sim 0.7$. The close-up of the small $x_{f}$-region in the right hand plot shows the crossover and the hadronisation transition $T_{h}$, with the $T_{\text {end }}$ and $T_{s}$ transitions in the metastable branch. As a new feature the crossover also becomes metastable for $0.5 \lesssim x_{f} \lesssim 0.7$.

Finally, let us comment on the $x_{f}$ dependence of the transition temperature(s). For SB normalised $W_{0}$ or $W_{0}=24 / 11$ (figures 13 and 14), $T_{h}$ and $T_{\text {end }}$ decrease with increasing $x_{f}$, in qualitative agreement with estimates based on field theory [74-77]. Decreasing $W_{0}$ to $12 / 11$ (figure 15), however, the $x_{f}$ dependence becomes almost flat, and for $W_{0}=0$ (figure 16) the temperatures increase with $x_{f}$ up to $x_{f} \simeq 3.5$. Rather similar behavior with varying $W_{0}$ will be found for potentials I below, where the $x_{f}$-dependence is partially disturbed by the additional structure appearing at low $x_{f}$. 

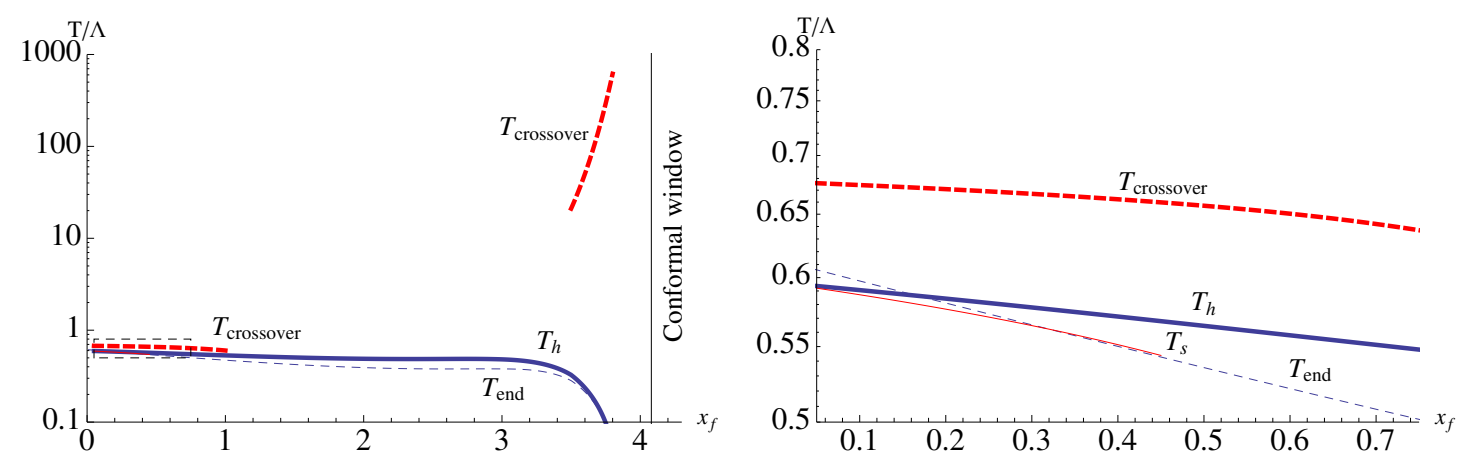

Figure 15. Left: the phase diagram for potential II at $W_{0}=12 / 11, x_{c}=4.08$. The dashed box shows the region detailed in the other plot. For discussion, see text.
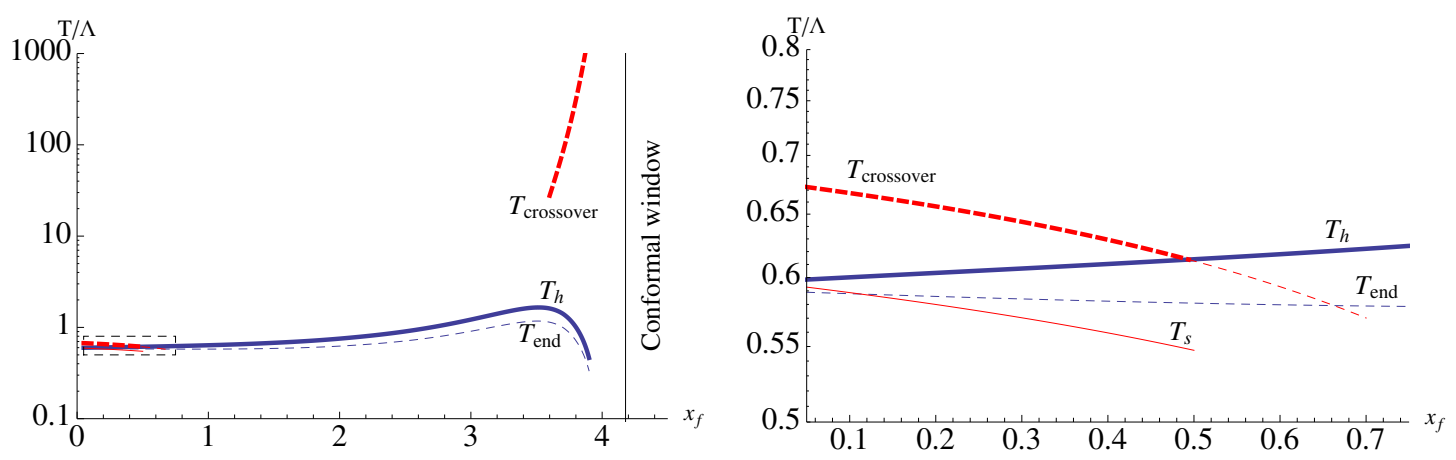

Figure 16. Left: the phase diagram for potential II at $W_{0}=0, x_{c}=4.18$. The dashed box shows the region detailed in the other plot. For discussion, see text. $T_{\text {crossover }}$ continues into the conformal window.

\subsection{Class-II Potentials $_{*}$}

In this section, we consider the phase diagram corresponding to the potential $\mathrm{II}_{*}$. Recall that the star subscript refers to the fact that the potential $V(\lambda)=V_{g}(\lambda)-x_{f} V_{0 f}(\lambda)$ has an extremum only for $x_{*}<x_{f}$, while for the cases discussed earlier such extremum exists for all values of $x_{f}$; see section 2.2.2 for detailed definitions.

The resulting $\left(x_{f}, T\right)$-phase diagram is shown in figure 17, the top panel shows how the phase diagram is derived at $x_{f}=2.5$. Starting at large $T$ one is in the tachyonless black hole phase (thick red curve). At $T_{h} \approx 0.8 \Lambda$ pressure goes to zero and the ground state is the thermal gas phase with $p=0$. If one could supercool further one would at $T_{\text {end }}$ meet the chirally broken tachyonic black hole phase. It has a higher free energy than the stable broken phase and therefore is unstable.

The main features are that the crossover exists only for small values of $x_{f}, x_{f} \lesssim 2$ where it nearly coincides with $T_{h}$, and again at larger values $x_{f} \gtrsim 3.5$, where it is clearly separated from $T_{h}$. The second order endpoint $T_{\text {end }}$ remains in the unstable phase for $x_{f} \leq x_{c}$. Below the conformal window, for values $2 \lesssim x_{f} \lesssim 4$ both $T_{h}$ and $T_{\text {end }}$ increase. They reach their maximum and finally start to decrease (as predicted by the Miransky scaling) only around $x_{f}=4$, very near the boundary of the conformal window. This suggests that the modification of the potential has the tendency to "squeeze" the walking region. 


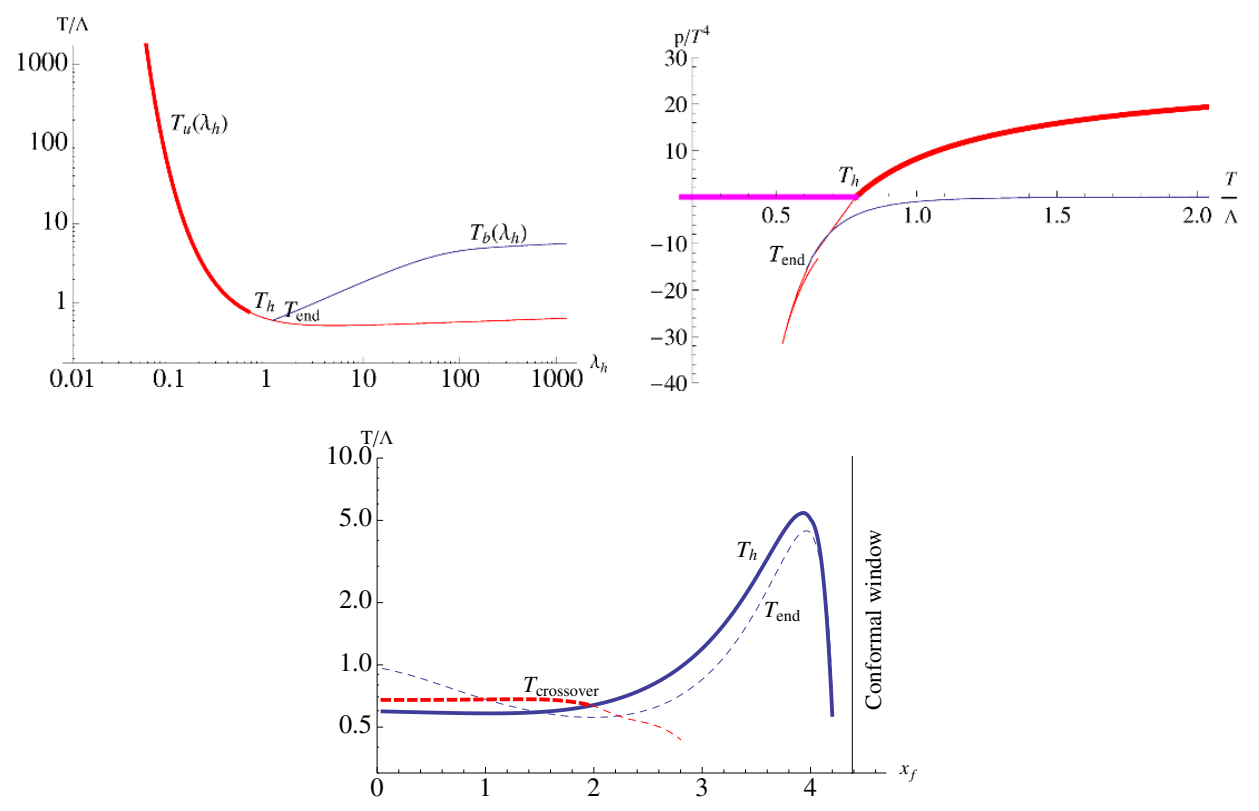

Figure 17. Phase diagram for potential $\mathrm{II}_{*}$ with $W_{0} \mathrm{SB}$ normalised (bottom). The top panel

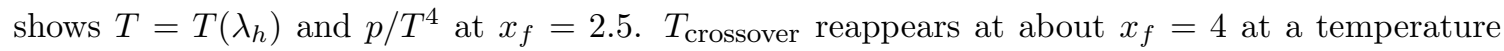
well above the range shown here, and continues into the conformal window.

\subsection{Class-I potentials}

For class I potentials figure 18 shows phase diagrams for $W_{0}=0,12 / 11,24 / 11$ and for the SB-normalised case. Recall that for these potentials the tachyon diverges exponentially in the IR. The choices of $a$ and $\kappa$ are given in eqs. (2.36). We also remind that transitions between stable phases are plotted as thick lines. Transitions plotted as thin lines can be seen only if the system is, e.g., supercooled, so that they are not there in the thermodynamic limit.

One can observe several characteristic features for varying $W_{0}$ :

- The first observation is the striking structure near $x_{f}=0$ which is observed at large $W_{0}$, i.e., for $W_{0}=24 / 11$ or SB normalized. The temperatures $T_{h}$ and $T_{\text {end }}$ drop rapidly with decreasing $x_{f}$ near $x_{f}=0$ and reach zero at a finite value of $x_{f}$. Below this critical value, all phases are chirally symmetric.

This behavior is related to the tachyon mass at the IR fixed point, shown in figure 3 . For PotI (the absolute value of) the squared tachyon mass is below the BF bound for low values of $x_{f}$. Therefore it is not guaranteed that a solution with zero quark mass and nontrivial tachyon profile exists (at any temperature) in this region. For large $W_{0}$ it actually turns out that the solution with $m_{q}=0$ and nontrivial tachyon profile does not exist for very low $x_{f}$, which explains the absence of chiral symmetry breaking. This implies that this potential is not describing a QCD-like theory. However, the applicability of PotI can be rescued by a simple logarithmic modification of $\kappa(\lambda)$, see section 4.6 and figure 20 . 
- The symmetric $\rightarrow$ symmetric transition $T_{s}$ becomes a stable transition when $W_{0}=$ 24/11 or SB normalized. For comparison, for PotII it was always in a metastable phase. This happens mostly in the region of very low $x_{f}$ where all phases are chirally symmetric so that $T_{h}$ and $T_{\text {end }}$ are absent. For $W_{0}=24 / 11$ we observe a region with $0.25 \lesssim x_{f} \lesssim 0.45$ where these transitions are also present. In this case the order of transitions is $T_{s}>T_{\text {end }}>T_{h}$, and chiral symmetry is broken in the middle one. For $W_{0}$ SB normalized we find instead a region with $0.2 \lesssim x_{f} \lesssim 0.5$ where only the crossover exists, so that the phase structure is similar to the conformal window.

- At large $x_{f} \gtrsim 3, W_{0}=12 / 11$, one observes the splitting of the 1st order line $T_{h}$ into two 1st order lines $T_{12}>T_{h}$. The order of the transitions is $T_{\text {end }}>T_{12}>T_{h}$, chiral symmetry is broken at the largest one, $T_{\text {end }}$. The holographic action therefore gives two consecutive 1st order transitions within the chirally-broken phase. It is an open issue what the nature of these transitions is. It is plausible that PotI at large $W_{0}$ is not related to QCD-like theories.

- The high temperature crossover exists over a larger and larger range when $W_{0}$ increases and ultimately appears at all $x_{f}$. This is the same tendency seen also for potentials in the II class.

\subsection{Class- $\mathrm{I}_{*}$ potentials}

Finally, we present the phase diagram corresponding to the potential $I_{*}$ in figure 19 . The striking difference between the phase diagram of the potential $\mathrm{I}_{*}$ in comparison with potential $\mathrm{II}_{*}$ considered earlier is that for small values of $x_{f} \lesssim 2$ there are no solutions with broken chiral symmetry, not even at low temperatures; all phase boundaries here are between chirally symmetric phases. There is $T_{h}$, but now it describes a chirally symmetric $\rightarrow$ symmetric transition. To illustrate this we show explicitly $T=T\left(\lambda_{h}\right)$ at $x_{f}=1$. It is very structureless, and has no solutions with nonzero tachyon. Thus the $\left(\lambda_{f}, T\right)$-diagram is qualitatively similar to the Yang-Mills case [33-35]. Only above $x_{f} \sim 2$ and below $x_{c}$ is chiral symmetry broken at low temperatures.

Otherwise the overall features are similar to those in the case of potential $\mathrm{II}_{*}$. For small values of $x_{f}, x_{f} \lesssim 2$, the crossover nearly coincides with $T_{h}$. The second order endpoint, $T_{\text {end }}$, is in the unstable branch for small values of $x_{f}$, but enters into the stable branch at $x \sim 4$. Below the conformal window, for values $2 \lesssim x_{f} \lesssim 4$ both $T_{h}$ and $T_{\text {end }}$ increase. They start to decrease only at $x_{f} \sim 4$, very near the boundary of the conformal window.

We have also studied the potentials $I_{*}$ for the case of fixed $W_{0}$ and found qualitatively similar results for the phase structure for $W_{0}=12 / 11,24 / 11$. For $W_{0}=0$ the problematic region without chiral symmetry breaking is absent, and the phase diagram is similar to PotII $_{*}$. This implies that, like Pot I, this type of potential is probably not applicable for QCD-like theories when $W_{0}$ is large.

\subsection{PotI with logarithmic correction to $\kappa(\lambda)$}

The function $\kappa(\lambda)$ in the action (2.2) represents the effects of going from the string frame (to which the derivation of the DBI action as the $\alpha^{\prime} \rightarrow 0$ limit of open strings leads) 

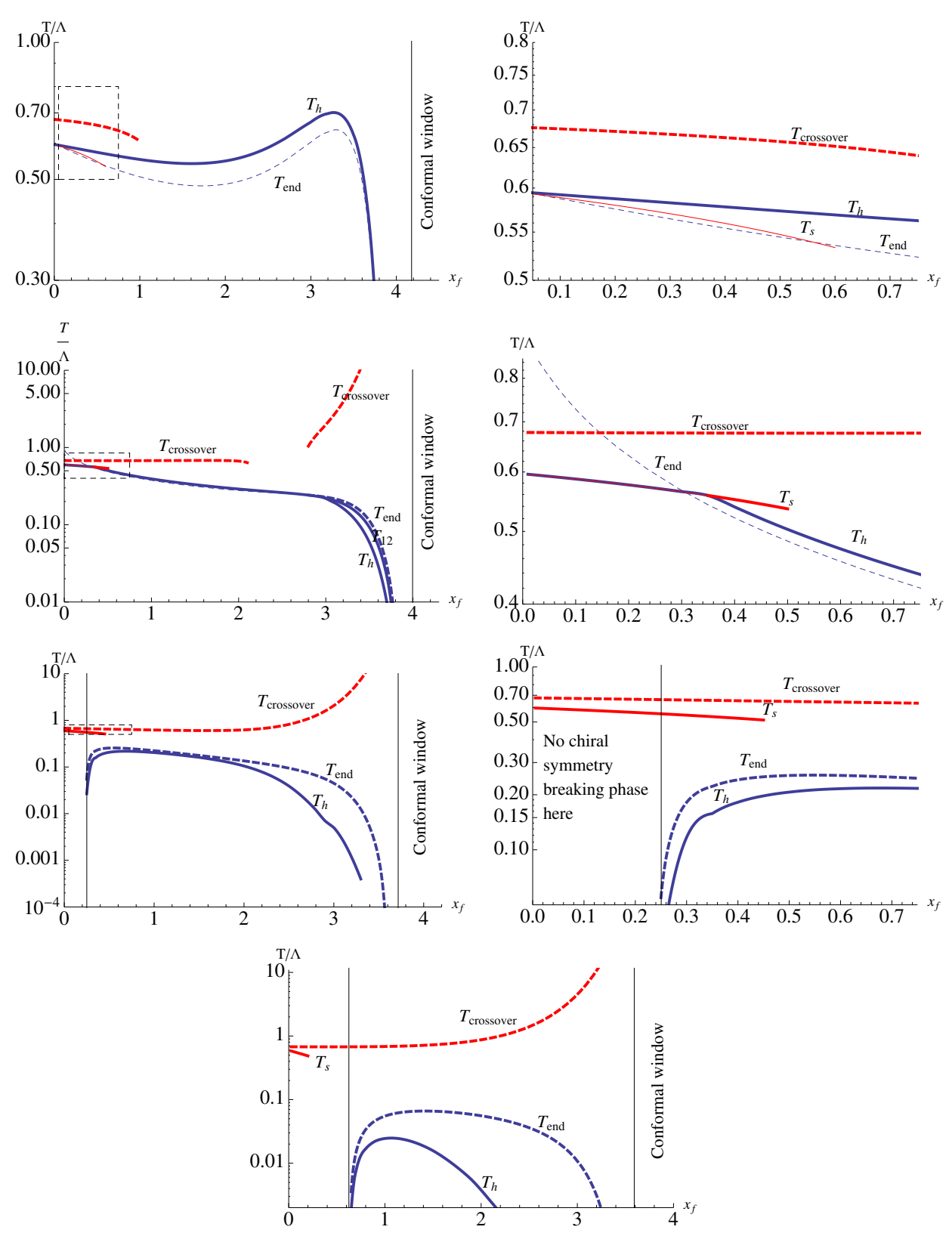

Figure 18. Phase diagrams for potential I. Top: $W_{0}=0$; Middle ones: $W_{0}=12 / 11,24 / 11$; Bottom: SB normalisation of $W_{0}$. A blow-up of the small $x_{f}$ region is shown at right separately for three top rows. The leftmost vertical line in the bottom three figures denotes the value of $x_{f}$ below which chiral symmetry breaking solutions do not exist.

and the Einstein frame (where the gravity dual is formulated). Extending the conformal transformation relating these to UV by $\lambda \rightarrow 1+\lambda$ one has, in terms of the metric functions, ${ }^{15}$

$$
\kappa(\lambda)=\frac{1}{(1+\lambda)^{4 / 3}}=\frac{b^{2}}{b_{s}^{2}}
$$

\footnotetext{
${ }^{15}$ Notice that we introduced additional constants in the formulas (2.36) and (2.38) in order to match with the perturbative anomalous dimensions in QCD.
} 

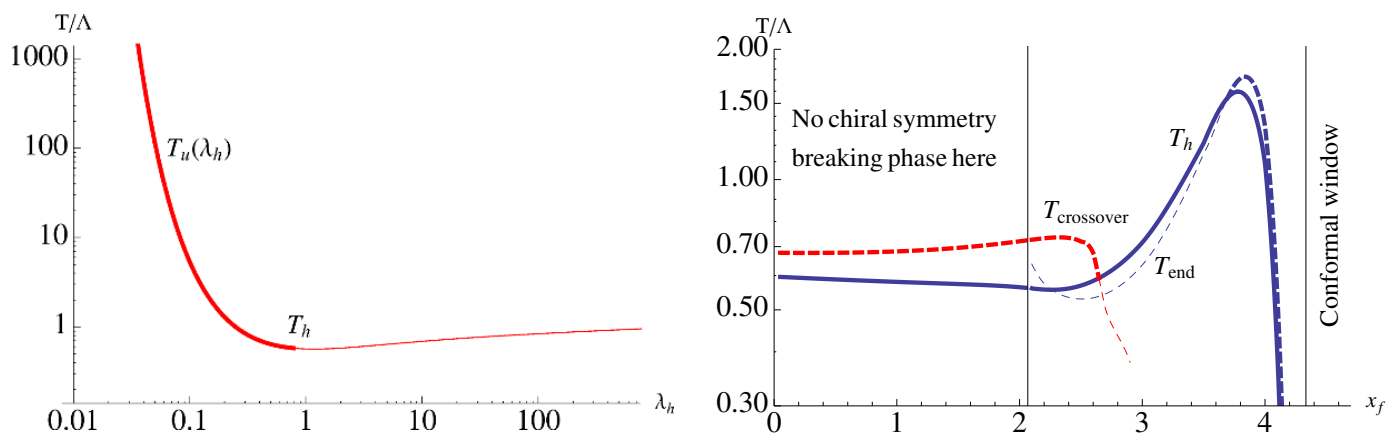

Figure 19. Phase diagram for potential $\mathrm{I}_{*}$ with $\mathrm{SB}$ normalisation of $W_{0}$. The left panel shows $T=T\left(\lambda_{h}\right)$ at $x_{f}=1$ : no tachyonic black hole!

where $b$ and $b_{s}$ are the metric factors in the Einstein and string frames, respectively.

The potential (2.27) carries the factor $\lambda^{4 / 3}$, but also the logarithmic factor $(\ln \lambda)^{1 / 2}$, which plays a quantitatively important role: for $(\ln \lambda)^{P}$ the excitation spectrum is $m \sim$ $n^{P}[30-32]$ and one wants the Regge-like spectrum, $P=1 / 2$. Also numerically $\ln \lambda$-effects are important, see figure 7 . To study these effects in $\kappa$ we use the parametrisation

$$
\kappa(\lambda)=\frac{[1+\ln (1+\lambda)]^{\bar{\mu}}}{\left[1+\frac{3}{4}\left(\frac{8}{9} b_{0}+1+\bar{\mu}\right) \lambda\right]^{4 / 3}} .
$$

There are constraints on this parametrisation from the UV and IR. First, to maintain the proper mass anomalous dimension equation (2.26) at small $\lambda, \bar{\mu}$ has to appear also in the denominator as shown in (4.2). Secondly, for $\bar{\mu}=0$ the tachyon grows exponentially in $r$ according to eq. (2.37). The effect of $\bar{\mu}$ on this comes from the change $b^{2} / \kappa=$ $\sqrt{\ln \lambda} /(\ln \lambda)^{\bar{\mu}} \sim r^{1-2 \bar{\mu}}$ (in the IR $r \sim \sqrt{\ln \lambda}$, see (B.4)). This effect propagates through the computation of the $r$ dependence which comes out to be $\tau(r) \sim \exp \left(C r^{1-2 \bar{\mu}}\right)$, indicating that $\bar{\mu}<1 / 2$.

The most interesting effect comes from evaluating the tachyon IR mass using (2.42). The result is shown in figure 20 , to be compared with figure 3 . The difficulty with PotI was that at small $x_{f}$ the curve in the left panel of figure 3 dropped below the BF bound. The reason for this is easy to see analytically by studying the $\lambda^{*} \rightarrow \infty$ limit of (2.42), which gives $-m_{\mathrm{IR}}^{2} \ell_{\mathrm{IR}}^{2} \sim\left(-\ln x_{f}\right)^{-1 / 2}$ in this case. For small $x_{f}, \lambda^{*}$ approaches infinity and obviously negative values of $\bar{\mu}$ increase the tachyon mass $-m_{\mathrm{IR}}^{2} \ell_{\mathrm{IR}}^{2}$, so that $-m_{\mathrm{IR}}^{2} \ell_{\mathrm{IR}}^{2} \sim\left(-\ln x_{f}\right)^{-\bar{\mu}-1 / 2}$ For $\bar{\mu}<-1 / 2$ it even grows without bounds as for PotII in figure 3. This is seen in figure 20.

As a consequence, the phase diagram for PotI with log-modified $\kappa$ does not suffer from the problems at small $x_{f}$ described earlier for PotI. The phase diagram computed for $\bar{\mu}=-\frac{1}{2}$ is shown in figure 20 and, in fact, resembles qualitatively those for PotII. This is very gratifying since PotI also leads to a Regge-like particle spectrum [70]. PotI with log-modified $\kappa(\lambda)$ (4.2) thus seems to be the gravity dual leading to the simplest thermodynamics in figure 8 and the expected Regge-like hadron spectrum. It is interesting that also PotII, a dual with spectrum of type $m \sim n$, also leads to the simple thermodynamics in figure 8. 

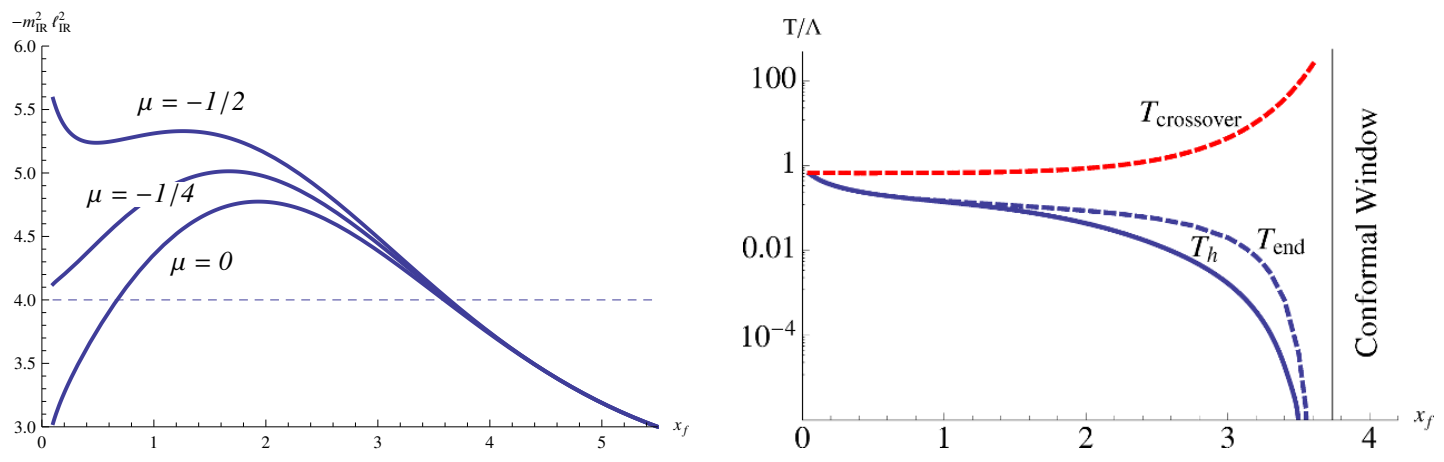

Figure 20. Left: the tachyon mass at $\lambda^{*}$ for PotI with $\log$-modified $\kappa(\lambda)$, evaluated using eqs. (2.42) and (4.2) for $\bar{\mu}=0,-\frac{1}{4},-\frac{1}{2}$. The $\bar{\mu}=0$ curve is the same as the red dotted curve in left panel of figure 3. Right: the phase diagram for PotI with SB-normalised $W_{0}$ with $\kappa(\lambda)$ given by (4.2) with $\bar{\mu}=-\frac{1}{2} \cdot T_{\text {end }}$ intersects $T_{h}$ at $x_{f}=x_{\chi}=0.72 . T_{\text {crossover }}$ is the same as in figure 18 , bottom.

\subsection{The conformal window}

A detailed picture of thermodynamics in the conformal window is shown in figure 21 . Here $p / T^{4}$, i.e. the effective number of degrees of freedom, is plotted for some values of $x_{f}>x_{c}$. At large $T$ it is normalised so that it approaches the SB limit (3.38) for any $x_{f}$. For $T$ approaching zero, $p / T^{4}$ approaches another constant, the value of which decreases when $x_{f}$ approaches $x_{c}$ from above. For all $x_{f}$, the vacuum phase has zero pressure, and at the limit $T \rightarrow 0$ there is a transition from the black hole to the thermal gas phase. When $x_{f}$ approaches the upper end of the conformal window 11/2, the behavior of the curves can be worked out analytically in perturbation theory [71] since the coupling then is small.

For the present potential the finite temperature transition between the low and high temperature phases inside the conformal window is a smooth crossover. Figure 21 also plots the interaction measure, the maximum of which defines the critical temperature for this crossover. Note that even if the transition here is smooth crossover, the transition can also be of 1st order in different theories [45]. What determines this behavior is the overall magnitude of the beta function. For illustration, consider the beta function of large $N_{f}$ QCD, $\beta=-b_{0} \lambda^{2}-b_{1} \lambda^{3}+\ldots$ The values of the coefficients behave as $b_{0} \sim \mathcal{O}(1) \ll$ $\left|b_{1}\right|$, while the results of [45] suggest $b_{0} \sim \mathcal{O}(10) \sim\left|b_{1}\right|$ for 1 st order phase transition. For the models we have considered here, we find that the nonperturbative beta function extracted from the gravity solution is small over the entire range $0 \leq \lambda \leq \lambda_{*}$ inside the conformal window.

The large temperature values appearing in figure 21 may appear somewhat surprising. However, they have a simple explanation. The region in which $p / T^{4}$ is nearly constant and approaching its large $T$ limit is the perturbative region $\lambda$ small. The conformal window is within $0<\lambda<\lambda^{*}\left(x_{c}\right)$ and the upper limit is always small, $\ll 1$, so that the conformal window is perturbative, down to $T=0$. From figure 2 one sees quantitatively how this holds even somewhat below the conformal window. To 1-loop $\log T=\exp \left(1 /\left(b_{0} \lambda\right)\right)$ and clearly for $b_{0} \rightarrow 0$ this grows fast. Somewhat more quantitatively, the beginning of the large $T$ region can be estimated by computing the value of $T$ for which the 2-loop correction 


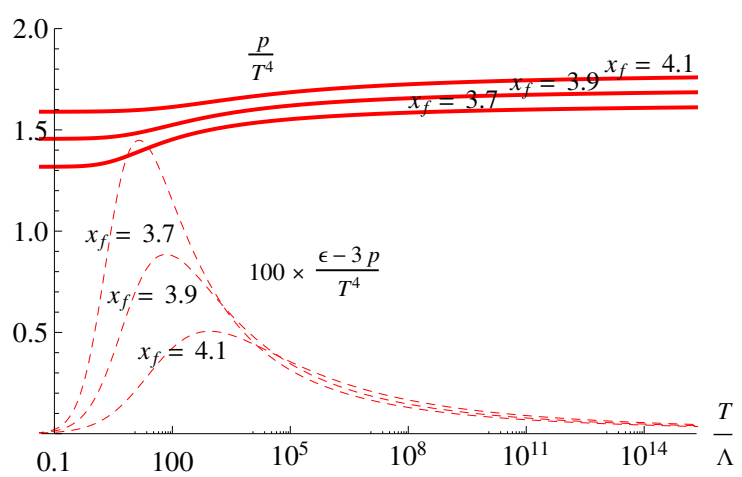

Figure 21. Thermodynamics for some values of $x_{f}$ within the conformal window, computed for PotI. Note that in the conformal window always $\tau=0$ and the functions $a(\lambda), \kappa(\lambda)$ do not affect the result.
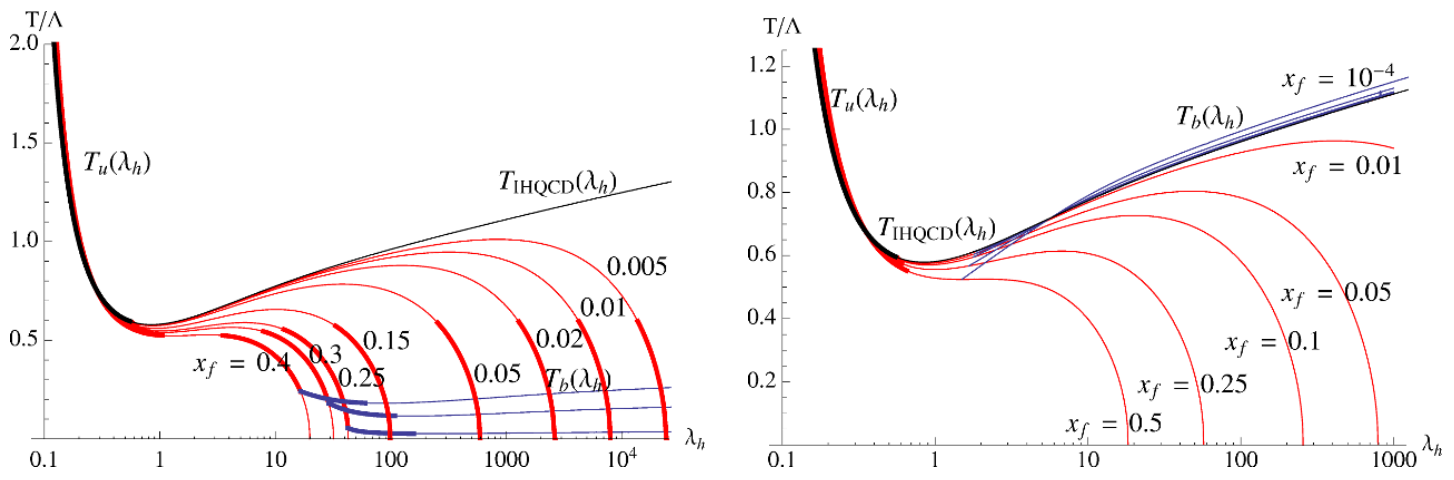

Figure 22. $T\left(\lambda_{h}\right)$ for various small values of $x_{f}$ and for potential I, $W_{0}=24 / 11$ (Left) or for potential II, $W_{0}=12 / 11$ (Right). The black curve is the IHQCD limit. The chirally unbroken $T_{u}\left(\lambda_{h}\right) \equiv T\left(\lambda_{h}, \tau=0\right)$ branch asymptotes to the IHQCD curve as $x_{f} \rightarrow 0$, for both potentials. The chirally broken $T_{b}\left(\lambda_{h}\right) \equiv T\left(\lambda_{h}, \tau_{h}\left(\lambda_{h}, m_{q}=0\right)\right)$ branches behave very differently for PotI and PotII. For PotI $T_{b}$ is absent at such low $x_{f}$ and all phases are chirally symmetric (see also figure 18). For PotII the curves $T_{b}$ follow very closely $T_{s}$ and, correspondingly, $T_{h} \approx T_{s}$ (see figure 15 ).

term in the perturbative expansion of $1 / \lambda(\mu)$ equals the 1-loop term. One finds that the 2-loop correction is smaller than the 1-loop term if $T>(2 \log T)^{\left|b_{1}\right| / b_{0}^{2}},\left|b_{1}\right| / b_{0}^{2}=$ $3\left|13 x_{f}-34\right| /\left(2\left(11-2 x_{f}\right)^{2}\right)$. This is always true for $T>1=\Lambda$ if $x_{f}<3.6$. However, for $x_{f}>3.6$ this gives a lower limit of $T$ which grows extremely fast when $x_{f}$ grows within the conformal window. $T_{\text {crossover }}$ is somewhat below the solution of this equation. Numerical values are in qualitative agreement with figure 21.

\subsection{The limits $x_{f} \rightarrow 0$ and $x_{f} \rightarrow x_{c}$}

The V-QCD models at $x_{f}=0$ are equivalent to an IHQCD model with potential $V_{g}$. One thus expects that the hadronisation transition $T_{h}$ will approach the 1st order deconfining transition of $\mathrm{SU}\left(N_{c}\right)$ YM theory ${ }^{16}$ when $x_{f} \rightarrow 0$. However, for PotI and large $W_{0}$ this

\footnotetext{
${ }^{16}$ Note that strictly speaking the limit of YM theory demands $N_{f}=0$ and falls outside the Veneziano limit $N_{f} \rightarrow \infty$ of QCD. This may explain the nontrivial structures observed at $x_{f} \rightarrow 0$.
} 
cannot be the case, since the hadronisation transition does not exist for very low $x_{f}$, as we pointed out above. Indeed we see from the phase diagrams of figure 18, that the symmetric transition $T_{s}$, which only exists for $x_{f} \lesssim 0.4$, is the precursor of the YM transition in this case.

Let us then discuss in detail what happens in the $x_{f} \rightarrow 0$ limit in the two cases and at finite temperature. Since thermodynamics is determined by the set of $T\left(\lambda_{h}\right)$ curves in figure 7, one should study how this configuration behaves when $x_{f} \rightarrow 0$. The $T\left(\lambda_{h}, \tau=0\right)$ curve (shown in red in figure 7) exists only for $\lambda<\lambda^{*}$ and, according to the definition (2.40) $\lambda^{*} \rightarrow \infty$ when $x_{f} \rightarrow 0$. In more detail, the limit is given by

$$
\lambda_{*}^{2 / 3}=\frac{8}{W_{0}+\frac{20568}{4619}} \frac{1}{x_{f}} \sqrt{\ln \lambda_{*}}, \quad V\left(\lambda_{*}\right)=\frac{18476}{729} \lambda_{*}^{4 / 3} \sqrt{\ln \lambda_{*}} .
$$

where the equation on the left determines $\lambda_{*}$ while the equality on the right expresses $V\left(\lambda_{*}\right)$ as a function of $\lambda_{*}$. Eq. (4.3) is valid both for potentials I and II. The red curves of figure 7 , therefore, stretch to the right when $x_{f} \rightarrow 0$. Quantitatively what happens is shown in figure 22 and one sees that they approach the $T\left(\lambda_{h}\right)$ curve of IHQCD when $x_{f} \rightarrow 0$. This is as expected since only $V_{g}$ remains in the limit. It is thus obvious that $T_{s}$ approaches the transition temperature of IHQCD as $x_{f} \rightarrow 0$ (but it may be a transition between two metastable phases rather than a physical transition).

To find the relative behavior of $T_{s}$ and $T_{h}$ one needs the asymptotic properties of the curves $T_{b}\left(\lambda_{h}\right) \equiv T\left(\lambda_{h}, \tau_{h}\left(\lambda_{h}, m_{q}=0\right)\right.$ ) (shown in blue in figure 7 ) which only exist for $\lambda>\lambda_{\text {end }}$. Here PotI and PotII behave in considerably different ways, as is already seen from figures 15 and 18 .

The crucial difference between PotI (at large $W_{0}$ ) and PotII comes from the fact that for PotI the value of $\lambda_{\text {end }}$ (the endpoint of the blue $T_{b}\left(\lambda_{h}\right)$ curves in figure 22) grows rapidly when $x_{f}$ decreases, while for PotII $\lambda_{\text {end }}$ remains almost constant. Since always $T_{u}\left(\lambda_{\text {end }}\right)=T_{b}\left(\lambda_{\text {end }}\right)$ and $T_{u}\left(\lambda_{h}\right)$ decreases rapidly at large $\lambda_{h}$, also the temperature $T_{\text {end }}$ becomes small at small $x_{f}$ for PotI. This drives the whole curve $T_{b}\left(\lambda_{h}\right)$ towards zero and since $T_{h}$ is determined by integration along $T_{b}\left(\lambda_{h}\right)$ also $T_{h} \rightarrow 0$. Finally $\lambda_{\text {end }}$ ceases to exist when $x_{f}$ goes below a critical value $\sim 0.25$, the temperatures $T_{\text {end }}$ and $T_{h}$ reach zero, and the low temperature chiral symmetry breaking phase disappears.

For PotII $T_{b}\left(\lambda_{h}\right)$ follows very closely $T_{\mathrm{IHQCD}}$ above it (figure 22 ) and it is thus natural that $T_{h} \gtrsim T_{s}$ and that they approach the same limit. $T_{s}$ is actually metastable (figure 15).

One can also illustrate the connection of the behavior of $\lambda_{\text {end }}$ to the BF bound of the tachyon (figure 3) by analyzing the linearized tachyon equation motion as discussed in appendix D.

In the limit of $x_{f} \rightarrow x_{c}$ one expects that all dimensionful quantities sensitive to the IR vanish as specified by Miransky scaling (1.8). All our numerical results are compatible with this, but conclusive numerical verification would require extensive further work. Analytic arguments supporting the scaling, similar to those presented in section 10 of [26], can also be constructed in the finite temperature case. We shall here, however, only briefly comment on the scaling as well as the overall behavior of the solutions as $x_{f} \rightarrow x_{c}$ from below. 


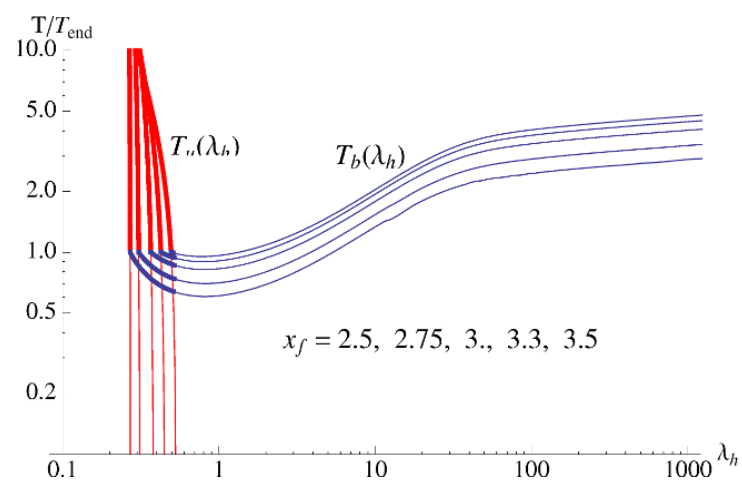

Figure 23. The temperatures $T_{u}\left(\lambda_{h}\right), T_{b}\left(\lambda_{h}\right)$ of the two black hole branches, unbroken and broken, scaled to the temperature $T_{\text {end }}$ for PotII, $W_{0}$ SB normalized. The values of $x_{f}$ from top to bottom are $2.5,2.75,3,3.3$, and 3.5. Compare to figures 9 and 13 .

We start with the case of zero temperature which is simpler. For $x_{f}<x_{c}$ the dominant background is the one with nontrivial tachyon, and chiral symmetry is broken. As $x_{f} \rightarrow x_{c}$ the solution comes closer and closer to the fixed point, and the near conformal region grows. As it turns out, the pieces for $\lambda>\lambda_{*}$ and $\lambda<\lambda_{*}$ approach separately fixed solutions in this limit, which do not talk to each other. ${ }^{17}$ Thus any observable which can be expressed only in terms of either the UV or the IR solution approaches a fixed value in the $x_{f} \rightarrow x_{c}$ limit. The ratio of the characteristic scales of the two pieces diverge as specified by the Miransky scaling factor of eq. (1.8).

It is hard to find simple examples of such observables at zero temperature, but as it turns out, at finite temperature there are plenty. However, the analysis of the limit is more involved, since we have the additional parameter $\lambda_{h}$ we can be either in the UV $\left(<\lambda_{*}\right)$ or in the IR $\left(>\lambda_{*}\right)$ regions. The curve which determines the main features of the thermodynamics is $T_{b}\left(\lambda_{h}\right)$, which lies mostly in the IR region. Its endpoint $\lambda_{\text {end }}$ is however smaller than $\lambda_{*}$. When $\lambda_{h}>\lambda_{*}$ we expect that the background solution breaks into two parts similarly as for $T=0$ in the limit $x_{f} \rightarrow x_{c}$, and the temperature is determined solely by the IR piece. ${ }^{18}$ In the limit $x_{f} \rightarrow x_{c}$ we find ${ }^{19}$ that $\lambda_{\text {end }} \rightarrow \lambda_{*}$ from below. Therefore the whole $T_{b}\left(\lambda_{h}\right)$ curve is in the IR region in the strict $x_{f} \rightarrow x_{c}$ limit, and it is plausible that it takes a fixed shape.

This behavior is supported by the numerical study of figure 23, where we plot the temperatures of the two black hole branches as functions of $\lambda_{h}$ for PotII with $W_{0}$ SB

\footnotetext{
${ }^{17}$ More precisely, keeping fixed the scale $\Lambda_{\text {IR }}$ defined by the IR expansions, the background approaches pointwise a "IR" limiting solution which flows from the good IR singularity to the fixed point at $\lambda=\lambda_{*}$. This solution which approaches $\lambda_{*}$ from the "wrong" side is possible due to the presence of the tachyon. Keeping the UV scale $\Lambda_{\mathrm{UV}}$ fixed instead, the "UV" limiting solution is the one that flows from the IR fixed point at $\lambda=\lambda_{*}$ to the standard UV fixed point at $\lambda=0$ with zero tachyon.

${ }^{18}$ There is a subtlety here as comparing temperatures at different $\lambda_{h}$ requires fixing the units of energy, which we do by calculating $\Lambda_{\mathrm{UV}}$. However for $\lambda_{h}>\lambda_{*}$ the UV part of the solution, and hence practically the units of energy, become independent of $\lambda_{h}$ in the limit $x_{f} \rightarrow x_{c}$. Thus we could equally well define the units in terms of the behavior of the IR piece of the solution near $\lambda=\lambda_{*}$.

${ }^{19}$ This is observed numerically, and can be understood by studying the violation of the BF bound in the spirit of appendix D.
} 

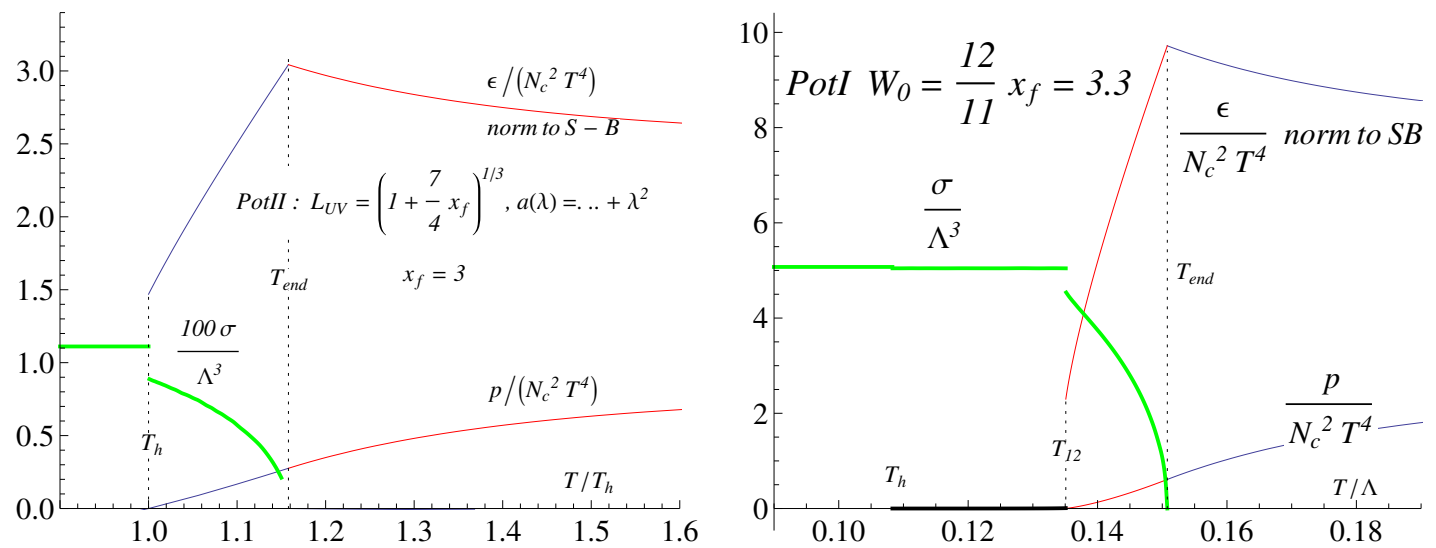

Figure 24. Examples of equation of state with $\bar{q} q$ condensate. Left: type II SB-normalised potentials at $x_{f}=3$ (compare figures 9 and 13). Note the scaling of $\sigma$ by a factor 100 . Right: type I potentials with $W_{0}=12 / 11$ and $x_{f}=3.3$ (compare figure 18 , middle panel).

normalized. The $x_{f}$-dependence of the curve $T_{b}\left(\lambda_{h}\right)$ is, up to the overall normalization, so small for $\lambda_{h} \gg \lambda_{*}$ that it cannot be resolved from the plot even at relatively low $x_{f} \lesssim 3$. The main effect with increasing $x_{f}$ is that the $T_{b}\left(\lambda_{h}\right)$ curve is visible down to lower and lower $\lambda_{h}$ as $\lambda_{*}$ decreases slowly, while the shape of the curve remains fixed. The curve $T_{u}\left(\lambda_{h}\right)$ approaches a vertical line when scaled to $T_{\text {end }}$, reflecting the fact that $\lambda_{\text {end }} \rightarrow \lambda_{*}$.

The values of all the critical temperatures (except $T_{\text {crossover }}$ ), as well as all thermodynamics up to the transitions, are determined by $T_{b}\left(\lambda_{h}\right)$ as $x_{f} \rightarrow x_{c}$. Therefore, we expect that the thermodynamics "freezes" in this limit, in the sense that all ratios of the critical temperatures approach fixed values. Moreover, the parts of the thermodynamical functions which are determined by the IR solutions, are expected to have well defined limits. While we have not proven these statements, they are strongly supported by the numerical study of figure 23. Notice however, that our data only extends up to $x_{f}=3.5$ which is still well below the critical value $x_{c} \simeq 3.70$. Therefore we cannot exclude the possibility that something drastic happens for $x_{f}$ even closer to $x_{c}$.

\section{The chiral condensate}

In principle, it should be straightforward to extract the chiral condensate $\propto \sigma$ from the tachyon solution in the UV

$$
\tau(r) \sim \sigma r^{3}(-\ln (\Lambda r))^{3 /\left(2 b_{0}\right)}, \quad r \rightarrow 0
$$

as the quark mass is set to zero. However, in this model the task is actually very demanding due to the logarithmic corrections (i.e., the running of the condensate) and the fact that the numerical solutions have a tiny residual quark mass due to limited numerical precision. These issues and their resolution are discussed in appendix E.

Examples of the correlation of $\sigma$ with that of the free energy are shown figure 24. One expects that $\sigma$ jumps in a 1st order transition. The first case is thermodynamics for SBnormalised type II potentials at $x_{f}=3$, also studied in figures 9 and 13 . Cooling from large 

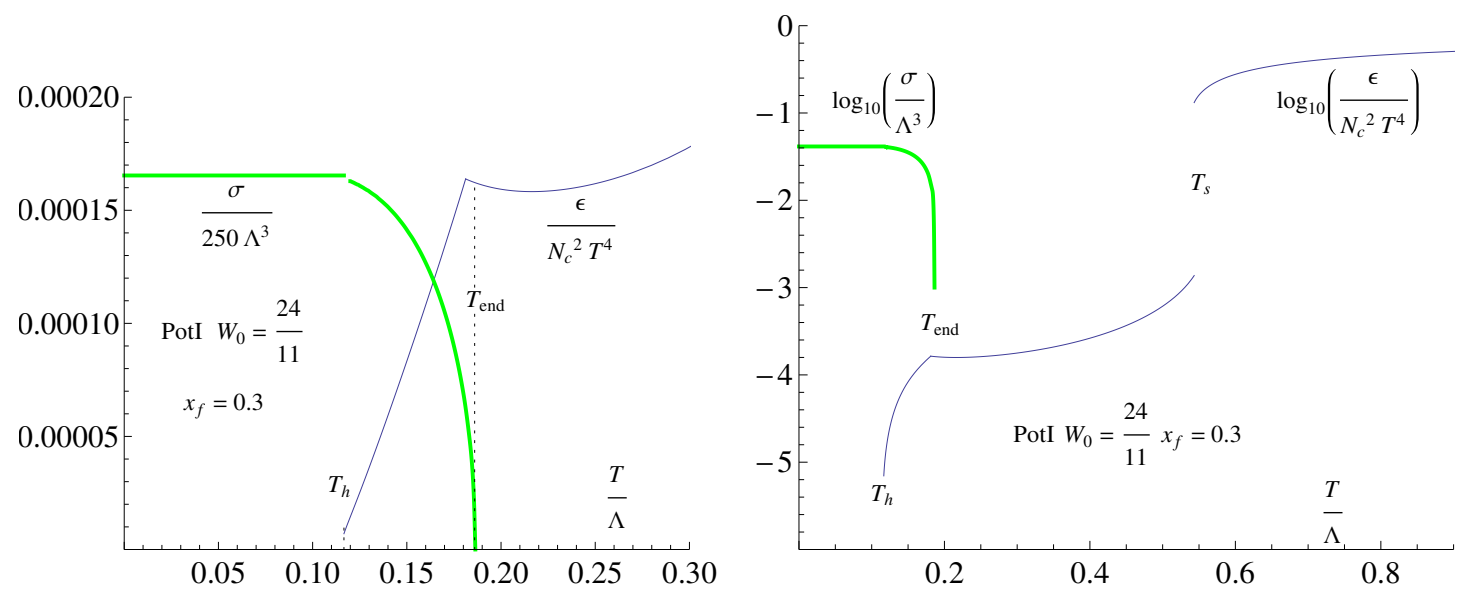

Figure 25. The condensate for the Pot I, $W_{0}=\frac{24}{11}$ phase diagram in the right hand column of figure 18 at $x_{f}=0.3$ in linear (Left) and $\log$ (Right) scale. Note the scaling of $\sigma$ by a factor $1 / 250$ in the left panel. The right panel shows all the three transitions in figure 18.

$T$ chiral symmetry first breaks at the 2 nd order transition $T_{\text {end }}$ and the condensate starts from zero and increases with further cooling. At $T_{h}$ the system experiences a 1 st order transition and $\sigma$ jumps the amount shown in the figure. Below that $\sigma$ remains constant in the present models, which does not describe the thermodynamics of the low $T$ phase. In that the degrees of freedom are $N_{f}^{2}$ massless Goldstone bosons.

A second example is thermodynamics with condensate of Type I potentials with $W_{0}=$ $12 / 11$ and $x_{f}=3.3$. This case is special in that in it the 1 st order line $T_{h}$ splits in two 1 st order transitions at $T_{h}, T_{12}$ if $x_{f} \gtrsim 2.8$, as shown explicitly in figure 18, middle panel. Again the highest temperature transition is a 2 nd order one at $T_{\text {end }}$, at which the condensate starts to grow when the system is cooled. The condensate grows up to the value $\sigma=4.537 \Lambda^{3}$. Then there is a 1 st order transition at $T_{12}$ with a jump in $\sigma$ and latent heat:

$$
\frac{\Delta \sigma}{\Lambda^{3}}=0.508, \quad \frac{\Delta \epsilon}{T_{12}^{4}}=2.29
$$

and finally a very weak transition at $T_{h}$ with the value

$$
\frac{\Delta \epsilon}{T_{h}^{4}}=1.03 \cdot 10^{-7}
$$

It is clear that the value of $\sigma$ also jumps at the latter transition, but the size of the jump is so small that we could not extract it reliably from the numerics.

As a third example, consider the case Pot I, $W_{0}=\frac{24}{11}$ at $x_{f}=0.3$, which is very special in that $T_{h}$ is very small and it is $T_{s}$ which dominates, as is seen in the right hand column of figure 18. The magnitudes vary so much that all the transitions can be presented only on log scale, see figure 25 .

At the 2 nd order transitions the condensate goes to zero continuously as the temperature approaches $T_{\text {end }}$ from below. The curves in figures 24 and 25 seem to be compatible with the standard expectation $\sigma \sim \sqrt{T_{\text {end }}-T}$. We study this more precisely in figure 26 


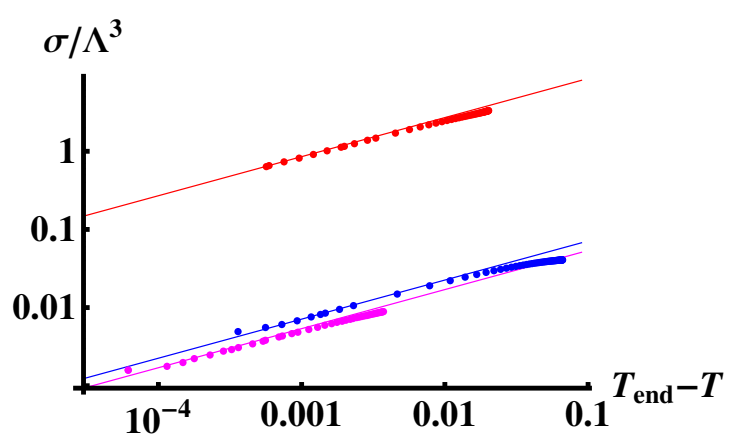

Figure 26. The condensate as a function of $T_{\text {end }}-T$ in $\log$-log scale. The dots are our data, extracted from the background solutions, and the lines are fits having the form $\sigma=C \sqrt{T_{\text {end }}-T}$. The choices of potentials are, from top to bottom: PotI with $x_{f}=3.5$ and $W_{0}=12 / 11$ (red); PotI with $x_{f}=0.3$ and $W_{0}=24 / 11$ (blue); and PotII with $x_{f}=3$ and SB normalized $W_{0}$ (magenta).

where we plot our data for the condensate for $T$ close to $T_{\text {end }}$ in the $\log$-log scale for various choices of the potentials. The data are compared to the lines $\sigma=C \sqrt{T_{\text {end }}-T}$, with the coefficients $C$ chosen such that the lines overlap with the tails of the data at small $T_{\text {end }}-T$. It is convincing that $1 / 2$ is indeed the correct exponent.

\section{Nonzero quark mass and thermodynamics}

We have so far mainly discussed the case of vanishing quark mass, for which chiral symmetry may hold. Effects of nonzero quark mass have been mentioned in subsection 3.3.2 and described in figures 6 and 7. They follow from the fact that tachyonfree black hole solutions do not exist, as a reflection of the disappearance of chiral symmetry. However, numerically the tachyonic small- $m_{q}$ solutions are very close to the zero mass tachyonfree solutions in the UV at small $\lambda$, as is seen by comparing left and right panels of figure 7 . Thus chiral symmetry is always broken even in the high- $T$ phase, but quantitative effects are small at large $T$.

The effects of small nonzero $m_{q}$ are shown quantitatively in figure 27 and can be summarised as follows:

- The main effect follows from the fact that for nonzero $m_{q}$ even the high $T$ phase is chirally broken. The curve marked $m_{q}=0$ is the same $T_{h}\left(x_{f}\right)$ as that in figure 13 . For this case the phase at $T>T_{h}$ is chirally symmetric and chiral symmetry is broken when $T$ decreases below $T_{h}$. For nonzero $m_{q}$ also the phase at $T>T_{h}$ is chirally broken and the effective order parameter of the transition is the jump in entropy or energy density. There is also a jump in the condensate, but the condensate is nonzero also for $T>T_{h}\left(x_{f}\right)$.

- For $x_{f}$ clearly below $x_{c}$ the effects of small $m_{q}$ on the phase diagram are small. Particularly interesting is the pattern of approach towards $m_{q}=0$. The smaller $m_{q}$, the higher is the value of $x_{f}$ where the curves start deviating significantly. 


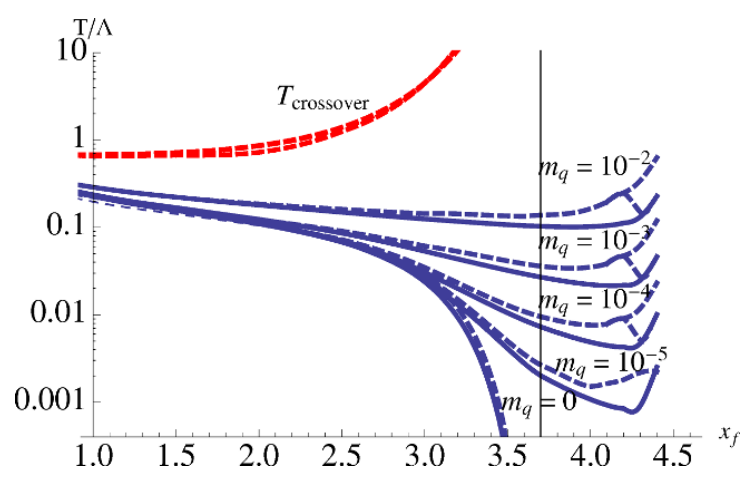

Figure 27. The behavior of $T_{h}\left(x_{f}\right)$ (in units of $1 / \mathcal{L}_{\mathrm{UV}}$, over the $x_{f}$ range in the figure this unit changes by about 30\%) for $m_{q}=0$ and for small values of $m_{q}$. For $m_{q}=0$ the dashed line shows the true 2 nd order chiral symmetry breaking transition. For nonzero $m_{q}$ the line shows the position of one maximum of the interaction measure, a second one gives the usual $T_{\text {crossover (which is almost }}$ independent of $m_{q}$ ).

- The 2nd order transition at $T_{\text {end }}$ becomes a continuous one. This is obvious from figure 7 , there is no discontinuity near $T_{\text {end }}$. However, a remnant of the genuine transition is a maximum of interaction measure, also plotted in figure 27.

- At large $x_{f}$ the conformal window and Miransky scaling disappear. For $m_{q}=0$ the transition temperature $T_{h}$ vanishes when $x_{c}-x_{f} \rightarrow 0$ as dictated by Miransky scaling. The smallest nonzero mass destroys this effect and $T_{h}$ curves upwards towards larger values.

- The effect of nonzero mass could also be seen by plotting the beta function for values of $x_{f}$ within the conformal window. For $m_{q}=0$ only the tachyonless solutions matter and they extend only up to $\lambda^{*}$ in figure 7 . The beta function $\beta(\lambda)$ only exists for $\lambda<\lambda^{*}$ and $\beta\left(\lambda^{*}\right)=0$ at the IR fixed point $\lambda^{*}$. For $m_{q}>0$ the beta function comes close to $\lambda^{*}$ but continues past it to larger values of $\lambda$.

\section{Outlook}

In this paper, we have used bottom-up holography to study the thermodynamics of models that are in the universality class of QCD with massless quarks in the Veneziano limit, (large $N_{c}$ and $N_{f}$ but fixed $\left.x_{f}=N_{f} / N_{c}\right)$.

The temperature dependence of the pressure $p(T)$ and the condensate $\sigma(T)=\langle\bar{q} q\rangle(T)$ was computed at various $x_{f}$ up to the loss of asymptotic freedom at $x_{f}=11 / 2$ with a conformal window appearing at $x_{c}<x_{f}<11 / 2, x_{c} \approx 4$. The singularities of these quantities define the phase diagram of the system.

One expects that the system has two phases, a low temperature phase with broken chiral symmetry and a chirally symmetric high $T$ phase. The simplest alternative is that these are separated by a 1 st order transition at some $T=T\left(x_{f}\right)$, see figure 8 . In holography, the high $T$ phase is a metric with a black hole and a new feature appears: this phase 
can be either chirally symmetric (no tachyon) or chirally broken (nonzero tachyon). The same doubling applies to the low $T$ thermal gas phase. The phase structure can be correspondingly more complicated. For given gluonic and fermionic potentials of the V-QCD action the thermodynamics is fixed and calculable.

A typical prediction of the model is that there indeed is a 1st order line for $x_{f}$ from 0 up to some value $\leq x_{c}$. In holography this is a transition at some $T=T_{h}\left(x_{f}\right)$ between a non-tachyonic black hole metric and a thermal gas metric with a tachyon. In field theory language one would say that at this temperature there is deconfinement and chiral symmetry breaking with coinciding deconfinement and chiral temperatures, $T_{d}=T_{\chi}$. However, a new interesting feature is that at larger $x_{f}$ this 1st order line can split in two: first at higher $T$ chiral symmetry is broken in a 2nd order transition, then at a lower $T$ there is a 1st order deconfinement transition (see, e.g., figure 13), $T_{\chi}>T_{d}$.

A particular feature of the phase diagram is that with $x_{f}$ approaching $x_{c}$ all transition temperatures, as all mass scales, decrease as specified by Miransky scaling. Associated with this approach is quasiconformality and walking. At very large $T$ there is always a

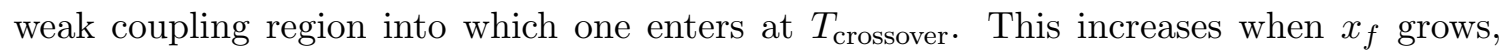
behaves regularly at $x_{c}$ and continues further into the conformal window increasing faster and faster (see again figure 13).

Detailed predictions of the model depend on the gluonic and fermionic scalar potentials. There are several physical constraints in deriving them, but they are not uniquely determined. One crucial constraint is missing: in contrast to the application of this model to hot $\mathrm{SU}\left(N_{c}\right)$ theory [33-35], there is no $4 \mathrm{~d}$ lattice data in the large $N_{c}, N_{f}$ limit. Thus one is genuinely making predictions and we find that also phase diagrams deviating rather drastically from the above baseline prediction follow from holography. Particularly striking examples are shown in figure 18. In these, the chirally symmetric phase can extend all the way down to zero temperature.

Although we do not expect this to happen in QCD, it may happen in other large Ntheories. The last diagram of figure 18 has a structure that is very reminiscent of the phase diagram of high- $\mathrm{T}_{c}$ cuprates, although here we are at zero charge density. In particular the intermediate dome-like structure with chiral symmetry breaking corresponds to the superconducting dome [78] in the cuprate diagram.

One may also note that the models predict a very rich structure of metastable states.

There are many directions in which the model could be further sharpened and developed:

- All the computations are numerically very demanding and much further work would be useful. This holds, in particular, for the approach to the conformal window, $x_{f} \rightarrow x_{c}$, and for computations of the condensate.

- Effects of non-zero quark mass on the thermodynamics should be studied beyond the discussion in section 6 .

- The extension to $x_{f 1}$ flavors of mass $m_{1}$, etc., is possible but requires a non-abelian version of the Sen action. This is in principle possible to deal with, although we have much less information from string theory on the details of such an action. 
- It would be very interesting to accumulate lattice Monte Carlo data in the Veneziano limit. For pure $\mathrm{SU}\left(N_{c}\right)$ theory already $N_{c}=3$ is very close to $N_{c}=\infty[73,79]$. One might thus see that, e.g., $N_{f}=4,8,12$ at $N_{c}=3$ would already give useful information.

- A chemical potential for baryons should be included. This necessitates the inclusion of a bulk $\mathrm{U}(1)$ baryon vector field $A_{\mu}$. It would be particularly interesting to know the fate of the $T_{\chi}>T_{d}$ phase at large $x_{f}$ and $\mu$.

\section{Acknowledgments}

We thank Ken Intriligator, Costas Kounnas, Vasilis Niarchos, Marco Panero, Gabriele Veneziano, for discussions. This work was in part supported by grants FP7-REGPOT2008-1-CreteHEPCosmo-228644, PERG07-GA-2010-268246, PIEF-GA-2011-300984, the EU program "Thales" ESF/NSRF 2007-2013, and by the European Science Foundation "Holograv" (Holographic methods for strongly coupled systems) network. It has also been co-financed by the European Union (European Social Fund, ESF) and Greek national funds through the Operational Program "Education and Lifelong Learning" of the National Strategic Reference Framework (NSRF) under "Funding of proposals that have received a positive evaluation in the 3rd and 4th Call of ERC Grant Schemes". TA thanks the Vaisala foundation for financial support.

\section{A UV expansions}

The expansions near the standard UV boundary can be computed in a straightforward manner. Let us fix the location of the boundary at $r=0$. The blackening factor $f$ is very close to unity in the UV,

$$
f(r)=1+\mathcal{O}\left(r^{4}\right)
$$

Therefore the leading finite temperature and zero temperature expansions of the various fields coincide. Moreover, as the tachyon vanishes at least linearly in $r$ for $r \rightarrow 0$, it can be set to zero when solving for the leading behavior of the coupling $\lambda$ and the warp factor $A$. Hence the expansions take the form familiar from earlier works [30-35]. We reproduce here the leading expansions of $\lambda$ and $A$ as well as the expansions of the non-normalizable and normalizable tachyon expansions both in terms of $r$ and $A$.

\section{A.1 Fields $\lambda$ and $A$}

Setting the tachyon to zero, the equations of motion for $\lambda$ and $A$ involve the effective potential

$$
V_{\mathrm{eff}}(\lambda)=V_{g}(\lambda)-x_{f} V_{f}(\lambda, 0)=\frac{12}{\mathcal{L}_{\mathrm{UV}}^{2}}\left[1+V_{1} \lambda+V_{2} \lambda^{2}+\cdots\right] .
$$


Then the (leading) UV expansions of $A$ and $\lambda$ can be found by substituting suitable Ansätze in the equations of motion. The result reads

$$
\begin{aligned}
A(r)= & -\ln \frac{r}{\mathcal{L}_{\mathrm{UV}}}+\frac{4}{9 \ln (r \Lambda)} \\
& +\frac{\frac{1}{162}\left[95-\frac{64 V_{2}}{V_{1}^{2}}\right]+\frac{1}{81} \ln [-\ln (r \Lambda)]\left[-23+\frac{64 V_{2}}{V_{1}^{2}}\right]}{\ln (r \Lambda)^{2}}+\mathcal{O}\left(\frac{1}{\ln (r \Lambda)^{3}}\right) \\
V_{1} \lambda(r)= & -\frac{8}{9 \ln (r \Lambda)}+\frac{\ln [-\ln (r \Lambda)]\left[\frac{46}{81}-\frac{128 V_{2}}{81 V_{1}^{2}}\right]}{\ln (r \Lambda)^{2}}+\mathcal{O}\left(\frac{1}{\ln (r \Lambda)^{3}}\right) .
\end{aligned}
$$

Two combinations of the series coefficients of the effective potential appear here. As the potential is matched with perturbative QCD, they become

$$
\begin{aligned}
V_{1} & =\frac{8}{9} b_{0}=\frac{88-16 x_{f}}{27} \\
\frac{V_{2}}{V_{1}^{2}} & =\frac{23}{64}+\frac{9 b_{1}}{16 b_{0}^{2}}=\frac{1}{64}\left(23+\frac{54\left(34-13 x_{f}\right)}{\left(11-2 x_{f}\right)^{2}}\right)
\end{aligned}
$$

where $b_{i}$ are the coefficients of the perturbative QCD beta function. Notice that these coefficients are indeed the same for all potentials used in our study and in particular independent of the choice of $W_{0}$.

Let us also present the expansions in terms of $A$, as we use it as a coordinate in all numerical calculations. The result after the conversion reads

$$
\begin{aligned}
\ln r(A)= & -A+\ln \left(\mathcal{L}_{\mathrm{UV}}\right)-\frac{4}{9 A} \\
& -\frac{72 \ln \left(\mathcal{L}_{\mathrm{UV}} \Lambda\right)-95+\frac{64 V_{2}}{V_{1}^{2}}+\left(46-\frac{128 V_{2}}{V_{1}^{2}}\right) \ln A}{162 A^{2}}+\mathcal{O}\left(A^{-3}\right) \\
V_{1} \lambda(A)= & \frac{8}{9 A}+\frac{\left(46-\frac{128 V_{2}}{V_{1}^{2}}\right) \ln A+72 \ln \left(\mathcal{L}_{\mathrm{UV}} \Lambda\right)}{81 A^{2}}+\mathcal{O}\left(A^{-3}\right) .
\end{aligned}
$$

\section{A.2 The tachyon}

As the tachyon is decoupled near the UV boundary, its UV behavior can be studied by inserting the expansions calculated above for $\lambda$ and $A$ into the tachyon EoM. We also develop the potentials as series in the UV:

$$
\begin{aligned}
V_{\mathrm{eff}}(\lambda) & =V_{g}(\lambda)-x_{f} V_{f}(\lambda, 0)=\frac{12}{\mathcal{L}_{\mathrm{UV}}^{2}}\left[1+V_{1} \lambda+V_{2} \lambda^{2}+\cdots\right] \\
x V_{f}(\lambda) & =W_{0}+W_{1} \lambda+W_{2} \lambda^{2}+\cdots \\
\frac{\kappa(\lambda)}{a(\lambda)} & =\frac{2 \mathcal{L}_{\mathrm{UV}}^{2}}{3}\left[1+\kappa_{1} \lambda+\kappa_{2} \lambda^{2}+\cdots\right] .
\end{aligned}
$$

Here the leading coefficient of $\kappa / a$ was already fixed in order to have the correct UV mass of the tachyon [37]. It is enough to study the linear terms in the tachyon EoM, which 
become

$$
\begin{aligned}
\tau^{\prime \prime}(r)+[ & \left.-3+\mathcal{O}\left(\frac{1}{\ln (r \Lambda)^{2}}\right)\right] \frac{\tau^{\prime}(r)}{r} \\
& +\left[3+\frac{8\left(\kappa_{1}+V_{1}\right)}{3 V_{1} \ln (r \Lambda)}+\mathcal{O}\left(\frac{1}{\ln (r \Lambda)^{2}}\right)\right] \frac{\tau(r)}{r^{2}}=0 .
\end{aligned}
$$

The general solution for $r \rightarrow 0$ reads

$$
\begin{aligned}
\frac{1}{\mathcal{L}_{\mathrm{UV}}} \tau(r)=m_{q} r & (-\ln (r \Lambda))^{\frac{4}{3}+\frac{4 \kappa_{1}}{3 V_{1}}}\left[1+\mathcal{O}\left(\frac{1}{\ln (r \Lambda)}\right)\right] \\
+ & \sigma r^{3}(-\ln (r \Lambda))^{-\frac{4}{3}-\frac{4 \kappa_{1}}{3 V_{1}}}\left[1+\mathcal{O}\left(\frac{1}{\ln (r \Lambda)}\right)\right] .
\end{aligned}
$$

Here matching with the perturbative anomalous dimension of the quark mass in QCD gives

$$
\frac{4}{3}+\frac{4 \kappa_{1}}{3 V_{1}}=-\frac{\gamma_{0}}{b_{0}}=-\frac{9}{22-4 x_{f}}
$$

where $\gamma_{0}$ is the leading coefficient of the anomalous dimension of the quark mass in QCD.

The result can be again written in terms of $A$, and it becomes

$$
\begin{aligned}
\tau(A)=m_{q} \mathcal{L}_{\mathrm{UV}}^{2} e^{-A}(\ln (A))^{-\frac{\gamma_{0}}{b_{0}}}\left[1+\mathcal{O}\left(A^{-1}\right)\right] \\
+\sigma \mathcal{L}_{\mathrm{UV}}^{4} e^{-3 A}(\ln (A))^{\frac{\gamma_{0}}{b_{0}}}\left[1+\mathcal{O}\left(A^{-1}\right)\right] .
\end{aligned}
$$

\section{A.3 Finite temperature}

The basic relations

$$
f(r)=1-\frac{\int_{0}^{r} d r / b^{3}(r)}{\int_{0}^{r_{h}} d r / b^{3}(r)}, \quad \frac{1}{4 \pi T}=b_{h}^{3} \int_{0}^{r_{h}} \frac{d r}{b^{3}(r)},
$$

can be evaluated in the UV by inserting from (A.3)

$$
b=e^{A}=\frac{\mathcal{L}_{\mathrm{UV}}}{r}\left[1+\frac{4}{9 \ln (\Lambda r)}+\frac{4}{9 \ln ^{2}(\Lambda r)}\left(\frac{b_{1}}{b_{0}^{2}} \ln (-\ln (\Lambda r))+\frac{11}{9}-\frac{b_{1}}{2 b_{0}^{2}}\right)\right] .
$$

Terms of the order of $\ln ^{2}(\ln ) / \ln ^{3}$ are neglected; for these, see [80]. One finds 20

$$
\begin{aligned}
\int_{0}^{r} \frac{d r}{b^{3}(r)} & =\frac{r^{4}}{4 \mathcal{L}_{\mathrm{UV}}^{3}}\left[1-\frac{4}{3 \ln (\Lambda r)}-\frac{4}{3 \ln ^{2}(\Lambda r)}\left(\frac{b_{1}}{b_{0}^{2}} \ln (-\ln (\Lambda r))+\frac{7}{12}-\frac{b_{1}}{2 b_{0}^{2}}\right)\right], \\
T & =\frac{1}{\pi r_{h}}\left(1+\frac{1}{3 \ln ^{2}\left(\Lambda r_{h}\right)}\right) .
\end{aligned}
$$

For the quantity $b_{h} / T$ needed for the latent heat one has

$$
\frac{b_{h}}{T}=\pi \mathcal{L}_{\mathrm{UV}}\left[1+\frac{4}{9 \ln \left(\Lambda r_{h}\right)}+\frac{4}{9 \ln ^{2}\left(\Lambda r_{h}\right)}\left(\frac{b_{1}}{b_{0}^{2}} \ln \left(-\ln \left(\Lambda r_{h}\right)\right)+\frac{17}{36}-\frac{b_{1}}{2 b_{0}^{2}}\right)\right] .
$$

\footnotetext{
${ }^{20}$ Ref. [30-32], second paper, equation (D.3), has a different constant in the expansion of $T$. We have checked the $1 / 3$ here also numerically.
} 


\section{B IR expansions}

\section{B.1 Zero temperature}

Here we first discuss the expansions near the "good" IR singularity at zero temperature. It is the particular solution which can be lifted to finite temperature. In the IR, the tachyon potential in the DBI action is expected to be exponentially suppressed. Therefore the tachyon is again decoupled, and the IR behavior of $\lambda$ and $A$ can be solved separately from that of the tachyon. Moreover, the IR expansions of $\lambda$ and $A$ are exactly the same as in IHQCD. We will anyhow repeat the discussion for the particular asymptotics of $V_{g}$ that matches well with the IR properties of QCD [30-32], which covers all potentials in this article.

\section{B.1.1 $A$ and $\lambda$}

Let us assume that the potential $V_{g}$ has the asymptotic behavior

$$
V_{g}(\lambda)=v_{0} \lambda^{4 / 3} \sqrt{\ln \lambda}\left[1+\frac{v_{1}}{\ln \lambda}+\frac{v_{2}}{\ln ^{2} \lambda}+\cdots\right] .
$$

Then the asymptotic solution reads

$$
\begin{aligned}
A= & -\frac{r^{2}}{R^{2}}+\frac{1}{2} \ln \frac{r}{R}-\ln R-\frac{1}{2} \ln v_{0}+\frac{5}{4} \ln 2+\frac{3}{4} \ln 3+\frac{23}{24}+\frac{4 v_{1}}{3} \\
& +\frac{R^{2}\left(-173+512 v_{1}^{2}+1024 v_{2}\right)}{3456 r^{2}}+\mathcal{O}\left(r^{-4}\right) \\
= & -\frac{r^{2}}{R^{2}}+\frac{1}{4} \ln \frac{3 r^{2}}{2 R^{2}}+A_{0}+\frac{23}{24}+\frac{4 v_{1}}{3} \\
& +\frac{R^{2}\left(-173+512 v_{1}^{2}+1024 v_{2}\right)}{3456 r^{2}}+\mathcal{O}\left(r^{-4}\right) \\
\ln \lambda= & \frac{3}{2} \frac{r^{2}}{R^{2}}-\frac{23}{16}-2 v_{1}-\frac{R^{2}\left(151+512 v_{1}^{2}+1024 v_{2}\right)}{2304 r^{2}}+\mathcal{O}\left(r^{-4}\right)
\end{aligned}
$$

where

$$
e^{A_{0}}=\frac{\sqrt{24}}{R \sqrt{v_{0}}} .
$$

The IR scale $R=1 / \Lambda_{\mathrm{IR}}$ is an integration constant here. ${ }^{21}$ Recall that $r$ does not appear explicitly in the equations of motion, and therefore there is also an integration constant related to $r$ : we have the freedom of shifting any solution by $r \rightarrow r+\delta r$. The solution having the simple $r$-dependence of eqs. (B.2) and (B.3) corresponds to a special choice of $\delta r$. It will have its UV boundary at an arbitrary value of $r$ (rather than at $r=0$ ). If $\delta r$ is fixed instead by requiring the UV boundary to lie at $r=0$, a corresponding shift must be added to the asymptotic formulas. For our choice of $V_{g}$,

$$
\begin{aligned}
& v_{0}=\frac{92\left(b_{0}^{\mathrm{YM}}\right)^{2}-144 b_{1}^{\mathrm{YM}}}{27 \mathcal{L}_{0}^{2}}=\frac{18476}{243} \\
& v_{1}=\frac{1}{2} ; \quad v_{2}=-\frac{1}{8}
\end{aligned}
$$

if we set $\mathcal{L}_{0}=1$.

\footnotetext{
${ }^{21}$ It is not independent of the scale $\Lambda=\Lambda_{\mathrm{UV}}$ of the UV expansions, i.e., the complete solution from UV to the IR will have fixed $\Lambda_{\mathrm{UV}} / \Lambda_{\mathrm{IR}}$.
} 
Using $A$ as the coordinate, the result reads

$$
\begin{aligned}
\frac{r^{2}}{R^{2}}= & -A+\frac{1}{4} \ln \left(-\frac{3}{2} A\right)+A_{0}+\frac{23}{24}+\frac{4 v_{1}}{3} \\
& -\frac{655+1152 v_{1}+512 v_{1}^{2}+1024 v_{2}}{3456 A}-\frac{\ln \left(-\frac{3}{2} A\right)+4 A_{0}}{16 A}+\mathcal{O}\left(A^{-2}\right) \\
\ln \lambda= & -\frac{3}{2} A+\frac{3}{8} \ln \left(-\frac{3}{2} A\right)+\frac{3}{2} A_{0}-\frac{7+16 v_{1}+3 \ln \left(-\frac{3}{2} A\right)+12 A_{0}}{32 A}+\mathcal{O}\left(A^{-2}\right)
\end{aligned}
$$

Various other combinations may be useful. In thermodynamics one needs $b=e^{A}$ in terms of $\lambda$; from (B.9) one can invert:

$$
b=\left(\frac{3}{2}\right)^{3 / 4} \frac{4}{R \sqrt{v_{0}}} \frac{1}{\lambda^{2 / 3}}\left(\frac{2}{3} \ln \lambda\right)^{1 / 4}\left[1+\mathcal{O}\left(\frac{1}{\ln \lambda}\right)\right]
$$

For the pair of functions $q(A)=e^{A} r^{\prime}(A), \lambda(A)$ used in numerics one can derive, for $A \rightarrow-\infty$,

$$
\begin{aligned}
& q(A)=-\frac{R}{2} e^{A}(-A)^{-1 / 2}\left[1+\frac{1}{8 A}\left(\ln \left(-\frac{3}{2} A\right)+4 A_{0}+\frac{9}{2}\right)+\mathcal{O}\left(A^{-2}\right)\right] \\
& \lambda(A)=e^{-\frac{3}{2}\left(A-A_{0}\right)}\left(-\frac{3}{2} A\right)^{3 / 8}\left[1-\frac{3}{32 A}\left(\ln \left(-\frac{3}{2} A\right)+4 A_{0}+5\right)+\mathcal{O}\left(A^{-2}\right)\right] .
\end{aligned}
$$

\section{B.1.2 The tachyon}

The IR expansion of the tachyon depends on the large- $\lambda$ asymptotics of the potentials $V_{f}$, $a$, and $\kappa$. Recall that the tachyon potential $V_{f}(\lambda, \tau)$ needs to vanish in the IR [37] in order to have correct kind of flavor anomalies. All power-law asymptotics for the potentials were analyzed in [26], and two different acceptable cases were chosen as examples. They are:

I Asymptotics with

$$
a(\lambda) \sim \lambda^{0} ; \quad \kappa(\lambda) \sim \lambda^{4 / 3} ; \quad V_{f 0}(\lambda) \sim \lambda^{\hat{\tau}}
$$

where $\hat{\tau}<10 / 3$. This case includes the potentials I and $\mathrm{I}_{*}$ of this article (for which $\hat{\tau}=2)$. The tachyon diverges exponentially for $r \rightarrow \infty(A \rightarrow-\infty)$,

$$
\tau \sim e^{C_{I} \frac{r}{R}} \sim e^{C_{I} \sqrt{-A}}
$$

where the coefficient reads for potentials I

$$
C_{I}=\frac{813^{5 / 6}\left(115-16 x_{f}\right)^{4 / 3}\left(11-x_{f}\right)}{8129442^{1 / 6}} .
$$

II Asymptotics with

$$
a(\lambda) \sim \lambda^{\hat{\sigma}} ; \quad \kappa(\lambda) \sim \lambda^{4 / 3} ; \quad V_{f 0}(\lambda) \sim \lambda^{\hat{\tau}}
$$


where $\hat{\sigma}>0$ and $\hat{\tau}$ can take any value. This case includes the potentials II and $\mathrm{II}_{*}$ of this article (for which $\hat{\sigma}=2 / 3$ and $\hat{\tau}=2$ ). The tachyon diverges for $r \rightarrow \infty$ $(A \rightarrow-\infty)$ as

$$
\tau \sim C_{I I} \sqrt{\frac{r}{R}} \sim C_{I I}(-A)^{1 / 4}
$$

where the coefficient reads for potentials II

$$
C_{I I}=\frac{272^{3 / 4} 3^{1 / 4}}{\sqrt{4619}}
$$

\section{B.2 Finite temperature}

We will work out the finite temperature IR expansions in $A$-coordinates. Instead of writing down the explicit expansions as above, it is more convenient to state the relations between the coefficients of the series expansions. We start by defining the series

$$
\begin{aligned}
f & =\epsilon f_{h}^{\prime}+\mathcal{O}\left(\epsilon^{2}\right), \quad f^{\prime}(0)=f_{h}^{\prime}+\epsilon f_{h}^{\prime \prime}+\mathcal{O}\left(\epsilon^{2}\right), \\
q & =q_{h}+\epsilon q_{h}^{\prime}+\mathcal{O}\left(\epsilon^{2}\right), \\
\lambda & =\lambda_{h}+\epsilon \lambda_{h}^{\prime}+\mathcal{O}\left(\epsilon^{2}\right), \\
\tau & =\tau_{h}+\epsilon \tau_{h}^{\prime}+\frac{1}{2} \epsilon^{2} \tau_{h}^{\prime \prime}+\mathcal{O}\left(\epsilon^{3}\right),
\end{aligned}
$$

where $\epsilon=A-A_{h}$ is the distance from the horizon, which lies at $A=A_{h}$, and all coefficients are to be evaluated at the horizon. The key input here is $f\left(A_{h}\right)=0$. Inserting to the equations of motion one can solve for six of the nine coefficients listed above:

$$
\begin{aligned}
q_{h} & =-\frac{\sqrt{3 f_{h}^{\prime}}}{\sqrt{V_{g}-V_{f}}} \\
f_{h}^{\prime \prime} & =-4 f_{h}^{\prime}+\frac{q_{h}^{4}}{f_{h}^{\prime}}\left[\frac{1}{16} \lambda_{h}^{2}\left(\partial_{\lambda} V_{g}-\partial_{\lambda} V_{f}\right)^{2}+\frac{\left(\partial_{\tau} V_{f}\right)^{2}}{6 V_{f} \kappa_{h}}\right] \\
q_{h}^{\prime} & =\frac{q_{h}^{5}}{\left(f_{h}^{\prime}\right)^{2}}\left[\frac{1}{16} \lambda_{h}^{2}\left(\partial_{\lambda} V_{g}-\partial_{\lambda} V_{f}\right)^{2}+\frac{\left(\partial_{\tau} V_{f}\right)^{2}}{6 V_{f} \kappa_{h}}\right]=q_{h}\left(4+\frac{f_{h}^{\prime \prime}}{f_{h}^{\prime}}\right) \\
\lambda_{h}^{\prime} & =-\frac{3 \lambda_{h}^{2} q_{h}^{2}}{8 f_{h}^{\prime}}\left(\partial_{\lambda} V_{g}-\partial_{\lambda} V_{f}\right) \\
\tau_{h}^{\prime} & =\frac{q_{h}^{2} \partial_{\tau} \ln V_{f}}{f_{h}^{\prime} \kappa_{h}} \\
\tau_{h}^{\prime \prime} & =\frac{9 \partial_{\tau} V_{f}(A+B+C)+D}{12 \kappa_{h}^{2} V_{f}^{3}\left(V_{f}-V_{g}\right)^{3}}
\end{aligned}
$$

with the abbreviations

$$
\begin{aligned}
A & =6 \lambda_{h}^{2} \kappa_{h}^{\prime} V_{f}^{3}\left(\partial_{\lambda} V_{g}-\partial_{\lambda} V_{f}\right), \\
B & =V_{f}^{2}\left[8 \partial_{\tau}^{2} V_{f}-3 \lambda_{h}^{2}\left(\partial_{\lambda} V_{g}-\partial_{\lambda} V_{f}\right)\left(\kappa_{h}\left(\partial_{\lambda} V_{g}-3 \partial_{\lambda} V_{f}\right)+2 \kappa_{h}^{\prime} V_{g}\right)\right], \\
C & =-2 V_{f}\left[6 \partial_{\tau} V_{f}+V_{g}\left(4 \partial_{\tau}^{2} V_{f}+3 \lambda_{h}^{2} \kappa_{h} \partial_{\lambda} V_{f}\left(\partial_{\lambda} V_{g}-\partial_{\lambda} V_{f}\right)\right)\right], \\
D & =27 \lambda_{h}^{2} \kappa_{h} \partial_{\tau} \partial_{\lambda} V_{f} V_{f}^{2}\left(V_{g}-V_{f}\right)\left(\partial_{\lambda} V_{g}-\partial_{\lambda} V_{f}\right) .
\end{aligned}
$$


Here $V_{g} \equiv V_{g}\left(\lambda_{h}\right), V_{f} \equiv V_{f}\left(\lambda_{h}, \tau_{h}\right), \kappa_{h}^{\prime}=d \kappa\left(\lambda_{h}\right) / d \lambda, \kappa_{h}=\kappa\left(\lambda_{h}\right)$. The so far unspecified three coefficients $\lambda_{h}, \tau_{h}$, and $f_{h}^{\prime}$ remain as free parameters. However $f_{h}^{\prime}$ will be fixed by requiring the standard normalization of the blackening factor $f \rightarrow 1$ in the UV. Therefore the physically relevant parameters are $\lambda_{h}$ and $\tau_{h}$, which can be mapped to the temperature and the quark mass after the full solution has been found.

\section{The quark mass and the Efimov solutions}

As detailed in [26], the existence of the Efimov vacua is tightly linked to the tachyon mass at the IR fixed point, plotted in figure 3. In particular, the existence of the full Efimov tower of vacua with arbitrary number of tachyon nodes is guaranteed if the tachyon mass violates the BF bound. The same holds at finite temperature: one can always tune $\lambda_{h}$ and $\tau_{h}$ such that the solution comes arbitrarily close to the fixed point. When the BF bound is violated, the tachyon solution is oscillatory in the vicinity of the fixed point. Thus, when approaching the fixed point the tachyon will achieve arbitrary many nodes, which signals the presence of the full Efimov tower. In this case the dependence of the quark mass on $\lambda_{h}$ and $\tau_{h}$ is the "standard" one, i.e., qualitatively as in figure 5.

There are, however, some cases where either the fixed point is absent, which is the case for potentials $\mathrm{I}_{*}$ and $\mathrm{II}_{*}$ at low $x_{f}$, or the BF bound is not violated, which is the case, interestingly, for potentials I at very low $x_{f}$ (as well as in the conformal window for all potentials). In such cases the picture can be different from figure 5 . We shall not give a detailed description of all possible cases here, but rather discuss some of the main features and give examples.

The curve $\tau_{h c}$ (which actually starts at $\lambda_{*}$ ) exists if and only if there is a fixed point. If there is no fixed point, the solutions are expected to reach the standard UV boundary for all values of $\lambda_{h}$ and $\tau_{h}$. For the curves $\tau_{h 0}, \tau_{h 1}, \ldots$ the situation is more complicated. At least few of these curves may still exist even if there is no fixed point or if the BF bound is satisfied at the fixed point. Their existence at asymptotically large $\lambda_{h}$ is linked to the existence of Efimov solutions at zero temperature: taking $\lambda_{h} \rightarrow \infty$ with $\tau_{h}$ fixed along the curves, the finite temperature Efimov configurations converge towards their zero temperature counterparts. In particular, we expect that the chiral symmetry is broken at zero temperature if and only if $\tau_{h 0}$ exists at asymptotically large $\lambda_{h}$. We have found numerically that the curves are always absent in the conformal window, $x_{f} \geq x_{c}$, so that chiral symmetry is intact. This turns out to be the case also for potentials I at large $W_{0}$ and low $x_{f}$, but only in a part of the region where BF bound is satisfied at the fixed point. See also the phase diagrams in figure 18 of section 4 which show that chiral symmetry is intact at low $x_{f}$. For potentials $\mathrm{I}_{*}$ and at low $x_{f}$, where no fixed point exists, the curves are also absent, and chiral symmetry is unbroken. In this case the $m_{q}=0$ thermodynamics is determined by the $\tau=0$ solution and is qualitatively similar to the Yang-Mills one (see also figure 19 ). For potentials $\mathrm{II}_{*}$ however, at least the leading solution $\tau_{h 0}$ can always be found and chiral symmetry is thus broken at low temperatures (see figure 17). 


\section{Computation of $\lambda_{\text {end }}$}

One can also illustrate the connection of the behavior of $\lambda_{\text {end }}$ to the BF bound of the tachyon (figure 3), assuming that we have chosen a set of potentials and value of $x_{f}$ such that the IR fixed point exists. First we recall that $\lambda_{\text {end }}$ can be defined as the endpoint of the $\tau_{h 0}\left(\lambda_{h}\right)$ curve which gives the (non-node) solution with nontrivial tachyon and zero quark mass (figure 5). In particular, as $\lambda_{h}$ approaches $\lambda_{\text {end }}$ from above, $\tau_{h}$ tends to zero, and we expect that the whole tachyon solution from the boundary to the horizon becomes small, and the tachyon decouples from the other fields. Therefore, in order to define $\lambda_{\text {end }}$ it is enough to study the behavior of the tachyon based on the linearized tachyon EoM, evaluated on a fixed background, obtained by setting the tachyon to zero.

The linearized tachyon equation has the form

$$
\tau^{\prime \prime}(r)+F_{1} \tau^{\prime}(r)+F_{2} \tau(r)=0
$$

where

$$
\begin{aligned}
& F_{1}=3 A^{\prime}(r)+\frac{f^{\prime}(r)}{f(r)}+\lambda^{\prime}(r) \frac{\partial \ln \kappa(\lambda)}{\partial \lambda}+\lambda^{\prime}(r) \frac{\partial \ln V_{f 0}(\lambda)}{\partial \lambda} \\
& F_{2}=\frac{2 e^{2 A} a(\lambda)}{f(r) \kappa(\lambda)} .
\end{aligned}
$$

Here $A(r), \lambda(r)$, and $f(r)$ are the solutions of the EoMs for $\tau \equiv 0$, which are the same for potentials I and II. The drastic difference between the potentials, as suggested by figure 3 , thus arises only through the appearances of $a$ and $\kappa$ in the coefficients (D.2). The regular tachyon solution, which is finite in the IR, obeys

$$
\frac{\tau^{\prime}\left(r_{h}\right)}{\tau\left(r_{h}\right)}=-\lim _{r \rightarrow r_{h}} \frac{F_{2}}{F_{1}}
$$

since the double-derivative term in (D.1) is negligible near the horizon.

Nodes of the regular solution to the linear tachyon equation can then be used to determine $\lambda_{\text {end }}$. For small $\lambda_{h}$ perturbative analysis applies and it is not difficult to see that the solution is monotonic, without nodes. When $\lambda_{h}$ increases the equation becomes nontrivial and has to be studied numerically. Usually we observe, that beyond a critical value of $\lambda_{h}$ a tachyon node appears in the UV. The leading tachyon behavior in the UV is controlled by the quark mass, which has to vanish at the critical value. We thus identify the critical value as $\lambda_{\text {end }}$, which was defined as the endpoint of the curve where $m_{q}=0$. Thus the regular solution to the linearized EoM has no nodes for $\lambda_{h}<\lambda_{\text {end }}$ and one or more nodes for $\lambda_{h}>\lambda_{\text {end }}$. It can also happen that $\lambda_{\text {end }}$ does not exist, and the tachyon nodes are absent for all $\lambda_{h}$.

Since $\lambda_{h}$ can take values from zero to $\lambda_{*}$, we can construct backgrounds which get arbitrarily close to the IR fixed point at $\lambda=\lambda_{*}$. If the BF bound for the tachyon is violated at the fixed point, the tachyon must have nodes as $\lambda_{h} \rightarrow \lambda_{*}$. We can conclude that $\lambda_{\text {end }}$, and thus also the curve $\tau_{h 0}$, exist in this case. This makes sense, since when the BF bound is violated, chiral symmetry breaking takes place also at zero temperature, which means the the curve $\tau_{h 0}$ exist also at asymptotically large $\lambda_{h}$ as discussed in appendix C. 
We can also say something about $\lambda_{\text {end }}$ in the probe limit $x_{f} \rightarrow 0$. For PotII it seems that it approaches a fixed value as seen from figure 22 (right). This value can be found by solving the linearized tachyon EoM with a background evaluated at $x_{f}=0$ (i.e., the IHQCD solution), and by checking if a special value of $\lambda_{h}$ (identified as $\lambda_{\text {end }}$ ) can be found where nodes emerge in the tachyon solution. Notice that $\lambda_{*}$ goes to $\infty$ in the probe limit so that $\lambda_{h}$ can take any value. Existence of the limiting value of $\lambda_{\text {end }}$ as $x_{f} \rightarrow 0$ thus requires that the tachyon has nodes in the limit $\lambda_{h} \rightarrow \infty$ after first taking the probe limit. Since zero temperature solutions are obtained for $\lambda_{h} \rightarrow \infty$, it is plausible that the behavior of figure 22 (right) is seen if and only if the probe limit system admits tachyon solutions with nodes (in other words, chiral symmetry breaking) at zero temperature. Recall that for PotI, for which the different behavior of figure 22 (left) is found, chiral symmetry is unbroken at low $x_{f}$.

\section{E Computation of the condensate}

In principle, the condensate for an $m_{q}=0$ system could be computed from the UV expansion

$$
\tau(r) / \mathcal{L}_{\mathrm{UV}}=\sigma r^{3}(-\ln (\Lambda r))^{3 /\left(2 b_{0}\right)}, \quad r \rightarrow 0
$$

with

$$
A-\ln \left(\Lambda \mathcal{L}_{\mathrm{UV}}\right)=\frac{1}{b_{0} \lambda(A)}+\frac{b_{1}}{b_{0}^{2}} \ln \left(b_{0} \lambda(A)\right)=-\ln (\Lambda r), \quad A \rightarrow \infty,
$$

where we dropped corrections of $\mathcal{O}\left(A^{-1}\right)$. Using this one can define

$$
\ln \tilde{\sigma}(A)=\ln \tau(A)-\ln \mathcal{L}_{\mathrm{UV}}+\frac{3}{b_{0} \lambda(A)}+\frac{3 b_{1}}{b_{0}^{2}} \ln \left(b_{0} \lambda(A)\right)+\frac{3}{2 b_{0}} \ln \left(b_{0} \lambda(A)\right),
$$

which approaches $\ln \sigma$ for $A \rightarrow \infty$.

However, our solution for the tachyon, which is obtained numerically by shooting from the IR, will have a linear term $\tau \sim m_{q} r$ with a tiny quark mass (typically $m_{q} \sim 10^{-7}$ ), because the IR boundary conditions cannot be fine tuned beyond the numerical accuracy of the code. The linear term will dominate over the cubic one of eq. (E.2) in the deep UV. In order to calculate the condensate, we need to separate the linear and cubic terms from the numerically computed $\tau(A)$, and use the cubic solution in eq. (E.3). For $\tilde{\sigma}(A)$ to be a good approximation to the condensate $\sigma$, we need to have $A \sim$ hundreds. Direct separation of the linear $m_{q}$ term in this region requires numerical accuracy on the level of $e^{- \text {hundreds }}$, which is practically impossible to achieve.

To illustrate the difficulty and its resolution, consider a concrete case. Let us take Potential II, SB normalised, $\mathcal{L}_{\mathrm{UV}}=\left(1+\frac{7}{4} x_{f}\right)^{1 / 3}, x_{f}=3$. This system, when cooled, has a 2 nd order transition at $T_{\text {end }}=1.158 T_{h}$, above a 1 st order transition at $T_{h}$. This is concretely seen in figure 9 . Since chiral symmetry is broken at $T_{\text {end }}$ we expect that $\sigma(T)$ starts growing from zero at $T_{\text {end }}$ and grows when the system is cooled towards $T_{h}$. As an example, we evaluate the condensate when $T$ has been cooled to $T=0.95 T_{\text {end }}=1.1 T_{h}$.

Numerical solution of Einstein's equations required knowing the values of $\lambda_{h}, \tau_{h}$ leading to a certain $T$ with $m_{q}=0$. For this potential and $T$ they were $\lambda_{h}=0.4017564, \tau_{h}=$ 
$\tau_{h 0}\left(\lambda_{h}, m_{q}=0\right)=0.217984$. The computed $\tau(A)$ is shown in figure 28. For $A$ up to about 10 one discerns the required $r^{3} \sim e^{-3 A}$ behavior, but beyond that $r \sim e^{-A}$ sets in and extends up to the end point of the computation at $A=400$. It is impossible to shoot from the horizon and get $m_{q}=0$ more accurately; note that the tachyon has already decreased to $10^{-14}$ from 0.22 at the horizon.

To impose $\tau(r) \sim r^{3}$ one must shoot from the boundary, $r=0, A=\infty$. In this limit the evolution of $\tau$ decouples from the other bulk fields, of which only $\lambda(A)$ is relevant since $f \approx 1$. We can thus integrate the tachyon equation from some large $A(=400)$ using the $\lambda(A)$ from Einstein's equations and imposing as the initial condition $\tau(A)=e^{-3 A}, \tau^{\prime}(A)=$ $-3 e^{-3 A}$ with small enough normalisation. ${ }^{22}$ The result is plotted as the curve $\tau_{\mathrm{UV}}(A)$ in figure 28. One observes that in the range $A=2 \ldots 10$ the curve behaves accurately as a constant $\times \tau_{\mathrm{IR}}(A)$ and the normalisation can thus be determined. In this way the true $\tau\left(A ; m_{q}=0\right)$ plotted in figure 28 is obtained.

Now that the accurate $\tau(A)$ is known, $\ln \tilde{\sigma}(A)$ can be plotted using eq. (E.3), see figure 28. For the extrapolation it is even more convenient to plot as a function of $\lambda(A)$, see also figure 28. One obtains a nice linear behavior with the asymptotic value $\ln \sigma=$ $-5.1558, \sigma\left(T=0.95 T_{\text {end }}\right)=0.005766$.

If one used the original $\tau\left(A, m_{q}=0\right)$ at the largest value of $A, A=10$, where the $r^{3}$ behavior was obtained, one would have $\tilde{\sigma}=0.0106$. This is too large by a factor 1.82 , not very far off, but actually slightly larger than the expected $10 \%$ error from neglecting the $\mathcal{O}\left(A^{-1}\right)$ corrections in eqs. (E.2) and (E.3) at this value of $A$. If we tried using the $\tau\left(A, m_{q}=0\right)$ solution directly, reliable extraction of $\sigma$ would thus require much higher numerical precision, as already mentioned above.

After applying the procedure discussed above, the dominant error in the value of $\sigma$ arises actually from the matching of the two tachyon solutions that were obtained by shooting from the UV and from the IR. The solutions are not exactly proportional for $0 \lesssim A \lesssim 10$ due to nonlinearities in the tachyon EoM and coupling to other fields. The error can be reduced by introducing a further subtraction trick that effectively reduces the value of $m_{q}$ of the solution that was obtained by shooting form the IR, so that the matching can be done for slightly higher values of $A$ where the coupling effects are considerably reduced.

We follow [26] and construct two solutions $\tau_{1,2}$ with small but different values $m_{q 1, q 2}$. Optimal choice is to take $\left|m_{q}\right|$ as small as possible and choose one solution with a positive value and another with a negative one. Then we construct

$$
\tau_{\mathrm{IR}}(A)=\frac{1}{1-\frac{m_{q 1}}{m_{q 2}}}\left(\tau_{1}(A)-\frac{m_{q 1}}{m_{q 2}} \tau_{2}(A)\right)
$$

where the ratio $m_{q 1} / m_{q 2}$ can be accurately determined as the ratio of the solutions $\tau_{1} / \tau_{2}$ at

\footnotetext{
${ }^{22}$ It is not important to have precisely correct UV boundary conditions, since corrections to the $\tau \sim r^{3}$ solution will decay fast as the system is solved toward the IR. One should only make sure that the tachyon is much less than one in the whole region of interest $(A \gtrsim 0)$ in order to suppress nonlinear effects, or alternatively use explicitly linearized differential equation for the tachyon.
} 


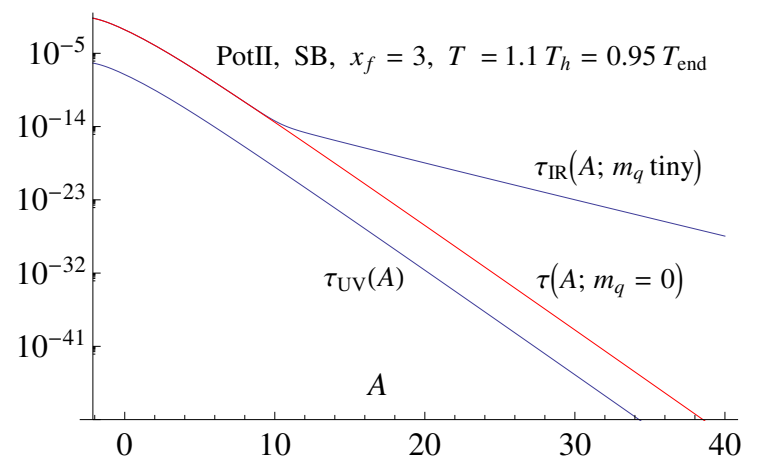

Figure 28. The tachyon computed for $T=0.95 T_{\text {end }}=1.1 T_{h}$. The curve $\tau_{\mathrm{IR}}\left(A, m_{q}=\right.$ tiny) is obtained by integrating Einstein's equations from the horizon and tuning $m_{q}=0$ as accurately as possible. The curve $\tau_{\mathrm{UV}}(A)$ is obtained by integrating the tachyon equation of motion from the $\mathrm{UV}$ at $A=400$ using the bulk field $\lambda(A)$ from Einstein's equation and imposing $\tau \sim r^{3}$ in the UV. The normalisation can be fixed by matching to $\tau_{\mathrm{IR}}$ in the $A=2 \ldots 10$ range and a reliable $\tau\left(A, m_{q}=0\right)$ for the true $m_{q}=0$ tachyon is obtained.
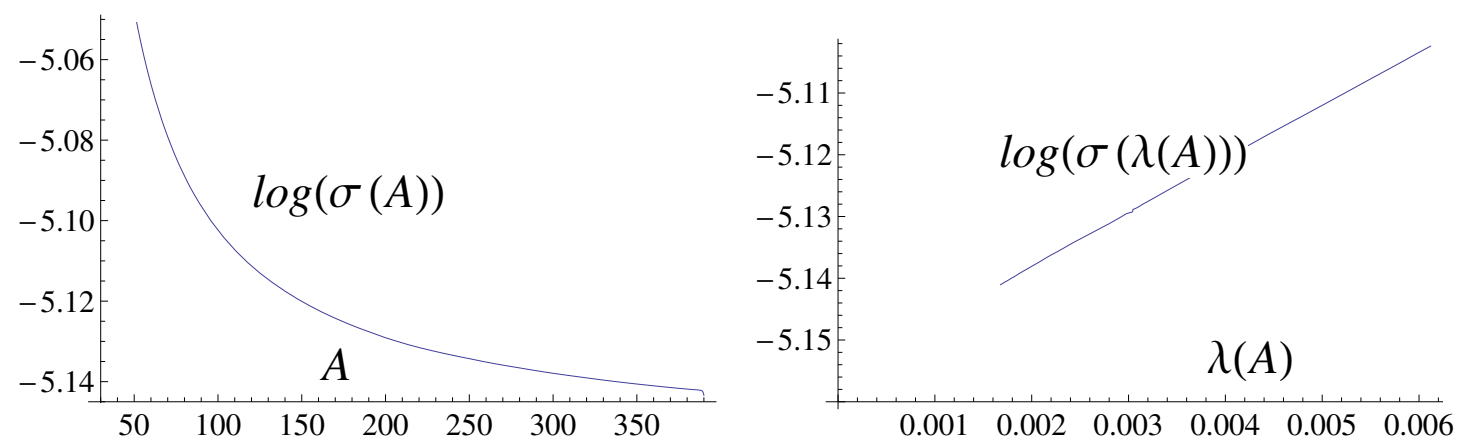

Figure 29. Extrapolation of the value of $\ln \sigma$ to $r=0, A=\infty, \lambda=0$, for the potential and temperature as in figure 28

large $A$ (say $A=400) .{ }^{23}$ The point is that the constructed $\tau_{\mathrm{IR}}$ has its $m_{q}$ several orders of magnitude closer to zero than either of the solutions $\tau_{1,2}$. Moreover, the residual dependence of $\sigma$ on $m_{q}$ is drastically reduced: the linear corrections cancel in (E.4) (see [26]). The improved value of $\sigma$ can now be found by matching $\tau_{\mathrm{IR}}$ with the solution $\tau_{\mathrm{UV}}$, which was obtained by shooting from the UV, as discussed above.

Open Access. This article is distributed under the terms of the Creative Commons Attribution License which permits any use, distribution and reproduction in any medium, provided the original author(s) and source are credited.

\footnotetext{
${ }^{23}$ There is a small technicality involved in this procedure as the two solutions will in general have different values of $\Lambda$. Changing $\Lambda$ is equivalent with shifts of $A$ in $A$-coordinates (see eq. (E.2)), so we can fix the issue by shifting, say, the solution $\tau_{2}$ by a small $\Delta A$, obtained by requiring that the corresponding solutions $\lambda_{1,2}$ for the coupling match at large $A$. Notice also that the resulting $\tau_{\mathrm{IR}}$ is only useful in the UV region $A \gtrsim 0$.
} 


\section{References}

[1] G. Veneziano, U(1) without instantons, Nucl. Phys. B 159 (1979) 213 [inSPIRE].

[2] T. Banks and A. Zaks, On the phase structure of vector-like gauge theories with massless fermions, Nucl. Phys. B 196 (1982) 189 [inSPIRE].

[3] W.E. Caswell, Asymptotic behavior of nonabelian gauge theories to two loop order, Phys. Rev. Lett. 33 (1974) 244 [INSPIRE].

[4] D.R.T. Jones, Two loop diagrams in Yang-Mills theory, Nucl. Phys. B 75 (1974) 531 [INSPIRE].

[5] V.L. Berezinskii, Destruction of long-range order in one-dimensional and two-dimensional systems having a continuous symmetry group. I. Classical systems, Zh. Eksp. Teor. Fiz. 59 (1970) 907 [Sov. Phys. JETP 32 (1971) 493].

[6] J. Kosterlitz and D. Thouless, Ordering, metastability and phase transitions in two-dimensional systems, J. Phys. C 6 (1973) 1181 [INSPIRE].

[7] V.A. Miransky, Dynamics of spontaneous chiral symmetry breaking and continuum limit in quantum electrodynamics, Nuovo Cim. A90 (1985) 149.

[8] M.G. Alford, K. Rajagopal and F. Wilczek, QCD at finite baryon density: nucleon droplets and color superconductivity, Phys. Lett. B 422 (1998) 247 [hep-ph/9711395] [INSPIRE].

[9] M.G. Alford, K. Rajagopal and F. Wilczek, Color flavor locking and chiral symmetry breaking in high density QCD, Nucl. Phys. B 537 (1999) 443 [hep-ph/9804403] [InSPIRE].

[10] J. Giedt, Lattice gauge theory and physics beyond the standard model, talk given at the $30^{\text {th }}$ International Symposium on Lattice Field Theory (Lattice2012), June 24-29, Cairns, Australia (2012).

[11] M. Panero, Recent results in large- $N$ lattice gauge theories, talk given at the $30^{\text {th }}$ International Symposium on Lattice Field Theory (Lattice2012), June 24-29, Cairns, Australia (2012).

[12] M. Teper, Large- $N$ and confining flux tubes as strings $-A$ view from the lattice, Acta Phys. Polon. B 40 (2009) 3249 [arXiv: 0912.3339] [INSPIRE].

[13] B. Lucini and M. Panero, SU(N) gauge theories at large- $N$, arXiv:1210.4997 [InSPIRE].

[14] Z. Fodor et al., Twelve massless flavors and three colors below the conformal window, Phys. Lett. B 703 (2011) 348 [arXiv:1104.3124] [INSPIRE].

[15] X.-Y. Jin and R.D. Mawhinney, Evidence for a first order, finite temperature phase transition in 8 flavor QCD, PoS(LATTICE 2010) 055 [arXiv: 1011.1511] [INSPIRE].

[16] K. Miura, M.P. Lombardo and E. Pallante, Chiral phase transition at finite temperature and conformal dynamics in large $N_{f}$ QCD, Phys. Lett. B 710 (2012) 676 [arXiv:1110.3152] [INSPIRE].

[17] J. Kogut and D. Sinclair, Thermodynamics of lattice QCD with 3 flavours of colour-sextet quarks, Phys. Rev. D 85 (2012) 054505 [arXiv:1111.3353] [INSPIRE].

[18] R.D. Pisarski, Finite temperature QCD at large-N, Phys. Rev. D 29 (1984) 1222 [INSPIRE].

[19] A. Dumitru and R.D. Pisarski, Degrees of freedom and the deconfining phase transition, Phys. Lett. B 525 (2002) 95 [hep-ph/0106176] [INSPIRE]. 
[20] T. Appelquist, A. Ratnaweera, J. Terning and L. Wijewardhana, The phase structure of an $\mathrm{SU}(N)$ gauge theory with $N_{f}$ flavors, Phys. Rev. D 58 (1998) 105017 [hep-ph/9806472] [INSPIRE].

[21] F. Sannino and J. Schechter, Chiral phase transition for $\mathrm{SU}(N)$ gauge theories via an effective Lagrangian approach, Phys. Rev. D 60 (1999) 056004 [hep-ph/9903359] [INSPIRE].

[22] J.T. Lenaghan, D.H. Rischke and J. Schaffner-Bielich, Chiral symmetry restoration at nonzero temperature in the $\mathrm{SU}(3)(r) \times \mathrm{SU}(3)(l)$ linear $\sigma$-model, Phys. Rev. D 62 (2000) 085008 [nucl-th/0004006] [INSPIRE].

[23] P. Calabrese and P. Parruccini, Five loop $\epsilon$-expansion for $\mathrm{U}(n) \times \mathrm{U}(m)$ models: finite temperature phase transition in light QCD, JHEP 05 (2004) 018 [hep-ph/0403140] [INSPIRE].

[24] A. Karch and A. O'Bannon, Chiral transition of $N=4$ super Yang-Mills with flavor on a 3-sphere, Phys. Rev. D 74 (2006) 085033 [hep-th/0605120] [INSPIRE].

[25] Y. Kikukawa, M. Kohda and J. Yasuda, First-order restoration of $\mathrm{SU}(N F) \times \mathrm{SU}\left(N_{f}\right)$ chiral symmetry with large- $N_{f}$ and electroweak phase transition, Phys. Rev. D 77 (2008) 015014 [arXiv:0709.2221] [INSPIRE].

[26] M. Jarvinen and E. Kiritsis, Holographic models for QCD in the Veneziano limit, JHEP 03 (2012) 002 [arXiv:1112.1261] [INSPIRE].

[27] R. Narayanan and H. Neuberger, Chiral symmetry restoration at finite temperature in the planar limit, Phys. Lett. B 638 (2006) 546 [hep-th/0605173] [INSPIRE].

[28] L. McLerran and R.D. Pisarski, Phases of cold, dense quarks at large- $N_{c}$, Nucl. Phys. A 796 (2007) 83 [arXiv: 0706 .2191] [INSPIRE].

[29] T. Kahara and K. Tuominen, Effective models of two-flavor QCD: finite $\mu$ and $m_{q}$-dependence, Phys. Rev. D 82 (2010) 114026 [arXiv:1006.3931] [INSPIRE].

[30] U. Gürsoy and E. Kiritsis, Exploring improved holographic theories for QCD: part I, JHEP 02 (2008) 032 [arXiv:0707.1324] [INSPIRE].

[31] U. Gürsoy, E. Kiritsis and F. Nitti, Exploring improved holographic theories for QCD: part II, JHEP 02 (2008) 019 [arXiv:0707.1349] [INSPIRE].

[32] E. Kiritsis, Dissecting the string theory dual of QCD, Fortsch. Phys. 57 (2009) 396 [arXiv: 0901.1772] [INSPIRE].

[33] U. Gürsoy, E. Kiritsis, L. Mazzanti and F. Nitti, Deconfinement and gluon plasma dynamics in improved holographic QCD, Phys. Rev. Lett. 101 (2008) 181601 [arXiv:0804.0899] [INSPIRE].

[34] U. Gürsoy, E. Kiritsis, L. Mazzanti and F. Nitti, Holography and thermodynamics of $5 D$ dilaton-gravity, JHEP 05 (2009) 033 [arXiv:0812.0792] [INSPIRE].

[35] U. Gürsoy, E. Kiritsis, L. Mazzanti and F. Nitti, Improved holographic Yang-Mills at finite temperature: comparison with data, Nucl. Phys. B 820 (2009) 148 [arXiv:0903.2859] [INSPIRE].

[36] U. Gürsoy, E. Kiritsis, L. Mazzanti, G. Michalogiorgakis and F. Nitti, Improved holographic QCD, Lect. Notes Phys. 828 (2011) 79 [arXiv: 1006.5461] [INSPIRE].

[37] R. Casero, E. Kiritsis and A. Paredes, Chiral symmetry breaking as open string tachyon condensation, Nucl. Phys. B 787 (2007) 98 [hep-th/0702155] [INSPIRE]. 
[38] I. Iatrakis, E. Kiritsis and A. Paredes, An AdS/QCD model from Sen's tachyon action, Phys. Rev. D 81 (2010) 115004 [arXiv: 1003.2377] [INSPIRE].

[39] I. Iatrakis, E. Kiritsis and A. Paredes, An AdS/QCD model from tachyon condensation: II, JHEP 11 (2010) 123 [arXiv:1010.1364] [INSPIRE].

[40] F. Bigazzi, R. Casero, A. Cotrone, E. Kiritsis and A. Paredes, Non-critical holography and four-dimensional CFT's with fundamentals, JHEP 10 (2005) 012 [hep-th/0505140] [INSPIRE].

[41] J. Alanen, K. Kajantie and V. Suur-Uski, Spatial string tension of finite temperature QCD matter in gauge/gravity duality, Phys. Rev. D 80 (2009) 075017 [arXiv:0905.2032] [INSPIRE].

[42] J. Alanen, K. Kajantie and V. Suur-Uski, A gauge/gravity duality model for gauge theory thermodynamics, Phys. Rev. D 80 (2009) 126008 [arXiv:0911.2114] [INSPIRE].

[43] S.S. Gubser and A. Nellore, Mimicking the QCD equation of state with a dual black hole, Phys. Rev. D 78 (2008) 086007 [arXiv:0804.0434] [INSPIRE].

[44] O. DeWolfe, S.S. Gubser and C. Rosen, A holographic critical point, Phys. Rev. D 83 (2011) 086005 [arXiv: 1012.1864 ] [inSPIRE].

[45] J. Alanen and K. Kajantie, Thermodynamics of a field theory with infrared fixed point from gauge/gravity duality, Phys. Rev. D 81 (2010) 046003 [arXiv:0912.4128] [INSPIRE].

[46] J. Alanen, K. Kajantie and K. Tuominen, Thermodynamics of quasi conformal theories from gauge/gravity duality, Phys. Rev. D 82 (2010) 055024 [arXiv:1003.5499] [InSPIRE].

[47] J. Alanen, T. Alho, K. Kajantie and K. Tuominen, Mass spectrum and thermodynamics of quasi-conformal gauge theories from gauge/gravity duality, Phys. Rev. D 84 (2011) 086007 [arXiv: 1107.3362] [INSPIRE].

[48] G. Bertoldi, F. Bigazzi, A. Cotrone and J.D. Edelstein, Holography and unquenched quark-gluon plasmas, Phys. Rev. D 76 (2007) 065007 [hep-th/0702225] [INSPIRE].

[49] C. Núñez, I. Papadimitriou and M. Piai, Walking dynamics from string duals, Int. J. Mod. Phys. A 25 (2010) 2837 [arXiv:0812.3655] [InSPIRE].

[50] S.P. Kumar, D. Mateos, A. Paredes and M. Piai, Towards holographic walking from $N=4$ super Yang-Mills, JHEP 05 (2011) 008 [arXiv:1012.4678] [INSPIRE].

[51] L. Anguelova, Electroweak symmetry breaking from gauge/gravity duality, Nucl. Phys. B 843 (2011) 429 [arXiv: 1006.3570] [INSPIRE].

[52] L. Anguelova, P. Suranyi and L. Wijewardhana, Holographic walking technicolor from D-branes, Nucl. Phys. B 852 (2011) 39 [arXiv:1105.4185] [INSPIRE].

[53] L. Anguelova, P. Suranyi and L.R. Wijewardhana, Scalar mesons in holographic walking technicolor, Nucl. Phys. B 862 (2012) 671 [arXiv:1203.1968] [INSPIRE].

[54] D. Kutasov, J. Lin and A. Parnachev, Conformal phase transitions at weak and strong coupling, Nucl. Phys. B 858 (2012) 155 [arXiv:1107.2324] [INSPIRE].

[55] D.K. Hong and H.-U. Yee, Holographic estimate of oblique corrections for technicolor, Phys. Rev. D 74 (2006) 015011 [hep-ph/0602177] [INSPIRE].

[56] K. Haba, S. Matsuzaki and K. Yamawaki, S parameter in the holographic walking/conformal technicolor, Prog. Theor. Phys. 120 (2008) 691 [arXiv:0804.3668] [INSPIRE]. 
[57] D.D. Dietrich and C. Kouvaris, Constraining vectors and axial-vectors in walking technicolour by a holographic principle, Phys. Rev. D 78 (2008) 055005 [arXiv:0805.1503] [INSPIRE].

[58] D.D. Dietrich and C. Kouvaris, Generalised bottom-up holography and walking technicolour, Phys. Rev. D 79 (2009) 075004 [arXiv:0809.1324] [InSPIRE].

[59] D.D. Dietrich, M. Jarvinen and C. Kouvaris, Mixing in the axial sector in bottom-up holography for walking technicolour, JHEP 07 (2010) 023 [arXiv:0908.4357] [INSPIRE].

[60] D. Kutasov, J. Lin and A. Parnachev, Holographic walking from tachyon DBI, Nucl. Phys. B 863 (2012) 361 [arXiv:1201.4123] [INSPIRE].

[61] C. Núñez, A. Paredes and A.V. Ramallo, Unquenched flavor in the gauge/gravity correspondence, Adv. High Energy Phys. 2010 (2010) 196714 [arXiv: 1002.1088] [INSPIRE].

[62] O. Bergman, S. Seki and J. Sonnenschein, Quark mass and condensate in HQCD, JHEP 12 (2007) 037 [arXiv: 0708.2839] [InSPIRE].

[63] A. Dhar and P. Nag, Sakai-Sugimoto model, tachyon condensation and chiral symmetry breaking, JHEP 01 (2008) 055 [arXiv:0708.3233] [INSPIRE].

[64] A. Dhar and P. Nag, Tachyon condensation and quark mass in modified Sakai-Sugimoto model, Phys. Rev. D 78 (2008) 066021 [arXiv: 0804.4807] [INSPIRE].

[65] N. Jokela, M. Järvinen and S. Nowling, Winding effects on brane/anti-brane pairs, JHEP 07 (2009) 085 [arXiv:0901.0281] [INSPIRE].

[66] D.B. Kaplan, J.-W. Lee, D.T. Son and M.A. Stephanov, Conformality lost, Phys. Rev. D 80 (2009) 125005 [arXiv:0905.4752] [INSPIRE].

[67] M. Järvinen and F. Sannino, Holographic conformal window - A bottom up approach, JHEP 05 (2010) 041 [arXiv: 0911.2462] [INSPIRE].

[68] O. Antipin and K. Tuominen, Constraints on conformal windows from holographic duals, Mod. Phys. Lett. A 26 (2011) 2227 [arXiv:0912.0674] [InSPIRE].

[69] R. Alvares, N. Evans and K.-Y. Kim, Holography of the conformal window, Phys. Rev. D 86 (2012) 026008 [arXiv: 1204.2474] [INSPIRE].

[70] D. Arean, I. Iatrakis, M. Järvinen and E. Kiritsis, V-QCD: spectra, the dilaton and the S-parameter, arXiv:1211.6125 [INSPIRE].

[71] K. Tuominen, Finite temperature phase diagrams of gauge theories, arXiv:1206.5772 [INSPIRE].

[72] J. Noronha, Polyakov loops in strongly-coupled plasmas with gravity duals, J. Phys. G 37 (2010) 094018 [arXiv: 1001.3155] [INSPIRE].

[73] M. Panero, Thermodynamics of the QCD plasma and the large-N limit, Phys. Rev. Lett. 103 (2009) 232001 [arXiv:0907.3719] [INSPIRE].

[74] J. Braun and H. Gies, Chiral phase boundary of QCD at finite temperature, JHEP 06 (2006) 024 [hep-ph/0602226] [INSPIRE].

[75] J. Braun and H. Gies, Scaling laws near the conformal window of many-flavor QCD, JHEP 05 (2010) 060 [arXiv: 0912.4168] [INSPIRE].

[76] M. Järvinen and F. Sannino, Extreme technicolor and the walking critical temperature, JHEP 02 (2011) 081 [arXiv: 1009.5380] [INSPIRE]. 
[77] J. Braun, C.S. Fischer and H. Gies, Beyond Miransky scaling, Phys. Rev. D 84 (2011) 034045 [arXiv: 1012 .4279] [INSPIRE].

[78] D.M. Broun, What lies beneath the dome?, Nature Phys. 4 (2008) 170.

[79] M. Teper, Large-N, PoS (LATTICE 2008) 022 [arXiv:0812.0085] [INSPIRE].

[80] K. Kajantie, M. Krssak, M. Vepsäläinen and A. Vuorinen, Frequency and wave number dependence of the shear correlator in strongly coupled hot Yang-Mills theory, Phys. Rev. D 84 (2011) 086004 [arXiv: 1104.5352] [INSPIRE]. 